\title{
Palimpsestfragmente der Homiliae I et III in nativitatem B. M. V. des Andreas von Kreta im Cod. Vind. theol. gr. 160*
}

\section{EINLEITUNG}

Bei der Erfassung des Cod. Vind. theol. gr. 160 - einer theologischen Sammelhandschrift (in ihrem ursprünglichen Bestand [f. $\left.5^{\mathrm{r}}-210^{\mathrm{v}}\right]$ mit den Homiliae in Hexaemeron des Basileios von Kaisareia und mit drei Werken des Gregorios von Nyssa, De opificio hominis, De beatitudinibus und De oratione dominica; alles in einer gepflegten kalligraphischen Minuskel in der Tradition der „Perlschrift“ aus der ersten Hälfte des 11. Jahrhunderts) - im Rahmen der Katalogisierung der griechischen Handschriften der Österreichischen Nationalbibliothek ${ }^{1}$ zeigte es sich, daß auf den sechs mit Fragmenten eines Evangelienlektionars ${ }^{2}$ gefüllten „Vor- und Nachsatzblättern“‘3 (f. $1^{\mathrm{r}}-4^{\mathrm{v}}$ und $\left.214^{\mathrm{r}}-215^{\mathrm{v}}\right)^{4}$ eine untere, um $90^{\circ}$ gedrehte, in zwei Kolumnen zu je 29 Zeilen angeordnete

\section{* Folgende Literatur wird grundsätzlich nur in Abkürzung zitiert:}

DE Strycker, Protevangelium = E. DE STRYCKer, Die griechischen Handschriften des Protevangeliums Iacobi, in: D. HARLFIngER (Hrsg.), Griechische Kodikologie und Textüberlieferung. Darmstadt 1980, 577-612.

EHRHARD, Überlieferung = A. EHRHARD, Überlieferung und Bestand der hagiographischen und homiletischen Literatur der griechischen Kirche von den Anfängen bis zum Ende des 16. Jahrhunderts. Erster Teil: Die Überlieferung I-III (Texte und Untersuchungen zur Geschichte der altchristlichen Literatur 50-52). Leipzig 1937-1952.

GeERARD, Clavis = M. GEerard, Clavis patrum graecorum I-V. Turnhout 1974-1987.

GEERARD-Noret, Clavis Suppl. = M. GEERARD-J. NorET, Clavis patrum graecorum. Supplementum. Turnhout 1998.

KotTER, Die Schriften des Johannes von Damaskos V = Die Schriften des Johannes von Damaskos ... V. Opera homiletica et hagiographica, besorgt von B. KotTER (Patristische Texte und Studien 29). Berlin-New York 1988.

${ }^{1}$ H. Hunger-O. KResten-Chr. HAnnick, Katalog der griechischen Handschriften der Österreichischen Nationalbibliothek III/2. Codices theologici 101-200 (Museion N. F. IV/1, 3, 2). Wien 1984, 242-247.

${ }^{2}$ Lesungen für die Zeit von Ostermontag (bzw. Ostersonntag; s. gleich im folgenden) bis Freitag nach Ostern; vgl. die Angaben bei Hunger-Kresten-HannicK, Katalog 242-244 (zu Nr. 1 und 7); die am zu rekonstruierenden Beginn der Fragmente (f. 214r; vgl. unten,

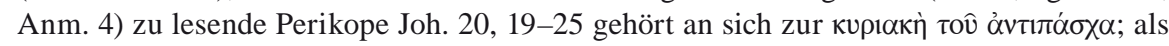
Lesung zum Ostersonntag (d. h. vor der auf f. 214 $-1^{\mathrm{r}}$ eingetragenen, dem Ostermontag

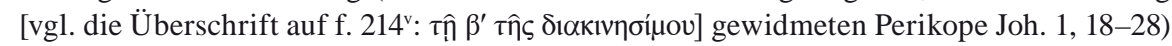
wäre Joh. 1, 1-17 vorzusehen; so zumindest die Hinweise bei C. R. GrEGORY, Textkritik des Neuen Testaments I. Leipzig 1900, 344-345; vgl. aber J. MATEOS, Le Typicon de la Grande Église ... II. Le cycle des fêtes mobiles (Orientalia Christiana Analecta 166). Roma 1963,

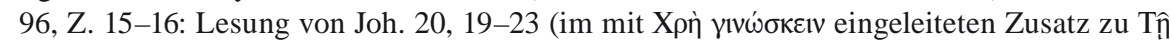

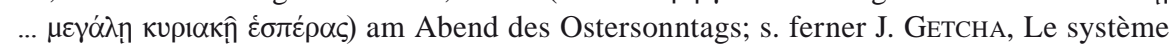
des lectures bibliques du rite byzantin, in: A. M. TriacCA-A. Pistoia (Hrsg.), La liturgie, interprète de l'Écriture I. Les lectures bibliques pour les dimanches et fêtes. Conférences Saint-Serge. XLVIII ${ }^{e}$ Semaine d'études liturgiques, Paris, 25-28 juin 2001 (Bibliotheca «Ephemerides liturgicae». Subsidia 119). Roma 2002, 40. - Die im Katalog des Jahres 1984 vorgenommene (und von K. Aland-M. Welte-B. Köster-K. JunacK, Kurzgefaßte Liste der griechischen Handschriften des Neuen Testaments. Zweite, neubearbeitete und ergänzte Auflage [Arbeiten zur neutestamentlichen Textforschung 1]. Berlin-New York 1994, 240, Nr. 1347, übernommene) Datierung der Schrift der Lektionarfragmente in die erste Hälfte 
Schrift zu erkennen war ${ }^{5}$, die seinerzeit als „rechtsgeneigte ogivale Majuskel“ beschrieben und summarisch dem 9. Jahrhundert zugewiesen wurde ${ }^{6}$. Aus dem Verhältnis der scriptura inferior zur scriptura superior folgt, daß hier drei Blätter eines alten Codex zwecks Wiederverwertung palimpsestiert und zu drei Doppelblättern gefaltet worden waren, woraus sich für die „Mutterhandschrift“ der palimpsestierten Folien ein Format von (са.) $350 \times 235$ mm ergibt.

Hinsichtlich des Inhalts der scriptura inferior scheiterten bei der seinerzeitigen Katalogisierung alle Identifizierungsversuche: Es waren zwar damals unter Ultraviolettlicht einige Worte zu lesen, die aufgrund ihres eindeutig „fachspezifischen“ (d. h. theologischen) Charakters eine vielversprechende Ausgangsbasis für eine entsprechende Suche in den in den siebziger Jahren des 20. Jahrhunderts zur Verfügung stehenden lexikalischen Hilfsmitteln ${ }^{7} \mathrm{zu}$ bieten schienen ${ }^{8}$, doch führten alle damaligen Bemühungen zu keinem Erfolg. Einzig und allein der Umstand, daß die Fragmente mehrmals in dezidierter Weise Maria als $\theta \varepsilon$ có́кo ansprachen $^{9}$, legte die Annahme nahe, daß hier ein („orthodoxer“) mariologischer Text (mit einiger Wahrscheinlichkeit frühestens aus der Zeit der Konzilien von Ephesos und Chalkedon) vorliegen müsse.

Als dann im Rahmen des Forschungsprojekts «Rinascimento virtuale» des Programms “Culture 2000” der Europäischen Union ${ }^{10}$ eine kurz zuvor in Italien entwickelte fortschrittliche Technologie der Palimpsestphotographie eingesetzt

des 14. Jahrhunderts (vgl. Hunger-Kresten-Hannick, Katalog 242 [im „Vorspann“]) läßt sich heute nicht mehr aufrechterhalten (s. dazu auch unten, S. 47-48).

3 Der Begriff „Vor- und Nachsatzblätter“ wurde hier deswegen unter Anführungszeichen gesetzt, weil sich die Funktion dieser Folien in den verschiedenen Stadien ihrer Geschichte zweimal geändert hat; vgl. dazu unten, S. 47-51.

${ }^{4}$ Die korrekte Blattabfolge im ursprünglichen Zustand des Lektionars lautet übrigens $\mathrm{f}$. $214^{\mathrm{r}-\mathrm{v}}$. $1^{\mathrm{r}}-4^{\mathrm{v}}$. 215 $5^{\mathrm{r}-\mathrm{v}}$ (s. dazu auch unten, S. 48-49).

${ }^{5}$ Die untere Schrift der ebenfalls palimpsestierten (und ebenfalls als „Nachsatzblätter“ zu wertenden) Folien $213^{r-v}$ und $216^{r-v}$ des Cod. Vind. theol. gr. 160 ist nicht Gegenstand des vorliegenden Beitrags, da diese Blätter einem anderen Codex entnommen wurden als die Folien $1^{\mathrm{r}}-4^{\mathrm{v}}$ und $214^{\mathrm{r}}-215^{\mathrm{v}}$; man vergleiche dazu die Angaben bei HungER-KRESTEN-HANNICK, Katalog (wie in Anm. 1) 244 (Nr. 6) und 246 (Schreiber g) (zur Datierung dieser anspruchslosen Minuskel - 12. Jahrhundert - vgl. a. O. 242 [Vorspann]).

${ }^{6}$ Hunger-Kresten-Hannick, Katalog (wie in Anm. 1) 242 und 246. - Bei einem nunmehr (nach dem Vorliegen der entsprechenden Palimpsestphotographien) auf einer ausreichenden Basis möglichen paläographischen Vergleich mit der seinerzeit zitierten Tav. 12 bei G. CAVALLO, Funzione e struttura della maiuscola greca tra i secoli VIII-XI, in: La Paléographie grecque et byzantine. Paris, 21-25 octobre 1974 (Colloques Internationaux du Centre National de la Recherche Scientifique 559). Paris 1977, 118, fällt auf, daß die Rechtsneigung in der palimpsestierten Majuskel der Folien $1^{\mathrm{r}}-4^{\mathrm{v}}$ und $214^{\mathrm{r}}-215^{\mathrm{v}}$ des Cod. Vind. theol. gr. 160 etwas geringer ist als im von Cavallo abgebildeten Cod. Par. gr. 923 (vgl. das dem vorliegenden Beitrag beigegebene Tafelmaterial [Abb. 1-6]; vgl. auch die ausführliche Diskussion der Schrift unserer Fragmente unten, S. 45-46).

Z. B. bei G. W. H. LAMPE, A Patristic Greek Lexicon. Oxford 1961.

8 Z. B. (in zum Teil „normalisierter“ Transkription) $\alpha \rho ı \tau \tau o \tau \varepsilon ́ \chi v \alpha \varsigma ~\left(F r a g m e n t ~ A^{r}\left[=\right.\right.$ f. $\left.3^{r}+2^{v}\right]$, Kolumne I, Z. 2; im folgenden unter Aufnahme der Zählung in der beigegebenen Transkription bzw. Edition als $\mathrm{A}^{\mathrm{r}} \mathrm{I}^{2}$ bezeichnet; vgl. dazu auch unten, S. 9 [mit Anm. 22]),

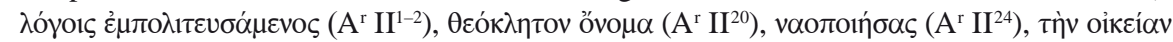

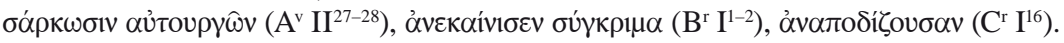

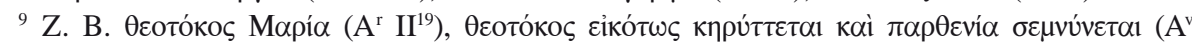
$\left.\mathrm{I}^{17-19}\right)$.

${ }^{10}$ Dieses (mit dem deutschen Paralleltitel „Digitale Palimpsestforschung“ versehene) internationale Projekt wurde in den Jahren 2001-2004 unter der Leitung von Dieter Harlfinger (Universität Hamburg) durchgeführt; von österreichischer Seite beteiligten sich die (nunmehr in ein Institut für Byzanzforschung umgewandelte) Kommission für Byzantinistik 
werden konnte, wurden bei der Auswahl der innerhalb des Projekts zu untersuchenden griechischen Palimpseste an der Österreichischen Nationalbibliothek Wien ${ }^{11}$ auch die genannten sechs Folien aus dem Cod. Vind. theol. gr. 160 berücksichtigt und im Rahmen einer im Januar 2003 von der Firma Fotoscientifica Parma (D. Broia) in Wien durchgeführten Photokampagne aufgenommen; besonders vorteilhaft erwies sich dabei der Umstand, daß die palimpsestierten Blätter im Zuge einer Teilrestaurierung dem Cod. Vind. theol. gr. 160 entnommen wurden, wodurch es möglich war, diese Folien plan zu photographieren und damit auch die bisher durch die Bindung der Handschrift ${ }^{12}$ kaum oder nicht lesbaren Zeilen des unteren Textes zu erfassen.

Nach dem Abschluß der Photokampagne standen somit sechs vorzügliche, großformatige Farbphotographien zur Verfügung, auf denen die ursprüngliche Schrift der drei zu untersuchenden „originalen“ Blätter weitgehend (allerdings nicht vollständig ${ }^{13}$ ) zu lesen war $^{14}$. Allein - auch bei dieser nunmehr geradezu idealen, zu einer ersten Transkription führenden Ausgangsbasis erwies es sich als unmöglich, den palimpsestierten Text mit Hilfe der Datenbank des „Thesaurus Linguae Graecae“ (TLG) zu identifizieren. Auch die Tatsache, daß in einer Passage ( $\mathrm{C}^{\mathrm{v}} \mathrm{I}^{12-29}$ ) ein umfangreicheres Zitat aus Num. 36, 7-8 festgestellt werden konnte, ergab fürs erste keine konkrete Handhabe für eine nähere Bestimmung des ursprünglichen Inhalts der zu bearbeitenden Folien.

Bei einer wiederholten Lektüre des transkribierten Textes mußte freilich der ursprüngliche Gedanke aufgegeben werden, der die Fragmente mit einem theologischen Traktat aus der Zeit der Auseinandersetzungen zwischen Monophysitismus, Dyophysitismus und „chalkedonensischer“ Formulierung zu

der Österreichischen Akademie der Wissenschaften und die Handschriften-, Autographenund Nachlaß-Sammlung der Österreichischen Nationalbibliothek.

${ }^{11}$ Vgl. dazu E. GAmillscheg, Rinascimento Virtuale - Digitale Palimpsestforschung. ÖNB Newsletter 1 (März 2003) 10.

12 Typischer Einband der Bibliotheca Caesarea Vindobonensis, im Jahre 1755 unter dem damaligen Präfekten der Hofbibliothek, Gerard van Swieten, angelegt; vgl. H. HungER, Katalog der griechischen Handschriften der Österreichischen Nationalbibliothek I. Codices historici. Codices philosophici et philologici (Museion N. F. IV/1, 1). Wien 1961, XVI.

${ }^{13}$ An allen Stellen, an denen Teile der oberen Schrift Teile der unteren Schrift bedecken bzw. an denen so gut wie keine Reste der unteren Schrift erkennbar sind, scheiterte auch die Technik der uns zur Verfügung stehenden multispektralen Photographie. - Bei allen jenen Passagen, bei denen die Photographien keine ausreichende Ausgangsbasis für eine korrekte Lesung boten, wurde der Text am Original unter Ultraviolettlicht einer nochmaligen genauen Überprüfung unterzogen. Diese Autopsie war auch an allen jenen Stellen notwendig, wo die digitale Nachbearbeitung der Aufnahmen aufgrund ambivalenter Details (etwa Schmutzspuren, die Buchstabenresten ähneln) im „Endprodukt“ zu einer irreführenden Rekonstruktion einzelner Buchstabenformen geführt hatte; vgl. etwa Fragment $\mathrm{C}^{\mathrm{v}} \mathrm{I}^{26-27}$, wo zwei aufeinanderfolgende Iota in den Worten $\mathrm{Y} \mid \mathrm{IOI} \overline{\mathrm{IH} \Lambda}$ in der digitalen Nachbearbeitung zu einem Ny „umgestaltet“ worden waren. Ein zusätzliches Hilfsmittel bei dieser Überprüfung stellten die von der Firma Fotoscientifica angefertigten und uns während der letzten Arbeitsphase zur Verfügung gestellten Ultraviolett-Rohdateien dar, die den „Urzustand“ der photographischen Aufnahmen (d. h. vor der digitalen Nachbearbeitung) dokumentieren.

${ }^{14}$ Vgl. die dem vorliegenden Beitrag beigegebenen Photographien, welche die originalen Blattausmaße um ca. 50\% verkleinern. - An allen jenen Stellen, an denen die von uns publizierten Aufnahmen (eben aufgrund der in der vorangehenden Anmerkung apostrophierten digitalen Nachbearbeitung) prima vista einen anderen (von unserer Transkription abweichenden) Buchstabenbestand zu ergeben scheinen, erbitten die Autoren des vorliegenden Beitrags einen Vertrauensvorschuß: Alle diese scheinbar „abweichenden“ Lesungen basieren auf einer sorgfältigen mehrmaligen Überprüfung entweder des Originals oder der Ultraviolett-Rohdateien. 
den Naturen Christi, also grob gesprochen aus der Zeit der ersten Hälfte des 5. Jahrhunderts, in Verbindung bringen wollte: Der auf einem hohen sprachlichen Niveau stehende Text wies vielmehr Eigenheiten auf, die auf einen homiletischen Charakter der Fragmente hindeuteten, wobei sich stilistische Reminiszenzen an die Homilia in transfigurationem Domini des Andreas von Kreta einstellten ${ }^{15}$. Als es sich dann zeigte, daß das homiletische Euvre des Andreas vom TLG noch nicht erfaßt worden war $^{16}$ und daß im „Index methodicus“ bei F. Cavallera s. v. „De beata virgine Maria et eius festis“ Andreas im Unterabschnitt „In Nativitatem“ an prominenter Stelle aufscheint ${ }^{17}$, war der entscheidende Schritt zu einer Identifizierung des Inhalts der unteren Schrift

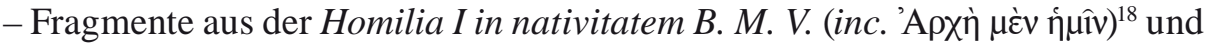

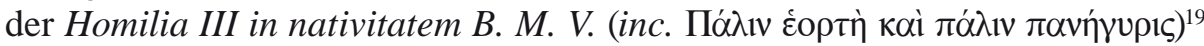
des Andreas von Kreta ${ }^{20}$ - der eingangs genannten Palimpsestblätter im Cod. Vind. theol. gr. 160 getan.

${ }^{15}$ D. h. an einen Text, mit dem einer der beiden Autoren der vorliegenden Studie im Zusammenhang mit der Identifizierung der patristischen Zitate im hesychastischen Tomos von 1341 ad nauseam zu tun hatte: O. KResten, Studien zum Tomos des Jahres 1341, in: H. Hunger-O. Kresten, Studien zum Patriarchatsregister von Konstantinopel II (Sitzungsber. phil.-hist. Kl. Österr. Akad. Wiss. 647). Wien 1997, 98-106. - Als weitere „Assoziationshilfe“ dienten die Erinnerungen an ein höchst instruktives Referat, das Diether Roderich Reinsch im Jahre 1998 in Cremona auf dem V. Internationalen Kongreß für griechische Paläographie gehalten hatte: D. R. REINSCH, Literarische Bildung in Konstantinopel im 7. und 8. Jahrhundert: Das Zeugnis der Homiletik, in: I manoscritti greci tra riflessione e dibattito. Atti del V Colloquio Internazionale di Paleografia Greca (Cremona, 4-10 ottobre 1998), a cura di G. PrATO, Bd. I (Papyrologica Florentina XXXI). Firenze 2000, 29-46. Als sich die in die Richtung „Andreas von Kreta“ gehende Vermutung als richtig erwies, stellten sich auch Erinnerungen an den von Theocharis Detorakis untersuchten Wortschatz des Andreas ein: Th. Detorakis, Le vocabulaire d’André de Crète. Mots non thésaurisés par G. W. H. Lampe. Jahrbuch der Österreichischen Byzantinistik 36 (1986) 45-60 (wobei der Untertitel gleichzeitig eine Erklärung dafür gab, daß die seinerzeit, im Zuge der Katalogisierung der Wiener Codices theologici graeci, unternommenen Identifizierungsversuche auf der Basis der damals zugänglichen lexikalischen Hilfsmittel [vgl. Anm. 7-8] erfolglos bleiben mußten).

16 Auch zum Zeitpunkt des Abschlusses des vorliegenden Beitrags (Juni 2008) ist in der Online-Version des TLG lediglich der Wortschatz von zwei Kanones des Andreas berücksichtigt.

${ }^{17}$ F. Cavallera, Patrologiae cursus completus, accurante J.-P. Migne. Series graeca, Indices. (Ndr.) Turnhout s. a., 166.

18 Vgl. GeErard, Clavis III 542 (Nr. 8170); PG 97, 805-820.

19 Geerard, a. O. 542 (Nr. 8172); PG 97, 844-861; diese Homilie wird bisweilen auch unter dem Namen des Ioannes von Damaskos tradiert (wohl eine jener Zuweisungen in der Überlieferung, die auf ein nicht richtig interpretiertes oder in irrtümlicher Weise automa-

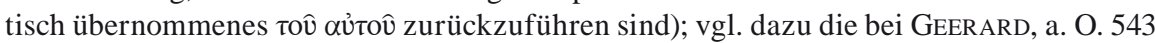
(„nota“ nach Nr. 8173), angegebene Literatur bzw. R. LAURENTIN, Court traité de theólogie mariale. Paris 1954, 171, und D. CASAGRANDE, Enchiridion marianum biblicum patristicum. Rom 1974, 1453, Anm. 1.

${ }_{20} \mathrm{Zu}$ den Homiliae in nativitatem B. M. V. des Andreas von Kreta vgl. (über die bibliographischen Angaben bei GeErard, Clavis III 542f., und bei GeErard-Noret, Clavis Suppl.

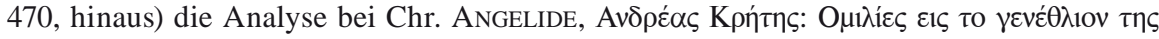

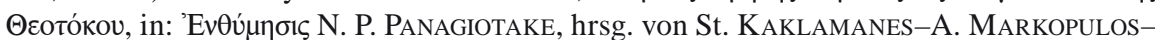
G. Mauromates. Herakleion 2000, 1-11; für die vorliegende Untersuchung von geringem Wert ist die (auf der Grundlage des Textes in der „Patrologia graeca“ angefertigte) italienische Übersetzung der Marienhomilien des Andreas von Kreta: Andrea di Creta, Omelie mariane. Traduzione, introduzione e note a cura di V. FAzzo (Collana di Testi patristici 63). Roma 1987. - Die Ausführungen bei J. E. BICKERSTETH, Unedited Greek Homilies (acephalous, anonymous or attributed to John Chrysostom) for Festivals of the Virgin Mary. Orientalia Christiana Periodica 46 (1980) 474-480, geben für die im folgenden in unserem 
Konkreter konnten dabei folgende Textpassagen identifiziert werden:

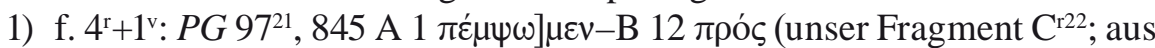
Homilia III);

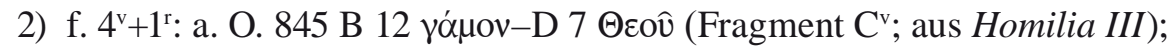

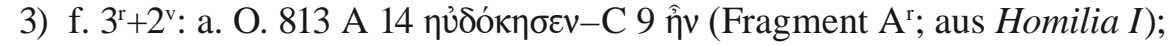

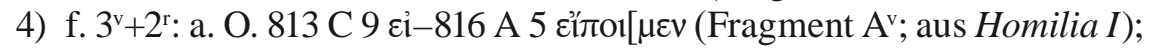

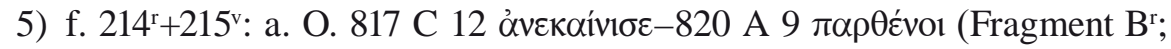
aus Homilia I);

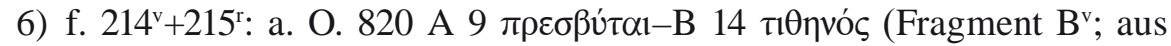
Homilia I).

Unter der gleich im folgenden an Hand kodikologischer Details zu untermauernden Voraussetzung, daß die Homilia III im originalen Codex nach, nicht vor Homilia I kopiert worden war $^{23}$, läßt sich die Blattabfolge im ursprünglichen Codex - einem Homiliar, wie mit hoher Wahrscheinlichkeit vermutet werden darf - wie folgt wiederherstellen:

1) f. $3^{\mathrm{r}}$ (inc. mut.) $+2^{\mathrm{v}}$ und f. $3^{\mathrm{v}}+2^{\mathrm{r}}$ (des. mut.) (f. $\left.\mathrm{A}^{\mathrm{r}-\mathrm{v}}\right)$;

2) f. $214^{\mathrm{r}}$ (inc. mut.) $+215^{\mathrm{v}}$ und f. $214^{\mathrm{v}}+215^{\mathrm{r}}$ (des. mut.) (f. $\left.\mathrm{B}^{\mathrm{r}-\mathrm{v}}\right)$;

3) f. $4^{\mathrm{r}}$ (inc. mut.) $+1^{\mathrm{v}}$ und f. $4^{\mathrm{v}}+1^{\mathrm{r}}$ (des. mut.) (f. $\mathrm{C}^{\mathrm{r}-\mathrm{v}}$ ).

Bei dem Versuch einer korrekten Rekonstruktion des kodikologischen Aufbaus der ursprünglichen Handschrift, der diese drei Folien entstammen, kommt einem die Tatsache zu Hilfe, daß auf f. $A^{r}$ (d. h. heute auf f. $3^{r}$ im äußeren Freirand nach der Wiederverwendung des Blattes, etwa in Höhe der 3. Z. v. u. der scriptura posterior), und zwar rechts im oberen Freirand des ursprünglichen Blattes, eine (von erster Hand angebrachte) Kustode Z (darüber ein Zahlstrich, darunter ein Zierstrich) sichtbar ist ${ }^{24}$; analog dazu findet sich auf f. $\mathrm{C}^{\mathrm{v}}$ links unten (d. h. heute auf f. $1^{\mathrm{r}}$ am rechten Ende der 3. Z. v. u. der scriptura posterior) (ebenfalls von erster Hand) die korrespondierende Kustode $\mathrm{Z}$ (darüber ein Zahlstrich; ein Zierstrich unter der Kustode wurde offensichtlich nicht angebracht ${ }^{25}$; wir haben hier also die beiden äußeren Blätter der 7. Lage des originalen Homiliars vor uns ${ }^{26}$; für die seinerzeitige Zusammengehörigkeit

Beitrag diskutierten Fragen nichts her. - Nachdrücklich sei darauf hingewiesen, daß die (von uns aus Gründen des „bequemeren“ Zitierens beibehaltene) Zählung der genannten Marienhomilien des Andreas von Kreta als „I“ und „III“ ausschließlich eine künstliche, in dieser Form erst im Druck in Mignes „Patrologia graeca“ eingeführte Numerierung ist, die als solche keinen Rückhalt in der handschriftlichen Tradition hat (nur im Cod. Athen. Ethn. Bibl. 2734 [vgl. unten, S. 19f. mit Anm. 96] läßt sich eine direkte Abfolge der ersten, zweiten und dritten Marienhomilie des Andreas von Kreta feststellen).

${ }^{21}$ Die Andreas-Edition in Mignes „Patrologia graeca“ ist keine ideale Ausgangsbasis für textkritische Untersuchungen, da sie nichts anderes als einen (bisweilen über mehrere Zwischenstationen gehenden) Abdruck verschiedener älterer Ausgaben (vgl. dazu unten, S. 36-38) bietet; angesichts der allgemeinen Verbreitung der „Patrologia“ erweist sich freilich eine erste Textidentifikation auf ihrer Basis als die gangbarste Lösung.

${ }^{22}$ Unsere Zählung der Fragmente entspricht bereits der rekonstruierten originalen Blattabfolge im ursprünglichen Codex (vgl. gleich im folgenden; s. auch schon den kurzen Hinweis oben, Anm. 8).

${ }^{23}$ Zur Überlieferungsgemeinschaft dieser beiden Homilien s. auch unten, S. 12ff.

${ }^{24}$ Vgl. dazu die beigegebene Abb. 1. - Entfernt vergleichbar ist etwa die Gestaltung der Kustode KE auf f. $191^{\text {r }}$ des Cod. Par. gr. 2389: vgl. Taf. IX bei H. OMONT, Fac-similés des plus anciens manuscrits grecs en onciale et en minuscule de la Bibliothèque Nationale du IV ${ }^{\mathfrak{e}}$ au XII siècle. Paris 1892.

${ }^{25}$ Vgl. dazu die beigegebene Abb. 6.

${ }^{26}$ Die Anbringung von Kustoden auf dem ersten Recto und auf dem letzten Verso einer Lage ist für das 9. Jahrhundert zwar auffällig, aber keineswegs ausgeschlossen; vgl. etwa den 
dieser Folien sprechen auch die erkennbaren, in Richtung auf ein ehemaliges Bifolium zu interpretierenden Reste des originalen Liniensystems ${ }^{27}$ auf dem heutigen f. $3^{\mathrm{v}}+2^{\mathrm{r}}$ (f. $\mathrm{A}^{\mathrm{v}}$ ) in Korrespondenz zu f. $4^{\mathrm{r}+1^{\mathrm{v}}}$ (f. $\left.\mathrm{C}^{\mathrm{r}}\right)^{28}$.

Daraus folgt, daß sich in dem ursprünglichen Homiliar vor unserem $\mathrm{f}$. $\mathrm{A}^{\mathrm{r}}$ sechs weitere Lagen befunden haben müssen; mit ihnen (d. h. mit dem Ende der 6. Lage) ging auch der Beginn der Homilia I in nativitatem B. M. V., und

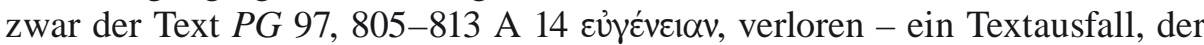
rund 160 Zeilen im Druck bei Migne entspricht. Setzt man diese Beobachtung mit dem Faktum in Verbindung, daß auf f. $\mathrm{A}^{\mathrm{r}-\mathrm{v}}$ etwa 46 Migne-Zeilen, auf f. $\mathrm{B}^{\mathrm{r}-\mathrm{v}}$ etwa 42 Migne-Zeilen und auf f. $\mathrm{C}^{\mathrm{r}-\mathrm{v}}$ etwa 47 Migne-Zeilen enthalten sind, ist der Schluß erlaubt, daß die Homilia I in nativitatem B. M. V. ungefähr auf dem 5. Blatt dieser 6. Lage eingesetzt haben muß ${ }^{29}$.

Die Homilia I in nativitatem B. M. V. bricht, wie bereits ausgeführt, auf f. Av

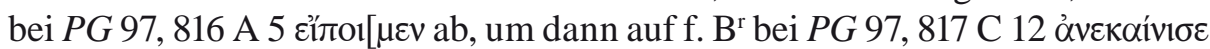
ihre Fortsetzung zu finden; dazwischen fehlen rund 90 Migne-Zeilen ${ }^{30}$, wo-

ersten Teil des Cod. Sinait. gr. 210 (+ Sinait. gr. NE Meg. Perg. 12) aus dem Jahre 861/862 (Beschreibung bei D. Harlfinger-D. R. ReinsCh-J. A. M. SonderkamP-G. Prato, Specimina Sinaitica. Die datierten griechischen Handschriften des Katharinenklosters auf dem Berge Sinai, 9.-12. Jahrhundert. Berlin 1983, 13; Abb. einer auf f. 41 $1^{\mathrm{r}}$ angebrachten Kustode auf Taf. 2); vgl. ferner die Hinweise bei B. Mondrain, Les signatures des cahiers dans les manuscrits grecs, in: Recherches de codicologie comparée. La composition du codex au Moyen Âge, en Orient et en Occident. Textes édités par Ph. Hoffmann. Paris 1998, 21-48; ungewöhnlich ist bei unserem Fragment nur die Stellung der das Ende der Lage markierenden Kustode in der äußeren unteren Ecke des letzten Verso (Typ Nr. 8 bei MondRain 32 [nur mit relativ späten Beispielen]). - In dem von uns zum Vergleich herangezogenen Cod. Par. gr. 2389 (vgl. Anm. 24) stammen die Kustoden auf dem jeweiligen letzten Lagenverso nicht von erster Hand (freundlicher Hinweis von Christian Foerstel).

${ }^{27}$ Es entspricht im großen und ganzen dem Typ 21A2a Leroy-Sautel (Répertoire de réglures dans les manuscrits grecs sur parchemin. Base de données établie par J.-H. SAUTEL à l'aide du fichier Leroy et des catalogues récents [Bibliologia 13]. Turnhout 1995, 46); die zusätzliche untere (mit Bezug auf die scriptura inferior) Linie (vgl. Typ 22C2 Leroy-Sautel) dürfte erst sekundär, d. h. als Begrenzung des Schriftspiegels der scriptura superior (rechts im Falle von Recto-Seiten, links im Falle von Verso-Seiten des Lektionars) angebracht worden sein; offensichtlich weitere sekundäre „Nachbesserungen“ des originalen Liniensystems anläßlich der Wiederverwendung der Blätter nach ihrer Palimpsestierung erschweren einwandfreie Aussagen zur Linierung des ursprünglichen Homiliars.

${ }^{28}$ Innere Seiten des äußeren Doppelblattes dieser originalen Lage: Haarseiten (d. h. daß die Seiten $\mathrm{A}^{\mathrm{r}}$ und $\mathrm{C}^{\mathrm{v}}$ als Fleischseiten die lex Gregory einhalten; s. gleich im folgenden).

${ }^{29}$ Spekulationen über den Inhalt des alten Homiliars vor dem 5. Blatt der 6. Lage sind müßig, da über den Charakter dieser Sammlung (auch unter Heranziehung der Parallelüberlieferung der Homiliae in nativitatem B. M. V. des Andreas von Kreta; vgl. dazu auch gleich im folgenden) keine wirklich konkreten Aussagen gemacht werden können: Das Fest Mariä Geburt fällt auf den 8. September; die Bandbreite der in den vormetaphrastischen Jahrespanegyrika (bzw. Halbjahrespanegyrika) davor enthaltenen Texte ist nicht gering; vgl. etwa EHRHARD, Überlieferung I 154ff. (,Die alten Jahressammlungen“) und II 3ff. („Die Jahrespanegyriken“); für den 8. September kämen unter anderem auch das Protevangelium Iacobi (BHG 1046; vgl. dazu DE STRYCKeR, Protevangelium 577-612) oder die pseudodamaszenische Oratio in nativitatem sanctae Dei genitricis Mariae (KoTTER, Die Schriften des Johannes von Damaskos V 147-182; zur gelegentlichen Zuschreibung der Homilia III in nativitatem B. M. V. des Andreas von Kreta an Ioannes von Damaskos vgl. schon oben, Anm. 19) in Frage (zu weiteren zum 8. September tradierten Homilien vgl. ohne Anspruch auf Vollständigkeit - die Angaben zu den weiter unten [S. 13ff.] diskutierten Textzeugen).

${ }^{30}$ Die Autoren des vorliegenden Beitrags sind sich der Tatsache bewußt, daß der (faute de mieux unternommene) Rekurs auf Migne-Zeilen zum Zwecke der Rekonstruktion des Umfangs von Textverlusten bzw. der ursprünglichen Lagenverhältnisse kein idealer Ausgangspunkt für derartige Rechenoperationen ist, da diese Basis mit mehreren Unsicherheitsfak- 
durch sich nach den soeben festgehaltenen Werten zwischen f. A und f. B mit hoher Wahrscheinlichkeit ein Ausfall von zwei Blättern des ursprünglichen Homiliars ergibt. Demzufolge ist unser f. B das 4. Blatt der 7. Lage des einer späteren Palimpsestierung unterworfenen Codex. Diese Beobachtung läßt sich zusätzlich durch einen Verweis auf die lex Gregory absichern - $\mathrm{f}$. $\mathrm{A}^{\mathrm{r}}$ ist, wie es sich für einen „korrekten“ Lagenbeginn ziemt, eine Fleischseite, f. $\mathrm{B}^{\mathrm{r}}$ hingegen eine Haarseite ${ }^{31}$. Der Text der Homilia I in nativitatem B. $M$. V. endet auf f. B ${ }^{\mathrm{v}}$

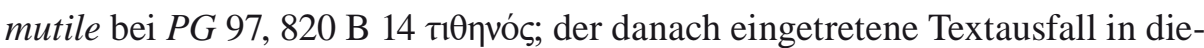
ser Homilie beläuft sich auf 14 Migne-Zeilen.

Fürs erste nicht in gleicher Weise sicher ist das Ergebnis der Überlegungen zum Umfang und zum Inhalt des Textverlustes zwischen $\mathrm{f}$. $\mathrm{B}^{\mathrm{v}}$ und $\mathrm{f}$. $\mathrm{C}^{\mathrm{r}}$ : Dieses letzte Blatt der 7. Lage beginnt mutile in der Homilia III in nativitatem B. M. V. des Andreas von Kreta bei PG 97, 845 A $1 \pi \varepsilon \dot{\mu} \mu \omega] \mu \varepsilon v$; es fehlt davor auf jeden Fall der Beginn dieser Homilie im Umfang von ca. 32 Migne-Zeilen ${ }^{32}$. Geht man in Form einer Arbeitshypothese von der Annahme aus, daß im ursprünglichen Homiliar die Homilia III direkt auf die Homilia I folgte, so ergäbe das bei einem Ausfall von 14 (Ende der Homilia I) + 32 (Beginn von Homilia III) = 46 Migne-Zeilen zwischen $\mathrm{f}$. B und $\mathrm{f}$. C ein Blatt des alten Codex für diese 7. Lage, die sich somit wie folgt (mit den entsprechenden Hinweisen auf den Wechsel von Fleisch- und Haarseiten) als Ternio rekonstruieren ließe:

\begin{tabular}{l|l|c|c|c|c|}
\hline \\
2 \\
3 \\
4 \\
5 \\
6
\end{tabular}

Ein derartiger Lagenumfang ist zwar für Pergamentcodices des 9. Jahrhunderts keineswegs auszuschließen, aber trotzdem auffällig; man würde eher einen Quaternio erwarten ${ }^{33}$, der sich - unter der Voraussetzung, daß zwischen den Fragmenten B und C nicht ein Blatt in Verlust geriet, sondern drei Blätter ausgefallen sind - in folgender Rekonstruktion (wiederum mit den entsprechenden Hinweisen auf den Wechsel von Fleisch- und Haarseiten) graphisch darstellen ließe:

toren belastet ist (man denke nur an die Kürzungsmöglichkeiten bei nomina sacra, die bei Migne natürlich stets ausgeschrieben werden), die das Resultat verfälschen könnten.

31 Die hier beschriebene Verteilung von Fleisch- und Haarseiten trifft sowohl für eine Rekonstruktion der 7. Lage auf der Basis eines Quaternio als auch für einen Rekonstruktionsversuch über die Annahme eines Ternio-Umfangs für diese Lage zu, kann also bei der Entscheidung der Frage, ob die 7. Lage ursprünglich acht (oder am Ende gar zehn) oder nur sechs Blätter enthielt, nicht als Argument herangezogen werden (vgl. im folgenden).

32 Und zwar unter Berücksichtigung der (mit einem Wert von \pm 4 Migne-Zeilen berechneten) Überschrift $P G$ 97, 844 B 7-12.

${ }_{33} \mathrm{Zu}$ den gebräuchlichen Lagenzusammensetzungen in byzantinischen Codices vgl. die Ausführungen bei J. IRIGoIN, Les cahiers des manuscrits grecs, in: Recherches de codicologie comparée (wie in Anm. 26) 1-19; instruktiv auch (wenngleich ausschließlich auf „lateinischem“ Material aufbauend) L. GILISSEN, La composition des cahiers. Le pliage du parchemin et l'imposition. Scriptorium 26 (1972) 3-33. - Nur en passant: Die von unseren Palimpsestfragmenten paläographisch nicht weit entfernten Codd. Par. gr. 437 und Par. gr. 2389 (vgl. die Abbildungen bei OMONT, Fac-similés [wie in Anm. 24], Taf. XIV und IX) setzen sich aus regelmäßigen Quaternionen zusammen (freundliche Auskunft von Christian Foerstel). 


\begin{tabular}{|l}
\hline 1 \\
2 \\
4 \\
5 \\
6 \\
7 \\
8
\end{tabular}

Eine einigermaßen zuverlässige Entscheidung, welche der beiden hier rekonstruierten Möglichkeiten der Zusammensetzung der 7. Lage des alten Homiliars, dem die Wiener Palimpsestfragmente entnommen worden waren, zutrifft $^{34}$, ließe sich nur auf dem Wege einer eingehenden Erörterung der sonstigen handschriftlichen Bezeugung der beiden genannten Marienhomilien des Andreas von Kreta treffen. Zu diesem Zwecke wurde - ohne den geringsten Anspruch auf Vollständigkeit - auf der Basis der ausgedehnten Untersuchungen Albert Ehrhards zur Überlieferung der homiletischen und hagiographischen Literatur der griechischen Kirche ${ }^{35}$ bzw. mit Hilfe der Diplomarbeit von Pauline D. Eyre zum homiletischen Euvre des Andreas von Kreta ${ }^{36}$ und der Handschriftenlisten von Robert E. Sinkewicz ${ }^{37}$ die folgende Übersicht zu Andreas-Codices erstellt ${ }^{38}$, wobei das Hauptaugenmerk auf den ältesten Überlieferungsträgern bzw. auf jenen Handschriften lag, welche die Homilia I und die Homilia III in nativitatem B. M. V. des Andreas von Kreta in Überlieferungsgemeinschaft enthalten ${ }^{39}$ :

${ }^{34}$ Zur theoretisch ebenfalls denkbaren Möglichkeit, daß die Lage jenes alten Homiliars, dem unsere Andreas-Fragmente entstammen, den Umfang eines Quinio hatte, vgl. die Hinweise unten, vor allem auf S. 26.

${ }^{35}$ Vgl. das Zitat oben im Abkürzungsverzeichnis in der einleitenden Asteriscus-Anmerkung.

${ }^{36}$ P. D. Eyre, St. Andreas of Crete. A Catalogue of Mss. of his Homilies. Thesis submitted for the degree of M. A. University of Birmingham, Theology Department. Birmingham 1966 (für die freundliche Hilfe bei der Vermittlung dieser Arbeit danken die Autoren Frau Prof. Mary B. Cunningham Corran [University of Birmingham]); vgl. dazu auch J. N. BIRDSAlL, Homilies ascribed to Andreas Cretensis in MS. Halensis A 119, in: J. Dummer(J. Irmscher-F. PAschKe-K. Treu) (Hrsg.) Texte und Textkritik. Eine Aufsatzsammlung (Texte und Untersuchungen zur Geschichte der altchristlichen Literatur 133). Berlin 1987, 49, Anm. 3. (bzw. das Postscript auf S. 51); vgl. auch Detorake (Vollzitat in Anm. 38) 161, Anm. 40.

${ }^{37}$ R. E. Sinkewicz, Manuscript Listings for the Authors of the Patristic and Byzantine Periods (Greek Index Project Series 4). Toronto 1992. - Subsidiär wurden auch die Handschriftenlisten bei De Strycker, Protevangelium 597-607, und bei Kotter, Die Schriften des Johannes von Damaskos V 152-155 (vgl. auch schon a. O. 3-55) herangezogen.

${ }^{38}$ Zusätzlich wurde unter den genannten Gesichtspunkten auch die bei GeERARD, Clavis III 541 („Vorspann“ zum Andreas-Lemma), angeführte Literatur ausgewertet. - Vgl. jetzt

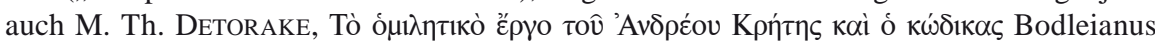

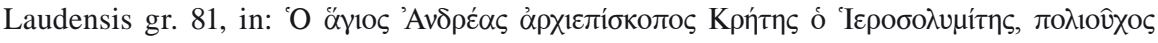

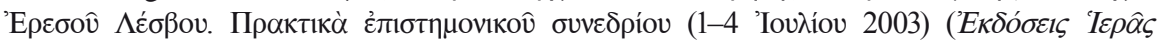

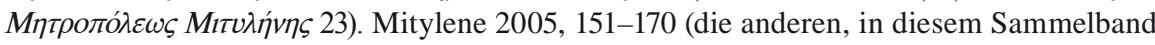
enthaltenen Beiträge bieten keine zusätzlichen, über die von uns eingesehene Literatur hinausgehenden Informationen zur handschriftlichen Überlieferung des Andreas von Kreta).

${ }^{39}$ Die im folgenden angeführten ältesten Zeugen (die allesamt an Hand von Mikrofilmen und Photographien eingesehen wurden) fanden auch bei der provisorischen Textkonstitution der durch die Palimpseste im Cod. Vind. theol. gr. 160 repräsentierten Andreas-Passagen (unten, S. 40-44) Berücksichtigung (aus diesem Grunde werden sie über dem Strich gleich 
Vat. gr. 2079 (V): Jahrespanegyrikon ${ }^{40}$; 9. Jahrhundert (Mitte), Pergament, 208 × 146 mm, frühe (,,studitische“) Minuskel (wohl nicht von der Hand des Nikolaos Studites) (einspaltig). - (f. 109 $\left.-120^{\mathrm{r}}\right)^{41}$ Andreas von Kreta, Homilia I in nativitatem B. M. V. ([f. 120 $\left.-121^{\mathrm{v}}\right]$ Ioannes Chrysostomos, In nativitatem B. $\left.M . V^{42}\right)^{43}$.

Esc. $\Phi-I I I-20$ (gr. 239) (E): Jahrespanegyrikon ${ }^{44}$; 9. Jahrhundert (zweite Hälfte), Pergament, $202 \times 142 \mathrm{~mm}$, rechtsgeneigte ogivale Majuskel (einspaltig). - (f. $\left.1^{\mathrm{r}}-9^{\mathrm{r}}\right)^{45}$ Andreas von Kreta, Homilia I in nativitatem B. M. V. ([f. 9 $\left.{ }^{\mathrm{r}}-20^{\mathrm{r}}\right]$ Theodoros Studites, In nativitatem B. M. $\left.V^{46}\right)^{47}$.

mit ihren im folgenden im Apparat verwendeten Siglen vorgestellt). - In einer weiteren Übersicht (vgl. unten, S. 16-23) sollen in kürzerer Form auch jene jüngeren Codices vorgestellt werden, welche die soeben genannte Überlieferungsgemeinschaft dokumentieren; ferner erschien es auch ratsam, auf jene Handschriften einzugehen, die als Vorlage für die jeweiligen Editionen (de Billy, Combefis, Patusas, Gallandi, Migne) identifiziert werden konnten (vgl. unten, S. 36-38). - Im Falle der Homilia III in nativitatem B. M. V. wurde auf die Tatsache Rücksicht genommen, daß diese Rede, wie bereits angedeutet, bisweilen auch unter dem Namen des Ioannes von Damaskos tradiert wird (vgl. oben, Anm. 19). - Eine abschließende Vorbemerkung: In jenen Fällen, in denen die chronologische Einreihung eines Codex von der in der Literatur vertretenen Datierung abweicht, handelt es sich um eine stillschweigend von uns auf der Grundlage des von uns ausgewerteten Photomaterials vorgenommene Verbesserung.

${ }^{40}$ EHRHARD, Überlieferung I 227-230 (hier und im folgenden wird jeweils die Haupterwähnung des betreffenden Codex im Handbuch von Ehrhard angegeben; sonstige Nennungen lassen sich bequem mit Hilfe von L. PerRIA, I manoscritti citati da Albert Ehrhard [Testi e Studi bizantino-neoellenici IV]. Rom 1979, aufspüren).

${ }^{41}$ Davor: Theodoros Studites, In dormitionem B. M. V. (15. August).

${ }^{42}$ Unediert; vgl. Codices Chrysostomici graeci VI. Codicum civitatis Vaticanae partem priorem descripsit S. J. Voicu (Documents, Études et Répertoires publiés par l'Institut de Recherche et d'Histoire des Textes 11, 6). Paris 1999, 245 (Nr. 19); vgl. auch GEerARD, Clavis II 647 (Nr. 5057; ohne Bewertung der Echtheitsfrage).

${ }^{43}$ Aus der reichen Literatur zu diesem Codex vgl. etwa H. FolLieri, Codices graeci Bibliothecae Vaticanae selecti, temporum locorumque ordine digesti, commentariis et transcriptionibus instructi (Exempla Scripturarum IV). Città del Vaticano 1969, 23 -24 (Nr. 13); Abb. auf Taf. 13; zur Diskussion der Identifizierung des Kopisten des Cod. Vat. gr. 2079 vgl. auch die bei G. De Gregorio, Materiali vecchi e nuovi per uno studio della minuscola greca fra VII e IX secolo, in: I manoscritti greci tra riflessione e dibattito (wie in Anm. 15) 136, Anm. 266, angegebene Literatur. - Eine vollständige, modernen Ansprüchen genügende Katalogisierung des Vaticanus fehlt; die folgenden, für die weitere Beweisführung wichtigen Angaben verdanken wir unserem Freund Giuseppe De Gregorio (der uns auch zu den anderen von uns herangezogenen Codices der Biblioteca Apostolica Vaticana wertvolle, über die gedruckten Kataloge hinausgehende Informationen zur Verfügung gestellt hat): Die uns interessierenden Texte finden sich in zwei (vollständigen, ungestörten) Quaternionen, f. 109-116 und f. 117-124 (Kustoden sind heute nicht mehr sichtbar [es lassen sich aber sowohl auf f. 109r als auch auf f. 117 ${ }^{\mathrm{r}}$ im oberen Freirand - links und rechts - Spuren der für die frühe studitische Handschriftenproduktion charakteristischen Kreuze feststellen], doch kann aus der Tatsache, daß auf f. $41^{\mathrm{r}}$ die Kustode $\zeta^{\prime}$ sichtbar ist, gefolgert werden, daß die beiden zur Diskussion stehenden Quaternionen die 15. und die 16. Lage des Codex bilden). - Für die im folgenden (vgl. S. 25-27) wichtige Bewertung des (auf „Migne-Zeilen“ umgerechneten) Umfangs der unedierten chrysostomischen Homilie ist die Beobachtung ausschlaggebend, daß ihr Text auf f. $120^{\mathrm{r}}$ in der 2. Zeile von unten (Überschrift: 5.-3. Zeile von unten) beginnt und auf f. $121^{\mathrm{v}}$ ganz unten (danach nur noch eine abschließende Zierleiste) endet; sie nimmt also im Vaticanus drei volle Seiten und zwei (bzw. fünf) Zeilen ein. Im Vergleich dazu: Die Homilia I in nativitatem B. M. V. des Andreas von Kreta beansprucht im Cod. Vat. gr. 2079 mehr als 21 volle Seiten.

${ }^{44}$ EHRHARD, Überlieferung II 4-6.

${ }^{45}$ Vor $\mathrm{f} .1$ kein Textverlust.

${ }^{46}$ GeERARD, Clavis III 532 (Nr. 8119). 
Par. gr. 766 (P): Jahrespanegyrikon ${ }^{48}$; 9. Jahrhundert (zweite Hälfte/Ende), Pergament, $295 \times 195$ mm, Weiterentwicklung der frühen Minuskel (einspaltig). - (f. 136r-144v ${ }^{\text {r }}{ }^{49}$ Andreas von Kreta, Homilia I in nativitatem B. M. V.; (f. 145 ${ }^{\text {r }}$ 155') Andreas von Kreta, Homilia III in nativitatem B. M. V. ([f. 156r-159'] [Ps.-]Ioannes Chrysostomos, De exaltatione crucis [14. September] $)^{50}$.

Mosqu. Syn. gr. 284 (Vlad. 215) (M): Jahrespanegyrikon51; 9. Jahrhundert (Ende), Pergament, $267 \times 178 \mathrm{~mm}$, Vorstufe des Kirchenlehrerstils («minuscule bouletée») (einspaltig). - (f. $1^{\mathrm{r}}-4^{\mathrm{v}}$ ) Andreas von Kreta, Homilia I in nati-

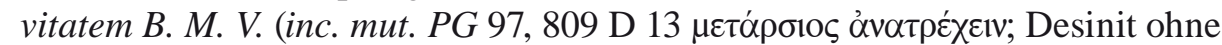
Textverlust) ([f. $\left.9^{\mathrm{r}}-10^{\mathrm{v}} .5^{\mathrm{r}}-8^{\mathrm{r}}\right]$ Andreas von Kreta, Homilia IV in nativitatem

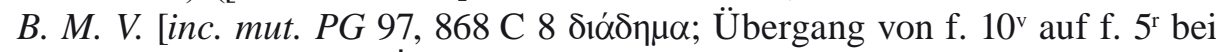

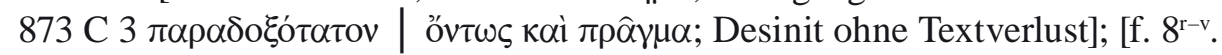
$11^{\mathrm{r}}-17^{\mathrm{v}}$ ] [Ps.-]Ioannes von Damaskos, Oratio in nativitatem sanctae Dei genitricis Mariae $\left.{ }^{52}\right)^{53}$.

${ }^{47}$ G. DE ANDRÉs, Catálogo de los códices griegos de la Real Biblioteca de El Escorial II. Madrid 1965, 77-80; Abb. (f. 45 und f. 33) bei Ch. GrauX-A. MARTin, Fac-similés de manuscrits grecs d’Espagne. Paris 1891, Taf. 3 und 4 (vgl. dazu die Beschreibung: a. O. 7-11); für die Vermittlung von Photomaterial zum Escurialensis danken wir unserem Kollegen Juan Signes. - Ein weiterer (zweispaltiger) Majuskelzeuge (des 9. Jahrhunderts) für die Homilia I in nativitatem B. M. V. des Andreas von Kreta liegt in der unteren, gründlich palimpsestierten Schrift des Cod. Vat. Pal. gr. 205 vor (vgl. EHRHARD, Überlieferung I 95-97): Die wenigen Passagen, die auf f. $160^{\mathrm{r}}+163^{\mathrm{v}}$ bzw. auf f. $160^{\mathrm{v}}+163^{\mathrm{r}}$ und auf f. $161^{\mathrm{r}}+162^{\mathrm{v}}$ bzw. auf f. $161^{\mathrm{v}}+162^{\mathrm{r}}$ zu lesen sind (vgl. etwa den Beginn von Homilia I in nativitatem B. M. V. auf f. 160r [äußere Kolumne einer Recto-Seite der palimpsestierten Handschrift; letzte lesbare Reste in der

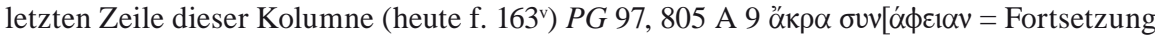
auf f. 160v]; letzte überhaupt erkennbare Buchstabenfragmente auf f. 162 ${ }^{\mathrm{r}}$ : $P G$ 97, 809 D

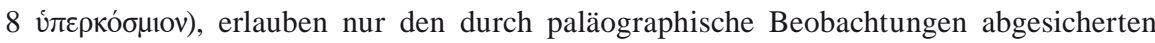
Schluß, daß diese Fragmente nicht jenem alten Codex entstammen, dem die im Cod. Vind. theol. gr. 160 wiederverwendeten Blätter entnommen wurden (die wohl der Mitte oder der zweiten Hälfte des 9. Jahrhunderts entstammende rechtsgeneigte ogivale Majuskel der unteren Schrift der genannten Blätter des Vat. Pal. gr. 205 ist weitaus weniger sorgfältig geschrieben als die stärker nach rechts geneigte untere Schrift der Wiener Fragmente); wichtig sind auch die Hinweise bei EhrHARD, a. O. 96, daß die Palimpsestblätter des Cod. Vat. Pal. gr. 205 einem Homiliar entstammen, das nicht mit dem September begann (vor Texten, die sich auf den 14. September beziehen, dürfte diese Sammlung nicht weniger als 100 Lesungen geboten haben), und daß sich in der unteren Schrift des Vaticanus auch das Protevangelium Iacobi (vgl. oben, Anm. 29) findet (vgl. das von Ehrhard richtig erkannte Incipit auf f. 51 $)$. Ob der „Muttercodex“ dieser Palimpseste einst auch die Homilia III in nativitatem B. M. V. des Andreas von Kreta enthalten hat, läßt sich nicht feststellen.

${ }^{48}$ EHRHARD, Überlieferung II 72-76.

49 Davor (f. $111^{v}-135^{v}$ ): Andreas von Kreta, Homilia in dormitionem B. M. V. (15. August).

${ }^{50} \mathrm{H}$. OMONT, Inventaire sommaire des manuscrits grecs de la Bibliothèque Nationale I. Paris 1886, 132-133; F. HALKIn, Manuscrits grecs de Paris. Inventaire hagiographique (Subsidia Hagiographica 44). Bruxelles 1968, 63-64. - Für die Vermittlung von Photographien des Par. gr. 766 (und anderer Parisini) danken wir unserem Freund Michael Featherstone und Herrn Dr. Chr. Foerstel.

${ }^{51}$ EHRHARD, Überlieferung II 6-9.

52 Geerard, Clavis II 519 (Nr. 8060); vgl. auch oben, Anm. 29.

${ }^{53}$ Vgl. Chr. Fr. DE MATtheI, Accurata codicum graecorum mss. Bibliothecarum Mosquensium Sanctissimae Synodi notitia et recensio I. Leipzig 1805, 169-171; Archimandrit VLADIMIR, Sistematičeskoe opisanie rukopisej Moskovskoj Sinodal'noj (Patriaršej) Biblioteki I. Rukopisi grečeskija. Moskva 1894, 262-267; KotTER, Die Schriften des Johannes von Damaskos V 31 (Nr. 245) und 154; s. auch M. L. AgATI, La minuscola «bouletée» (Littera Antiqua 9, 1-2). Città del Vaticano 1992, 269-270 (mit Abb. auf Taf. 184). - Für die Überlassung entsprechender Abzüge von einem Mikrofilm danken wir Herrn Dr. Robert Volk (Arbeitsstelle Scheyern der Patristischen Kommission der Bayerischen Akademie der 
Vat. gr. 455 (G): Halbjahrespanegyrikon ${ }^{54}$; 10. Jahrhundert (Anfang/erste Hälfte), Pergament, $362 \times 254$ mm, Minuskel (mit einer gewissen Nähe zu Codices, die für Arethas von Kaisareia geschrieben wurden ${ }^{55}$ ) (zweispaltig). - (f. 208 ${ }^{\text {r }}$ $\left.213^{r}\right)^{56}$ Andreas von Kreta, Homilia I in nativitatem B. M. V. ([f. 213 ${ }^{\mathrm{r}}-220^{\mathrm{r}}$ ] Andreas von Kreta, Homilia IV in nativitatem B. M. $V_{.^{57}}^{58}$.

Par. gr. 763 (D): homiletische Sammelhandschrift ${ }^{59}$; 10. Jahrhundert (erste Hälfte), Pergament, $310 \times 210 \mathrm{~mm}$, unspezifische Weiterentwicklung der frühen Minuskel ${ }^{60}$ (zweispaltig). - (f. 23v-30r ${ }^{61}$ Andreas von Kreta, Homilia I in nativitatem B. M. V. ([f. 30-39v] Basileios von Kaisareia, Quod Deus non est auctor malorum $\left.{ }^{62}\right)^{63}$.

Par. gr. 1171 (K): Halbjahrespanegyrikon ${ }^{64}$; 10. Jahrhundert (erste Hälfte), Pergament, $325 \times 220 \mathrm{~mm}$, unspezifische Weiterentwicklung der frühen Minuskel auf relativ niedrigem Niveau ${ }^{65}$ (einspaltig). - (f. 42 $\left.-46^{v}\right)^{66}$ Andreas von Kreta,

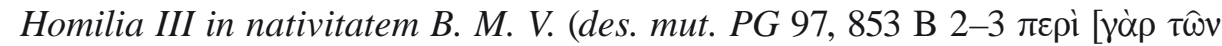
$\left.{ }^{\prime \prime} \lambda \lambda \omega v^{67}\right)^{68}$. cherche et d'Histoire des Textes (Paris) aufbewahrte Mikrofilmmaterial zum Mosquensis (und zu dem im folgenden behandelten Cod. Sinait. gr. 749) vermittelte.

${ }^{54}$ EHrhard, Überlieferung II 113-119.

55 Vgl. etwa den Cod. Vat. Urb. gr. 35 (vgl. Taf. 13 bei L. Th. LEForT-J. Cochez, Palaeographisch album van gedagteekende grieksche minuskelhandschriften uit de $\mathrm{IX}^{\mathrm{e}}$ en $\mathrm{X}^{\mathrm{e}}$ eeuw. Leuven 1932); die in einem Teil der Literatur zum Cod. Vat. gr. 455 postulierte süditalienische Provenienz (z. B. De Strycker, Protevangelium 600, oder bei Voicu, Codices Chrysostomici graeci [wie in Anm. 42] VI 36) geht auf eine etwas weitherzige Auslegung der Angaben im Katalog von Devreesse (Vollzitat unten in Anm. 58) zurück; skeptisch bereits EHRHARD, Überlieferung II 114, Anm. 2 (von S. 113).

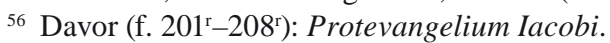

57 Danach finden sich zum 8. September noch folgende Texte: (f. 220 $\left.{ }^{r}-226^{v}\right)$ (Ps.-)Ioannes von Damaskos, Oratio in nativitatem sanctae Dei genitricis Mariae, (f. 226 $-232^{v}$ ) Theodoros Studites, In nativitatem B. M. V.

${ }^{58}$ R. DevreEsse, Codices Vaticani graeci II. Codices 330-603. Città del Vaticano 1937, 210-215; KotTeR, Die Schriften des Johannes von Damaskos V 44 (Nr. 349) und 158.

${ }^{59}$ Nicht bei EHRHARD, Überlieferung, besprochen.

${ }^{60}$ Diese Aussage gilt für den ersten Teil des Codex (f. $\left.1^{\mathrm{r}}-198^{v}\right)$; der zweite Teil ist in einer «minuscule bouletée» („Kirchenlehrerstil“) geschrieben: vgl. AGATI, La minuscola «bouletée» (wie in Anm. 53) 69 (mit Abb. auf Taf. 34) (die erste Hand charakterisiert Agati, a. O. 69, als «agile minuscola rotonda, da nuclei leggermente schiaccati e geometrizzanti ..., comunque contemporanea all'altra»); für eine süditalienische Provenienz plädiert (wohl nicht zu Recht) J. IRIGOIN, L'Italie méridionale et la tradition des textes antiques. Jahrbuch der Österreichischen Byzantinistik 18 (1969) 46.

${ }^{61}$ Davor (f. 19r-23v): (Ps.-)Ioannes Chrysostomos, Homilia in decollationem Iohannis Baptistae (29. August).

${ }^{62}$ GeERARd, Clavis II 147 (Nr. 2853).

${ }^{63}$ Omont, Inventaire sommaire (wie in Anm. 50) I 131; Halkin, Manuscrits grecs de Paris (wie in Anm. 50) 62.

${ }^{64}$ EHRHARD, Überlieferung I 281-285.

${ }^{65}$ Diese Aussage gilt wiederum nur für den ersten Teil des Codex (f. $1^{\mathrm{r}}-274^{v}$ ); der zweite Teil ist in einer «minuscule bouletée» („Kirchenlehrerstil“) geschrieben: vgl. AGATI, La minuscola «bouletée» (wie in Anm. 53) 58 (mit Abb. auf Taf. 28) (die erste Hand charakterisiert AgATI, a. O. 58, als «appesa al rigo e rotonda, comunque coeva»).

${ }^{66}$ Davor (f. 35r-42v): (Ps.-)Ioannes von Damaskos, Oratio in nativitatem sanctae Dei genitricis Mariae.

${ }^{67}$ Der Textausfall zwischen f. $46^{v}$ und f. $47^{\mathrm{r}}$ läßt sich umfangmäßig nicht genauer bestimmen: vgl. die Hinweise bei EHRHARD, Überlieferung I 282, Anm. 1 (der nächste erhaltene Text gehört zum 8. November); theoretisch nicht auszuschließen ist die Vermutung, daß in der 
Sinait. gr. 749 (526) (A): Halbjahrespanegyrikon ${ }^{69}$; 10. Jahrhundert (Mitte/ zweite Hälfte), Pergament, $290 \times 225$ mm, primitiver Vorläufer der Perlschrift (zweispaltig). - (f. $\left.7^{\mathrm{r}}-11^{\mathrm{v}}\right)^{70}$ Andreas von Kreta, Homilia I in nativitatem B. M. V.

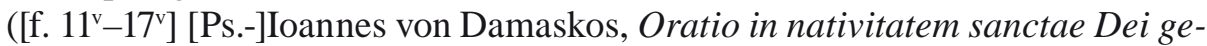
nitricis Mariae $)^{71}$.

Par. gr. 1454 (C): Halbjahrespanegryikon ${ }^{72}$; 10. Jahrhundert (zweite Hälfte), Pergament, $360 \times 250$ mm, unspezifische Minuskel (in der Art einer «corsiveggiante inclinata») (zweispaltig). - (f. 31 $\left.{ }^{\mathrm{v}}-36^{\mathrm{r}}\right)^{73}$ Andreas von Kreta, Homilia I in nativitatem B. M. V. ([f. 36 $\left.{ }^{\mathrm{r}}-42^{\mathrm{r}}\right]$ Vita Theodorae Alexandrinae [11. September] $)^{74}$.

Diese (wie gesagt: nicht auf absolute Vollständigkeit abzielende) Übersicht der ältesten Textzeugen zeigt, daß sich in den codices vetusti ausschließlich im Cod. Par. gr. 766 eine Überlieferungsgemeinschaft nachweisen läßt, in der die Homilia III in nativitatem B. M. V. des Andreas von Kreta unmittelbar auf dessen Homilia I folgt, in der also eine Tradition vorliegt, welche die oben vermutete Rekonstruktion jener Lage, der die Palimpsestfragmente im Cod. Vind. theol. gr. 160 entstammen, als Ternio untermauern könnte. Darüber hinaus läßt sich die Überlieferungsgemeinschaft der ersten und der dritten Homilie des Andreas von Kreta auf Mariae Geburt in folgenden jüngeren Codices belegen (wobei zwischen Handschriften differenziert wird, in denen die beiden Homilien unmittelbar aufeinanderfolgen, und solchen, in denen zwischen der Homilia I und der Homilia III ein anderer Text bzw. andere Texte [mit einem Bezug auf den 8. September] eingeschoben ist [sind $])^{75}$.

In direktem Anschluß an die Homilia I folgt die Homilia III im Cod. Ambros. C 123 sup. aus dem 11./12. Jahrhundert ${ }^{76}$ (f. $1^{\mathrm{r}}$ [inc. mut.] $-5^{\mathrm{r}}$ und f. $5^{\mathrm{r}}-$ ?) ${ }^{77}$, im Cod. Messin. Univ. S. Salv. 4 aus dem 12.(/13.) Jahrhundert (f. $1^{\mathrm{r}}-7^{\mathrm{v}}$ und

Textlücke des Parisinus die Homilia I in nativitatem B. M. V. des Andreas von Kreta Platz gefunden hatte, da Codices ab dem 12. Jahrhundert auch eine Reihenfolge Homilia III und dann Homilia I in nativitatem B. M. V. des Andreas von Kreta belegen; vgl. gleich im folgenden.

${ }^{68}$ Omont, Inventaire sommaire (wie in Anm. 50) I 234-235; Halkin, Manuscrits grecs de Paris (wie in Anm. 50) 119-120; KотTER, Die Schriften des Johannes von Damaskos V 37 (Nr. 290) und 166.

${ }^{69}$ EHRHARD, Überlieferung I 239-246.

${ }^{70}$ Davor (f. $1^{\mathrm{r}-7 \mathrm{r}}$ ): Antonios (Hagiograph), Vita Symeonis Stylitae senioris (inc. mut.; Desinit wie BHG 1685) (1. September).

${ }^{71}$ A. VASIL’Ev, Zamětki o někotorych grečeskich rukopisjach žitij svjatych na Sinaě. Vizantijskij Vremennik 14 (1907) 288-292; KotTER, Die Schriften des Johannes von Damaskos V 50 (Nr. 389) und 155.

72 EHRHARD, Überlieferung I 234-239.

${ }^{73}$ Davor (f. 24v-31'): Protevangelium Iacobi.

${ }^{74}$ Omont, Inventaire sommaire (wie in Anm. 50) II 48; HaLkin, Manuscrits grecs de Paris (wie in Anm. 50) 164.

${ }^{75}$ Wiederum gilt der Hinweis, daß in den beiden folgenden Übersichten keine Vollständigkeit angestrebt wurde.

${ }^{76}$ In diesem Fall (und in allen folgenden Fällen) folgen wir den Datierungsansätzen in der vorhandenen Literatur, da wir in die betreffenden Handschriften keine Einsicht genommen haben; eine analoge Kautel gilt auch für die Folienangaben (der in diesem Punkte, etwa im Hinblick auf Recto- und Verso-Seiten, nicht immer vollständigen oder genauen älteren Literatur), die wir nicht eigens überprüft haben.

77 Ehrhard, Überlieferung I 306-308 (mit Hinweis auf eine süditalienische Provenienz); Ae. MARTINI-D. BASSI, Catalogus codicum graecorum Bibliothecae Ambrosianae I. Milano 1906, 226-228 (ohne Angabe des Blattes, auf dem Homilia III endet). 
f. $\left.7^{\mathrm{v}}-15^{\mathrm{r}}\right)^{78}$, im Cod. Oxon. Bodl. Auct. E. 2. 6 (Misc. 34) aus dem 12. Jahrhundert (f. $1^{\mathrm{r}}-7^{\mathrm{r}}$ und f. $\left.7^{\mathrm{v}}-15^{\mathrm{r}}\right)^{79}$, im Cod. Ambros. C 124 sup. aus dem 13. Jahrhundert (f. $8^{\text {r }}-13^{r}$ und f. $\left.13^{r}-19^{r}\right)^{80}$, im Cod. Athous Meg. Laur. $\Delta 78$ aus dem 13./14. Jahrhundert (f. $20^{\text {r }}-25^{r}$ und f. $\left.25^{r}-32^{r}\right)^{81}$, (wahrscheinlich) im Cod. Patm. 380 vom Jahre 1544 (von der Hand eines Nikolaos Anagnostes) ${ }^{82}$, im Cod. Athen. Ethn. Bibl. 217 aus dem 17. Jahrhundert ${ }^{83}$ und im Cod. Athous Meg. Laur. E 160 aus dem Jahre 1705 (f. 21 $21^{\text {r }}$ ? $)^{84}$.

Für eine „invertierte“ ${ }^{\text {85 }}$ Ordnung (Homilia III in nativitatem B. M. V. des Andreas von Kreta unmittelbar vor seiner Homilia I) läßt sich der Cod. Lesb.

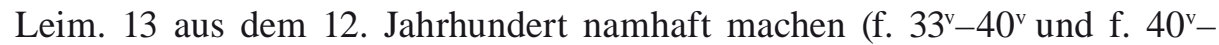

${ }^{78}$ Ehrhard, Überlieferung II 186-188 (wieder mit Hinweis auf eine süditalienische Provenienz); A. Mancinı, Codices graeci monasterii S. Salvatoris. Atti della Reale Accademia Peloritana 22, Fasz. 2 (1907) 11-14. - Nach der Homilia III folgt zum 8. September noch (f. $15^{\mathrm{r}}-25^{\mathrm{r}}$ ) die Homilia II in nativitatem B. M. V. des Andreas von Kreta (GEERARD, Clavis III 542 [Nr. 8171]).

${ }^{79}$ EHRHARD, Überlieferung II 121-124; H. O. CoXE, Catalogi codicum manuscriptorum Bibliothecae Bodleianae I. Oxford 1853, 637-640; KotTER, Die Schriften des Johannes von Damaskos V 34 (Nr. 270) und 164; vgl. auch C. VAn DEN Vorst-H. Delehaye, Catalogus codicum hagiographicorum graecorum Germaniae, Belgii, Angliae (Subsidia Hagiographica 13). Bruxelles 1913, 362-363. - Nach der Homilia III folgen zu diesem Marienfesttag noch die (f. $15^{r}-25^{r}$ ) Homilia II und (f. 25r-33r) Homilia IV in nativitatem B. M. V. des Andreas von Kreta (GEeRARD, Clavis III 542-543 [Nr. 8173]) und (f. 33r-41') (Ps.-)Ioannes von Damaskos, Oratio in nativitatem sanctae Dei genitricis Mariae.

${ }^{80}$ Ehrhard, Überlieferung III 212; Martini-Bassi, Catalogus (wie in Anm. 77) 228-230; KotTER, Die Schriften des Johannes von Damaskos V 37 (Nr. 290) und 166. - Vor Homilia I findet sich im Ambrosianus (f. $1^{\mathrm{r}}-8^{\mathrm{r}}$ ) die Homilia IV in nativitatem B. M. V. des Andreas von Kreta, nach der Homilia III (f. 19-26r) die Homilia II in nativitatem B. M. V. des Andreas, (f. 26r-26') Ioannes Chrysostomos, In nativitatem B. M. V. und (f. 26 $-32^{\mathrm{r}}$ ) (Ps.-)Ioannes von Damaskos, Oratio in nativitatem sanctae Dei genitricis Mariae.

${ }^{81}$ EHRHARD, Überlieferung III 171-173; SPYRIDON LAURIOTES-S. EUSTRATIADES, Catalogue of the Greek Manuscripts in the Library of the Laura on Mount Athos (Harvard Theological Studies XII). Cambridge 1925, 69-70; KotTER, Die Schriften des Johannes von Damaskos V 12 (Nr. 90) und 165. - Im athonitischen Codex lautet die Textabfolge: (f. $8^{\mathrm{r}}-14^{\mathrm{r}}$ ) Theodoros Studites, In nativitatem B. M. V., (f. 14r-20') (Ps.-)Ioannes von Damaskos, Oratio in nativitatem sanctae Dei genitricis Mariae, (f. $20^{\mathrm{r}}-25^{\mathrm{r}}$ ) Andreas von Kreta, Homilia I in nativitatem B. M. V., (f. 25r-32r) Andreas von Kreta, Homilia III in nativitatem B. M. V., (f. 32 $2^{r}-38^{r}$ ) Andreas von Kreta, Homilia II in nativitatem B. M. V., (f. 38-44r) Andreas von Kreta, Homilia IV in nativitatem B. M. V.

${ }^{82}$ EHRHARD, Überlieferung II 125-129; entsprechende Folienangaben fehlen bei Ehrhard und

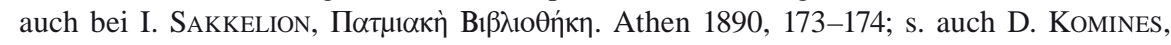

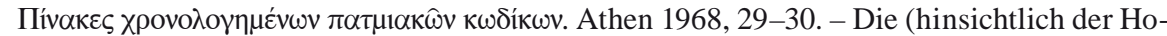
milien des Andreas von Kreta von Ehrhard nach dem Cod. Oxon. Bodl. Auct. E. 2. 6 rekonstruierte) Textabfolge (vgl. Ehrhard, a. O. 125-126) lautet hier: (f. $1^{\mathrm{r}}-10^{\mathrm{r}}$ ) Protevangelium Iacobi, Homilia I, Homilia III, Homilia II, Homilia IV und Proklos von Konstantinopel, Homilia de laudibus B. M. V. (GeERARD, Clavis III 133 [Nr. 5800]).

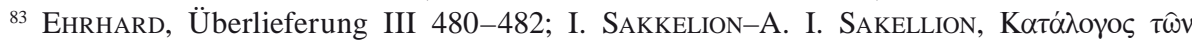

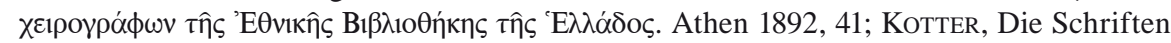
des Johannes von Damaskos V 4 (Nr. 3) und 165. - Reihenfolge der Texte: Homilia I, Homilia III, Homilia IV, (p. 137-150) (Ps.-)Ioannes von Damaskos, Oratio in nativitatem sanctae Dei genitricis Mariae, Theodoros Studites, In nativitatem B. M. V.

${ }^{84}$ EhrHARD, Überlieferung III 173; SpYridon LAURIOTES-Eustratiades, Catalogue Laura (wie in Anm. 81) 93-94; Kotтer, Die Schriften des Johannes von Damaskos V 13 (Nr. 94). - Die Textabfolge zum 8. September lautet hier: (f. $7^{\mathrm{r}}-14^{\mathrm{r}}$ ) Theodoros Studites, In nativitatem B. M. V., (f. 14 ${ }^{\mathrm{r}}-21^{\mathrm{r}}$ ) (Ps.-)Ioannes von Damaskos, Oratio in nativitatem sanctae Dei genitricis Mariae, (f. 21 ${ }^{\text {r}}-51^{\text {r) }}$ ) Andreas von Kreta, Homilia I, Homilia III, Homilia II, Homilia IV in nativitatem B. M. V.

${ }^{85}$ Vgl. unsere oben in Anm. 20 geäußerten Vorbehalte gegen die künstliche, erst bei Migne vorzufindende Zählung der Marienhomilien des Andreas von Kreta. 
46 ${ }^{\mathrm{v}}{ }^{86}$; dazu treten der Cod. Oxon. Bodl. Seld. 46 (= Arch. Seld. B 53), wohl aus dem ausgehenden 13. oder aus dem frühen 14. Jahrhundert (f. $9^{\mathrm{r}}-16^{\mathrm{v}}$ und f.

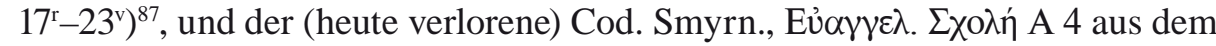
16. Jahrhundert ${ }^{88}$.

Einschübe anderer (scil. auf den 8. September bezüglicher) Texte zwischen der Homilia I und der Homilia III in nativitatem B. M. V. des Andreas von Kreta lassen sich in folgenden Codices feststellen: Cod. Laur. IV 4 aus dem 12.

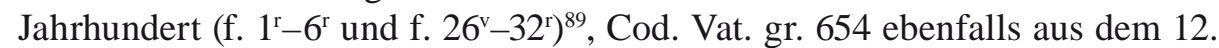
Jahrhundert (f. $1^{\mathrm{r}}-6^{\mathrm{v}}$ und f. $\left.36^{\mathrm{r}}-44^{\mathrm{v}}\right)^{90}$, Cod. Vat. gr. 1882 (in dem uns interes-

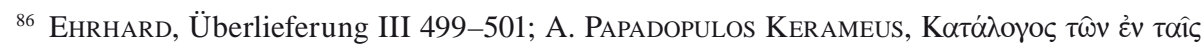

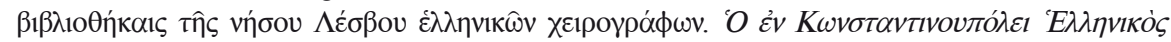

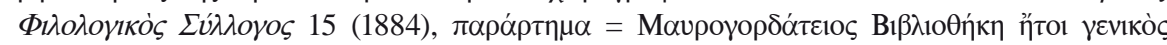

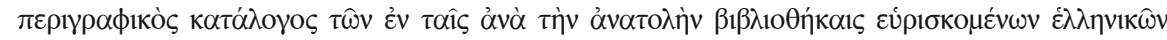

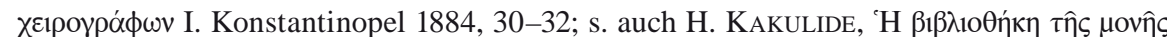

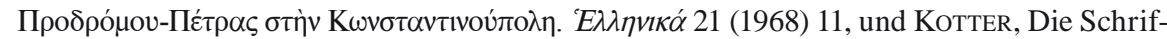
ten des Johannes von Damaskos V 50 (Nr. 198) und 154. - Die Textabfolge lautet hier: (f. $7^{\mathrm{v}}-15^{\mathrm{v}}$ ) (Ps.-)Ioannes von Damaskos, Oratio in nativitatem sanctae Dei genitricis Mariae, (f. $15^{\mathrm{v}}-23^{\mathrm{v}}$ ) Andreas von Kreta, Homilia IV in nativitatem B. M. V. (unter dem Namen des Ioannes von Damaskos), (f. 23-33) Andreas von Kreta, Homilia II in nativitatem B. M. V. (unter dem Namen des Ioannes von Damaskos), (f. 33ㄴ -40v) Andreas von Kreta, Homilia III in nativitatem B. M. V. (unter dem Namen des Ioannes von Damaskos), (f. 40v-46v) Andreas von Kreta, Homilia I in nativitatem B. M. V., (f. 46ㄴ -54v) Protevangelium Iacobi.

87 EhrHard, Überlieferung III 285-287 (mit Hinweis auf den archaisierenden Charakter der Minuskel des Oxoniensis); Coxe, Catalogi (wie in Anm. 79) I 607-610 (datiert „sec. forsan XII exeuntis“); vgl. auch VAn DE VORST-Delehaye, Catalogus (wie in Anm. 79) 346-348

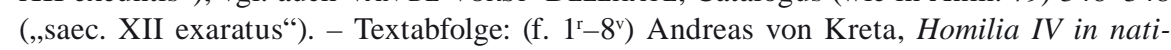
vitatem B. M. V. (unter dem Namen des Ioannes von Damaskos), (f. 9 $9^{\mathrm{r}}-16^{\mathrm{v}}$ ) Andreas von Kreta, Homilia III in nativitatem B. M. V. (unter dem Namen des Ioannes von Damaskos), (f.

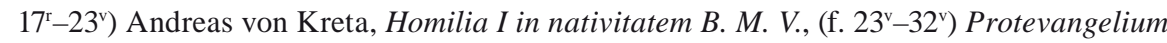
Iacobi, (f. 33-43 ${ }^{\text {r }}$ ) Andreas von Kreta, Homilia II in nativitatem B. M. V. (unter dem Namen des Ioannes von Damaskos).

${ }^{88}$ Ehrhard, Überlieferung III 160-163 (mit Datierung ins 16. Jahrhundert); A. PAPADOPULOS-

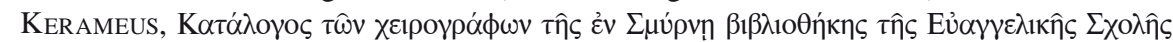

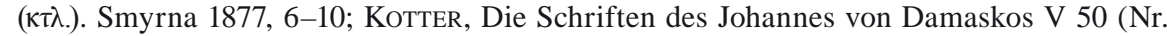
$\langle 393\rangle$ ) und 155. - Textabfolge: Protevangelium Iacobi, (Ps.-)Ioannes von Damaskos, Oratio in nativitatem sanctae Dei genitricis Mariae, Andreas von Kreta, Homilia III in nativitatem B. M. V., Andreas von Kreta, Homilia I in nativitatem B. M. V., Andreas von Kreta, Homilia IV in nativitatem $B . M$. $V$.

89 Ehrhard, Überlieferung II 93-95 (mit Datierung ins 12. Jahrhundert); A. M. BAndinI, Catalogus codicum manuscriptorum Bibliothecae Mediceae Laurentianae. Florenz 1764, 519-523; KotTer, Die Schriften des Johannes von Damaskos V 20 (Nr. 155) und 153. Textabfolge: (f. $1^{\mathrm{r}}-6^{\mathrm{r}}$ ) Andreas von Kreta, Homilia I in nativitatem B. M. V., (f. 6r-12 ${ }^{\mathrm{v}}$ ) (Ps.-) Ioannes von Damaskos, Oratio in nativitatem sanctae Dei genitricis Mariae, (f. 13r-19²) Andreas von Kreta, Homilia IV in nativitatem B. M. V. (unter dem Namen des Ioannes von Damaskos), (f. 19-26v) Andreas von Kreta, Homilia II in nativitatem B. M. V. (unter dem Namen des Ioannes von Damaskos), (f. 26-32 ) Andreas von Kreta, Homilia III in nativitatem B. M. V. (unter dem Namen des Ioannes von Damaskos).

90 Ehrhard, Überlieferung II 91-93; R. Devreesse, Codices Vaticani graeci III. Codices 604-866. Città del Vaticano 1950, 82-85; KотTER, Die Schriften des Johannes von Damaskos V 45 (Nr. 352) und 159. - Textabfolge: (f. $1^{\text {r}}-6^{v}$ ) Andreas von Kreta, Homilia I in nativi-

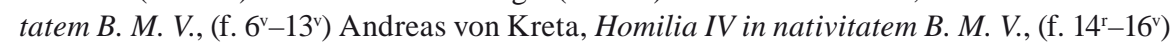
Kosmas Bestitor, Encomium II in s. Zachariam (GEerARD, Clavis III 540 [Nr. 8153]), (f.

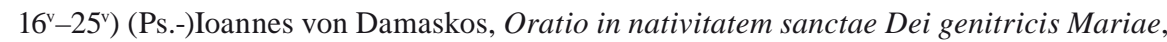
(f. $25^{\mathrm{v}}-36^{\mathrm{r}}$ ) Andreas von Kreta, Homilia II in nativitatem B. M. V. (unter dem Namen des Ioannes von Damaskos), (f. 36r-44v) Andreas von Kreta, Homilia III in nativitatem B. M. V. (unter dem Namen des Ioannes von Damaskos), (f. 44ㄴ-54 $)$ Protevangelium Iacobi. - Die Tatsache, daß wir mit dem Enkomion des Kosmas Bestitor auf Zacharias im unbeweglichen Kirchenjahr beim 5. September stehen (während sich die Texte davor und danach auf Mariä 
sierenden Teil) „saec. XII-XIII“ (f. 181 ${ }^{\mathrm{r}-\mathrm{v}}$. 185 $185^{\mathrm{r}}-186^{\mathrm{v}}$. 182 ${ }^{\mathrm{r}-\mathrm{v}}$ [inc. mut. und des. mut.] und f. $\left.150^{\mathrm{r}}-157^{\mathrm{r}}\right)^{91}$, Cod. Athous Vatop. 454 aus dem 13. Jahrhundert ${ }^{92}$, Cod. Vat. Reg. gr. $15^{93}$ aus der zweiten Hälfte des 13. Jahrhunderts (f. $1^{\mathrm{r}}-7^{\mathrm{v}}$ und f. 25r-32v ${ }^{94}$, Cod. Athous Prot. 57 (Lambros 57) aus dem Jahre 1318/1319 (von der Hand eines Mönches Anastasios) ${ }^{95}$, Cod. Athen. Ethn. Bibl. 2734 (ex

Geburt [8. September] beziehen), führt EHRHARD, a. O. 93, ohne nähere Begründung „auf den Schreiber von V“ (= Cod. Vat. gr. 654) zurück. Zu denken gibt freilich die Bemerkung von Devreesse, a. O. 85, daß auf f. 14r eine Lagenzählung mit der Kustode $\alpha^{\prime}$ einsetzt (vgl. auch schon EHRHARD, a. O. 91, Anm. 1), daß also die Zugehörigkeit der ersten 13 Blätter mit den Homiliae I et IV in nativitatem B. M. V. des Andreas von Kreta zum Rest des Codex nicht über jeden Zweifel erhaben ist. Ob in den Folien 1-13 eine (spätere?) Ergänzung des Hauptblocks vorliegt (womit die hier zu untersuchende Überlieferungsgemeinschaft der Homilia I und der Homilia III des Andreas im Vat. gr. 654 nur eine zufällige werden würde), ließe sich nur nach einer autoptischen Untersuchung der vatikanischen Handschrift feststellen (Angaben zu einem allfälligen Handwechsel finden sich weder bei Ehrhard noch bei Devreesse).

${ }^{91}$ Ehrhard, Überlieferung II 206-208; P. CANART, Codices Vaticani graeci. Codices 1745-1962, Bd. I. Codicum enarrationes. Città del Vaticano 1970, 482-483 (Nr. XI; vgl. vor allem die Rekonstruktion der korrekten Blattabfolge auf S. 482). - Textabfolge: (f. 175'-

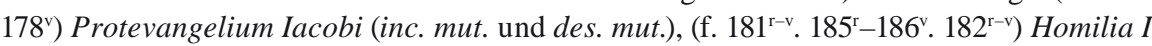
in nativitatem B. M. V. des Andreas von Kreta (inc. mut. und des. mut.), (f. 125r-130v) (Ps.-) Epiphanios von Kypros, Homilia V in laudes B. M. V. (inc. mut.) (GeERARD, Clavis II 335 [Nr. 3771]), (f. 130 ${ }^{\vee}-133^{v}$ ) Kyrillos von Alexandreia, Encomium in B. M. V. (mit Textverlust im Inneren des Textes; des. mut.) (GEeRARD, Clavis III 23-24 [Nr. 5255]), (f. 134-136') Proklos von Konstantinopel, Homilia de laudibus B. M. V. (inc. mut.), (f. 136 ${ }^{\mathrm{r}}-142^{\mathrm{r}}$ ) Andreas von Kreta, Homilia IV in nativitatem B. M. V. (mit Textverlust im Inneren des Textes), (f. 142 ${ }^{\mathrm{r}}-150^{\mathrm{r}}$ ) Andreas von Kreta, Homilia II in nativitatem B. M. V. (mit Textverlust im Inneren des Textes), (f. 150 ${ }^{\mathrm{r}}-157^{\mathrm{r}}$ ) Andreas von Kreta, Homilia III in nativitatem B. M. V., (f. 157-164v) Theodoros Studites, In nativitatem B. M. V. (unter dem Namen des Ioannes von Damaskos).

${ }^{92}$ Ehrhard, Überlieferung III 470-471 (unter der Signatur „Cod. Athon. Batopedi 376“); S. Eustratiades-Arcadios 〈Vatopedinos〉, Catalogue of the Greek Manuscripts in the Library of the Monastery of Vatopedi on Mt. Athos (Harvard Theological Studies XI). Cambridge-Paris-London 1924, 91; KotTER, Die Schriften des Johannes von Damaskos V 7 (Nr. 39C) und 158. - Textabfolge (Folienangaben finden sich nur dort, wo sie mit Sicherheit der Sekundärliteratur entnommen werden konnten): Andreas von Kreta, Homilia I in nativitatem B. M. V., Andreas von Kreta, Homilia IV in nativitatem B. M. V., (f. $18^{r}-26^{v}$ ) (Ps.-)Ioannes von Damaskos, Oratio in nativitatem sanctae Dei genitricis Mariae, Andreas von Kreta, Homilia II in nativitatem B. M. V. (unter dem Namen des Ioannes von Damaskos), Andreas von Kreta, Homilia III in nativitatem B. M. V. (unter dem Namen des Ioannes

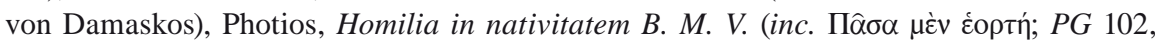

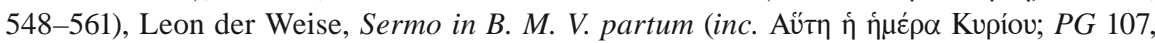
1-12), (f. 57v-70') Protevangelium Iacobi, Proklos von Konstantinopel, Homilia de laudibus B. M. V.

${ }^{93}$ Höchstwahrscheinlich Druckvorlage für die Ausgabe von Combefis (1648): vgl. unten, S. 36.

${ }^{94}$ Ehrhard, Überlieferung III 470-471; H. STEVEnSON, Codices manuscripti graeci reginae Suecorum et Pii pp. II. Rom 1888, 10-12; KotTER, Die Schriften des Johannes von Damaskos V 49 (Nr. 383) und 166. - Textabfolge: (f. $1^{\text {r }}-7^{\mathrm{v}}$ ) Andreas von Kreta, Homilia I in nativitatem B. M. V., (f. $7^{\mathrm{v}}-10^{\mathrm{v}}$ ) Andreas von Kreta, Homilia IV in nativitatem B. M. V. (mit Textverlust im Inneren der Homilie und mit leicht abweichendem Schluß), (f. 11 $11^{\mathrm{r}}-14^{\mathrm{v}}$ ) (Ps.-) Ioannes von Damaskos, Oratio in nativitatem sanctae Dei genitricis Mariae (inc. mut.; mit Textverlusten im Inneren der Homilie; des. mut.), (f. 15r-25r) Andreas von Kreta, Homilia II in nativitatem B. M. V. (unter dem Namen des Ioannes von Damaskos) (inc. mut.), (f. 25r$32^{v}$ ) Andreas von Kreta, Homilia III in nativitatem B. M. V. (unter dem Namen des Ioannes

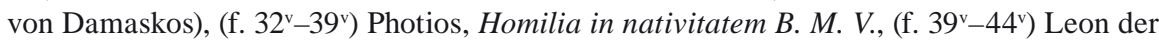
Weise, Sermo in B. M. V. partum, (f. 44 $-53^{v}$ ) Protevangelium Iacobi, (f. 53ㄴ $-58^{r}$ ) Proklos von Konstantinopel, Homilia de laudibus B. M. V.

${ }^{95}$ Ehrhard, Überlieferung II 203-205 (mit Datierung „s. 13-14“); Sp. LAmBros, Catalogue of the Greek Manuscripts on Mount Athos I. Cambridge 1895, 8-9 (mit Datierung ins 14. 
Kastoria 14) aus dem 14. Jahrhundert ${ }^{96}$, Cod. Chalc. Panag. Kamariotisses 1 aus dem Jahre 1360 (von der Hand eines Mönches Iakobos) (f. $1^{\mathrm{r}}-5^{\mathrm{r}}$ und f. $20^{\mathrm{r}}-$ $\left.24^{v}\right)^{97}$, Cod. Par. gr. $819^{98}$ aus dem 16. Jahrhundert (f. $73^{\mathrm{r}}-77^{\mathrm{v}}$ und f. $\left.93^{\mathrm{v}}-98^{\mathrm{v}}\right)^{99}$, Cod. Par. Coisl. 274 aus dem Jahre 1608 (von der Hand des Hieromonachos

Jahrhundert und ohne Angabe von Folien); die chronologische Einreihung und die Zuweisung an den Kopisten Anastasios (auf dem in Verlust geratenen Schlußblatt des Codex) nach den (Ehrhard bekannten! Vgl. Ehrhard, Überlieferung II 205, Anm. 1, und 206, Anm. 1) Kolophonen in zwei jungen Abschriften des athonitischen Codex, im Cod. Par. Coisl. 274 und im Cod. Berol. gr. 43 (Phill. 1447), in denen der Schreiber der recentissimi, Gabriel Sumakupas (s. gleich im folgenden), diese Details zu seinem Antigraphon (auf der Grundlage des zu seiner Zeit [1608] noch erhaltenen Schlußblattes des Cod. Athous Prot. 57) mitteilt (die Hinweise bei EHRHARD, a. O. 205, etwas widersprüchlich, da er bei der Interpretation der Informationen der jungen Abschriften zu ihrer Vorlage - zu einem Codex $\tau \hat{\uparrow} \varsigma$ П $\alpha v \alpha \gamma i \alpha \varsigma$ auf der Insel Chalke - den heute auf dem Athos im Protaton aufbewahrten Codex mit dem Cod. Chalc. Panag. Kamariotisses 47 konfundieren dürfte); KotTer, Die Schriften des Johannes von Damaskos V 17 (Nr. 130). - Textabfolge: Andreas von Kreta, Homilia I in nativitatem B. M. V., (Ps.-)Ioannes von Damaskos, Oratio in nativitatem sanctae Dei genitricis Mariae, (Ps.)Epiphanios von Kypros, Homilia V in laudes B. M. V., Kyrillos von Alexandreia, Encomium in B. M. V., Proklos von Konstantinopel, Homilia de laudibus B. M. V., Andreas von Kreta, Homilia IV in nativitatem B. M. V., Andreas von Kreta, Homilia II in nativitatem B. M. V., Andreas von Kreta, Homilia III in nativitatem B. M. V., Theodoros Studites, In nativitatem B. M. V. (unter dem Namen des Ioannes von Damaskos).

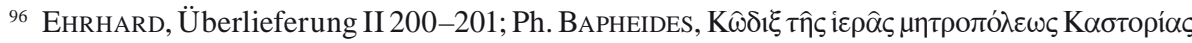

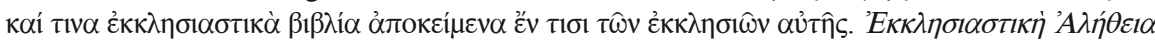
20 (1900) 143; KotTer, Die Schriften des Johannes von Damaskos V 6 (Nr. 24) und 166. - Textabfolge (Seitenangaben finden sich wiederum nur dort, wo sie mit Sicherheit der Sekundärliteratur entnommen werden konnten): Theodoros Studites, In nativitatem B. M. V., (p. 26-60) (Ps.-)Ioannes von Damaskos, Oratio in nativitatem sanctae Dei genitricis Mariae, Andreas von Kreta, Homilia I in nativitatem B. M. V., Andreas von Kreta, Homilia II in nativitatem B. M. V., Andreas von Kreta, Homilia III in nativitatem B. M. V.

${ }^{97}$ Ehrhard, Überlieferung III 473-475 (vgl. auch S. 480-482 unter der Sigle K); AthenA-

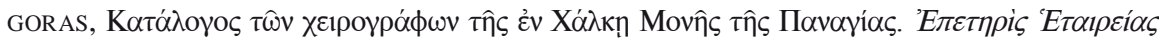

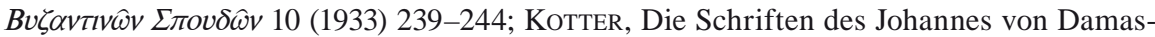
kos V 21 (Nr. 164) und 159; zur Datierung des Codex vgl. den Hinweis bei EhrHARD, a. O. 473, Anm. 2, daß die chronologischen Angaben zur Entstehungszeit (ed. Athenagoras, a. O. 243) auf f. $462^{v}$ von anderer Hand eingetragen sind; vgl. dazu auch die rezente Beschreibung des Chalcensis bei M. Kouroupou-P. GÉHIN, Catalogue des manuscrits conservés dans la Bibliothèque du Patriarcat EEcuménique. Les manuscrits du monastère de la Panaghia de Chalki I. Notices descriptives. Turnhout 2008, 63-67 (für die liebenswürdige Übersendung von Photokopien zu Einzelheiten dieses neuen Katalogs unmittelbar nach der Drucklegung danken wir Herrn Dr. Paul Géhin). - Textabfolge: (f. $1^{\text {r}}-5^{\text {r }}$; gezählt als $\beta^{\prime}$; davor fragmentarisches Ende der pseudo-chrysostomischen Homilie In principium indictionis [GeERARD, Clavis II 571 (Nr. 4597)]) Andreas von Kreta, Homilia I in nativitatem B. M. V., (f. $5^{r}-9^{v}$ ) Andreas von Kreta, Homilia IV in nativitatem B. M. V., (f. 9v-14r) (Ps.-) Ioannes von Damaskos, Oratio in nativitatem sanctae Dei genitricis Mariae, (f. $14^{\mathrm{r}}-20^{\mathrm{r}}$ ) Andreas von Kreta, Homilia II in nativitatem B. M. V. (unter dem Namen des Ioannes von Damaskos), (f. $20^{\mathrm{r}}-24^{v}$ ) Andreas von Kreta, Homilia III in nativitatem B. M. V. (unter dem Namen des Ioannes von Damaskos), (f. 24v-29r) Photios, Homilia in nativitatem B. M. V., (f. 29r-31') Leon der Weise, Sermo in B. M. V. partum.

${ }^{98}$ Druckvorlage für die Ausgabe von Combefis (1644): vgl. unten, S. 36.

${ }^{99}$ Ehrhard, Überlieferung II 95-96; OMONT, Inventaire sommaire (wie in Anm. 50) I 153-154; Halkin, Manuscrits grecs de Paris (wie in Anm. 50) 77-78; KотTER, Die Schriften des Johannes von Damaskos V 36 (Nr. 286) und 159. - Textabfolge: (f. 73-77) Andreas von Kreta, Homilia I in nativitatem B. M. V., (f. $77^{\mathrm{r}}-82^{\mathrm{r}}$ ) (Ps.-)Ioannes von Damaskos, Oratio in nativitatem sanctae Dei genitricis Mariae, (f. $82^{\mathrm{v}}-87^{\mathrm{r}}$ ) Andreas von Kreta, Homilia IV in nativitatem B. M. V. (unter dem Namen des Ioannes von Damaskos), (f. 87-93v) Andreas von Kreta, Homilia II in nativitatem B. M. V. (unter dem Namen des Ioannes von Damaskos), (f. 93ㄴ $-98^{v}$ ) Andreas von Kreta, Homilia III in nativitatem B. M. V. (unter dem Namen des Ioannes von Damaskos). 
Gabriel Sumarupas, Protosynkellos der Großen Kirche von Konstantinopel) (f. $1^{\mathrm{r}}-10^{\mathrm{r}}$ und f. $\left.60^{\mathrm{r}}-72^{\mathrm{v}}\right)^{100}$, Cod. Berol. gr. 43 (Phillipps 1447, Meerm. 90) aus dem Jahre 1608 (ebenfalls von der Hand des Gabriel Sumarupas) (f. 37 ${ }^{\mathrm{r}}-40^{\mathrm{r}}$ und $\mathrm{f}$. $\left.47^{\mathrm{r}}-51^{\mathrm{r}}\right)^{101}$, Cod. Chalc. Panag. Kamariotisses 47 aus dem Jahre 1608/1609 (von der Hand eines $\delta \iota^{\delta} \alpha \dot{\alpha} \sigma \kappa \alpha \lambda$ os Anthimos) (f. $13^{\mathrm{v}}-20^{\mathrm{v}}$ und f. $\left.57^{\mathrm{r}}-65^{\mathrm{v}}\right)^{102}$.

Zur Gruppe der Codices mit Einschüben anderer (scil. auf den 8. September bezüglicher) Texte zwischen der Homilia I und der Homilia III in nativitatem B. $M$. V. des Andreas von Kreta gehören auch folgende Handschriften, die allerdings die Abfolge der beiden Homilien invertieren, d. h. die Homilia III vor der Homilia I bringen: Cod. Athen. Ethn. Bibl. 224 aus dem 14. Jahrhundert ${ }^{103}$, Cod. Cpl. Metochion Pan. Taphou 244 aus dem 14. Jahrhundert (f. $12^{r}-19^{r}$ und f.

${ }^{100}$ Ehrhard, Überlieferung II 205-208; R. Devreesse, Le fonds Coislin. Paris 1945, 253-255 (mit der Namensform „Soumacoupa“ [EHrhard, a. O. 205, Anm. 1, liest Sumarypas; vgl. auch a. O. 206, Anm. 1; die korrekte Schreibung verdanken wir einer freundlichen Überprüfung durch Christian Foerstel am Original]); HaLKIN, Manuscrits grecs de Paris (wie in Anm. 50) 265-266; KotтeR, Die Schriften des Johannes von Damaskos V 41 (Nr. 319) und 164. - Textabfolge: (f. $1^{\mathrm{r}}-10^{\mathrm{r}}$ ) Andreas von Kreta, Homilia I in nativitatem B. M. V., (f. $\left.10^{\vee}-18^{r}\right)$ Andreas von Kreta, Homilia IV in nativitatem B. M. V., (f. 18 $\left.{ }^{\mathrm{v}}-34^{\mathrm{r}}\right)$ Andreas von Kreta, Homilia II in nativitatem B. M. V., (f. 34v-47) (Ps.)Ioannes von Damaskos, Oratio in nativitatem sanctae Dei genitricis Mariae, (f. $47^{\mathrm{r}}-59^{v}$ ) Theodoros Studites, In nativitatem B. $M$. V. (unter dem Namen des Ioannes von Damaskos), (f. $60^{\mathrm{r}}-72^{\mathrm{v}}$ ) Andreas von Kreta, Homilia III in nativitatem B. M. V., (f. 72v-84') (Ps.)Epiphanios von Kypros, Homilia V in laudes B. M. V., (f. 84-92') Kyrillos von Alexandreia, Encomium in B. M. V., (f. 92-98v) Proklos von Konstantinopel, Homilia de laudibus B. M. V.

101 Ehrhard, Überlieferung II 206; VAn DE Vorst-Delehaye, Catalogus (wie in Anm. 79) 153; W. Studemund-L. Cohn, Verzeichnis der griechischen Handschriften der königlichen Bibliothek zu Berlin I (= Codices ex Bibliotheca Meermanniana, Phillippici graeci nunc Berolinenses) (Die Handschriftenverzeichnisse der königlichen Bibliothek zu Berlin

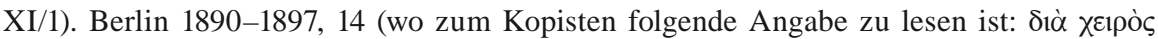

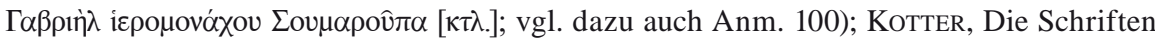
des Johannes von Damaskos V 18 (Nr. 140). - Textabfolge: (f. 37'-40r) Andreas von Kreta, Homilia I in nativitatem B. M. V., (f. $40^{\text {r-v }}$ ) Andreas von Kreta, Homilia IV in nativitatem B. M. V. (des. mut.), (f. $41^{\mathrm{r}}-47^{\mathrm{r}}$ ) Andreas von Kreta, Homilia II in nativitatem B. M. V., (f. $\left.47^{\mathrm{r}}-51^{\mathrm{r}}\right)$ Andreas von Kreta, Homilia III in nativitatem B. M. V.

102 Ehrhard, Überlieferung II 205; Athenagoras, Kató Kouroupou-GÉHin, Catalogue (wie in Anm. 97) 165-167; KotTER, Die Schriften des Johannes von Damaskos V 21 (Nr. 166) und 164. - Textabfolge: (f. $3^{r}-13^{v}$ ) Protevangelium Iacobi, (f. $13^{\mathrm{v}}-20^{\mathrm{v}}$ ) Andreas von Kreta, Homilia I in nativitatem B. M. V., (f. $21^{\mathrm{r}}-30^{\mathrm{v}}$ ) (Ps.-) Ioannes von Damaskos, Oratio in nativitatem sanctae Dei genitricis Mariae, (f. 30v-39v) Theodoros Studites, In nativitatem B. M. V. (unter dem Namen des Ioannes von Damaskos), (f. $40^{\mathrm{r}}-45^{\mathrm{v}}$ ) Andreas von Kreta, Homilia IV in nativitatem B. M. V. (zur korrekten Textabfolge [der Chalcensis spiegelt offensichtlich Blattvertauschungen in seiner direkten Vorlage wider] vgl. die Angaben bei Kouroupou-GÉHIN, a. O. 165), (f. 45-57') Andreas von Kreta, Homilia II in nativitatem B. M. V., (f. 57-65ำ Andreas von Kreta, Homilia III in nativitatem B. M. V. (unter dem Namen des Ioannes von Damaskos), (f. 65v-73v) (Ps.-)Epiphanios von Kypros, Homilia V in laudes B. M. V., (f. 74-79v) Kyrillos von Alexandreia, Encomium in B. M. V., (f. 79v-83v) Proklos von Konstantinopel, Homilia de laudibus B. M. V.

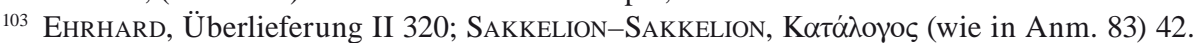
- Textabfolge (auf $3^{r}-18^{v}$; Ehrhard wertet diesen Blattbestand als „Zusatz“ zu dem Hauptblock des Athener Codex, der auf f. 20 [f. $19^{r-v}$ war ursprünglich unbeschrieben] mit der zum 1. September gehörenden Vita des Symeon Stylites des Älteren aus der Feder des Symeon Metaphrastes [BHG 1686] beginnt und der auf f. 35-38v die Homilia I in nativitatem B. $M$. V. des Andreas von Kreta enthält): Andreas von Kreta, Homilia IV in nativitatem B. M. V. (unter dem Namen des Ioannes von Damaskos), Andreas von Kreta, Homilia III in nativitatem B. M. V. (unter dem Namen des Ioannes von Damaskos), Andreas von Kreta, Homilia II in nativitatem B. M. V. (unter dem Namen des Ioannes von Damaskos); danach folgen als weiterer Bestand dieser „Ergänzung“ drei Texte für den 14. September. 
$\left.34^{\mathrm{r}}-40^{\mathrm{v}}\right)^{104}$, Cod. Athous Iber. 427 (Lambros 4547) aus dem Jahre 1560 (von der Hand eines Theokletos) $)^{105}$, Cod. Alexandr. Patr. 385 (173) aus dem 16. Jahrhundert (Hauptblock von der Hand eines Mönches Nikephoros) (p. 791-825 und p. 965-990) ${ }^{106}$, Cod. Chalc. Theol. Schol. 33 (29) aus dem 16. Jahrhundert ${ }^{107}$, Cod. Athous Dion. 168 (Lambros 3702) aus dem Jahre 1603/1604 (von der Hand eines Mönches Daniel) ${ }^{108}$, Cod. Athous Simonopetr. 44 (Lambros 1312) aus dem

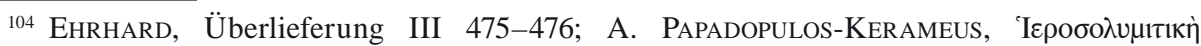

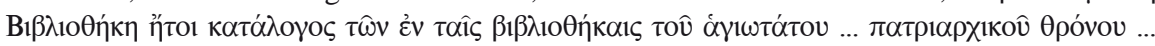

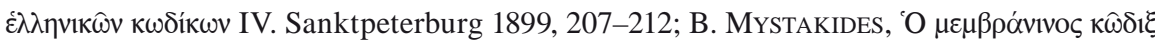

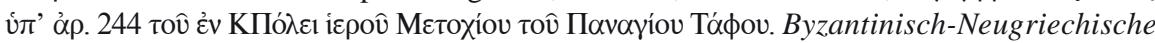
Jahrbücher 3 (1922) 351-359 (mit Datierung ins 12. Jahrhundert); KotTER, Die Schriften des Johannes von Damaskos V 23 (Nr. 180) und 164. - Textabfolge: (f. $3^{\mathrm{r}}-12^{\mathrm{r}}$ ) Protevange-

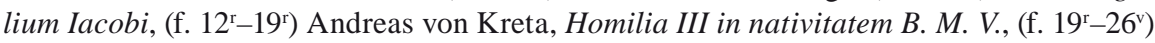
Theodoros Studites, In nativitatem B. M. V., (f. 27-34') (Ps.-)Ioannes von Damaskos, Oratio in nativitatem sanctae Dei genitricis Mariae, (f. $34^{\mathrm{r}}-40^{\mathrm{v}}$ ) Andreas von Kreta, Homilia I in nativitatem B. M. V., (f. 40-50') Andreas von Kreta, Homilia II in nativitatem B. M. V., (f. $50^{\mathrm{r}}-57^{\mathrm{r}}$ ) Andreas von Kreta, Homilia IV in nativitatem B. M. V.

105 Ehrhard, Überlieferung II 323-324 (mit Datierung in das Jahr 1554); LAmBros, Catalogue (wie in Anm. 95) II 145; KotTer, Die Schriften des Johannes von Damaskos V 11 (Nr. 71) und 165. - Textabfolge: Andreas von Kreta, Homilia III in nativitatem B. M. V., (f. 141 ${ }^{\mathrm{v}}$ $150^{r}$ ) (Ps.-)Ioannes von Damaskos, Oratio in nativitatem sanctae Dei genitricis Mariae, Theodoros Studites, In nativitatem B. M. V., Andreas von Kreta, Homilia IV in nativitatem B. M. V., Andreas von Kreta, Homilia I in nativitatem B. M. V.

106 H. Delehaye, Catalogus codicum hagiographicorum graecorum Bibliothecae Patriarchatus Alexandrini. Analecta Bollandiana 39 (1921) 351-353 (unter der Signatur Cod. 173); G.

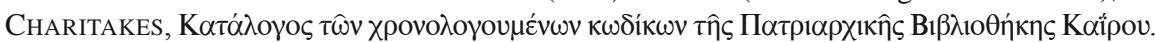

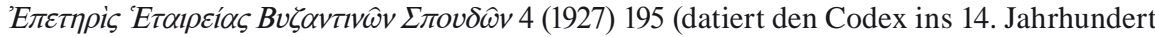
[die Subscriptio des Nikephoros auf p. 783 nennt nur Wochen- und Monatstag, kein Weltjahr

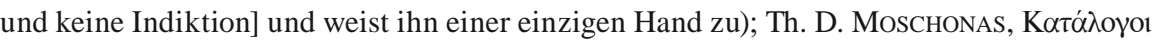

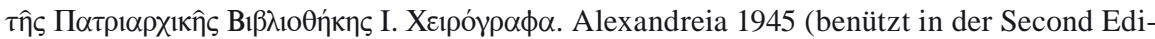
tion: Catalogue of Mss of the Patriarchal Library of Alexandreia [Studies and Documents XXVI]. Salt Lake City 1965), 223 (unter der Nummer 385; ohne Angaben zur Handtrennung). - Textabfolge: (p. 791 [also in jenem Teil, der nach Delehaye einer zweiten Hand zuzuweisen ist]-825) Andreas von Kreta, Homilia III in nativitatem B. M. V., (p. 826-868) Andreas von Kreta, Homilia I in exaltationem s. crucis (GEERARD, Clavis III 544 [Nr. 8179]; der Text gehört zum 14. September), (p. 869-900) Passio s. Euphemiae (BHG 619a; zum 16. September), (p. 915-956) Acta seu peregrinationes s. Iohannis Theologi (vgl. BHG 916; zum 26. September; des. mut.), (p. 965-990) Andreas von Kreta, Homilia I in nativitatem B. M. V.

107 EHrhard, Überlieferung III 492-495; H. DELEHAYE, Catalogus codicum hagiographicorum graecorum Bibliothecae Scholae Theologicae in Chalce insula. Analecta Bollandiana 44 (1926) 39-42 (unter der Signatur Theol. Schol. 33; vgl. dazu auch die Ergänzungen von H. Delehaye, Ad catalogum codicum hagiographicorum graecorum Bibliothecae Scholae Theologicae in Chalce insula supplementum. Analecta Bollandiana 46 [1928] 160, bzw. die Rezension von A. EHRHARD zum Katalog Delehayes, in: Byzantinische Zeitschrift 27 [1927] 126 [mit Hinweisen zu den zahlreichen herausgeschnittenen Blättern]); Ai. TsAKOPULOs

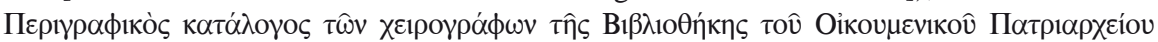

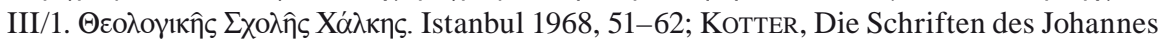
von Damaskos V 22 (Nr. 175) und 165. - Textabfolge: Theodoros Studites, In nativitatem B. M. V. (der Anfang fehlt), Andreas von Kreta, Homilia III in nativitatem B. M. V., (f. 11 ${ }^{\mathrm{r}}$ $19^{v}$ ) (Ps.-)Ioannes von Damaskos, Oratio in nativitatem sanctae Dei genitricis Mariae, Andreas von Kreta, Homilia IV in nativitatem B. M. V., (f. 28 ${ }^{\mathrm{r}}-29^{\mathrm{r}}$ ) Protevangelium Iacobi (nur der Anfang und das Ende), Andreas von Kreta, Homilia I in nativitatem B. M. V.

108 Ehrhard, Überlieferung III 492-495; LAmbros, Catalogue (wie in Anm. 95) I 353; KotTER, Die Schriften des Johannes von Damaskos V 9 (Nr. 54) und 165. - Textabfolge: Theodoros Studites, In nativitatem B. M. V., Andreas von Kreta, Homilia III in nativitatem B. M. V., (f. $42^{\mathrm{v}}-53^{\mathrm{r}}$ ) (Ps.-)Ioannes von Damaskos, Oratio in nativitatem sanctae Dei genitricis Mariae, Andreas von Kreta, Homilia IV in nativitatem B. M. V., (f. 82r) Protevangelium Iacobi (nur das Ende), Andreas von Kreta, Homilia I in nativitatem B. M. V. 
Jahre 1625/1626 (von der Hand eines Mönches Kyrillos; beim Bibliotheksbrand im Jahre 1891 vernichtet) (p. 34-49 und p. 101-113) ${ }^{109}$.

Während die hier angeführten jüngeren Handschriften neben den soeben festgehaltenen Ergebnissen zur Überlieferungsgemeinschaft der Homiliae I et III in nativitatem B. M. V. des Andreas von Kreta hinsichtlich der constitutio textus ${ }^{110}$ dieser Reden kaum zusätzliche Erkenntnisse erwarten lassen, so könnte sich unter textkritischen Gesichtspunkten ein alter Überlieferungszweig mit (griechischen) Marienhomilien in lateinischer Übersetzung als wichtig erweisen, den vor kurzem Árpád Orbán ediert und ausführlich untersucht hat ${ }^{111}$. Es handelt sich hiebei um einen in Oberitalien in der zweiten Hälfte des 9. Jahrhunderts entstandenen Codex, der aus seiner späteren Bibliotheksheimat Reichenau schließlich an die Großherzogliche Badische Hof- und Landesbibliothek (heute Badische Landesbibliothek) zu Karlsruhe gelangte, wo er unter der Signatur Aug. perg. 80 aufbewahrt wird ${ }^{112}$; die dort enthaltene Sammlung patristischer griechischer Texte zu Maria wurde wohl nicht lange vor der Entstehungszeit der Karlsruher Handschrift in Italien (vielleicht aus mehreren griechischen Vorlagen ${ }^{113}$ ) ins Lateinische übertragen, ohne daß sich der Übersetzer

${ }^{109}$ Lambros, Catalogue (wie in Anm. 95) I 117-118 (bei EhrHARD, Überlieferung, nicht er$\mathrm{fa} \mathrm{t}$ ); KotтER, Die Schriften des Johannes von Damaskos V 17 (ohne eigene Numerierung). - Textabfolge: (p. 34-49) Andreas von Kreta, Homilia III in nativitatem B. M. V., (p. 49-65) (Ps.-)Ioannes von Damaskos, Oratio in nativitatem sanctae Dei genitricis Mariae (?) (sichere Identifizierung aufgrund der kursorischen Angaben im Katalog bei Lambros nicht möglich), (p. 66-83) Andreas von Kreta, Homilia IV in nativitatem B. M. V., (p. 83-101) Protevangelium Iacobi, (p. 101-113) Andreas von Kreta, Homilia I in nativitatem B. M. V.

${ }^{110}$ Ein Schluß, der freilich erst durch eine Kollation aller Andreas-Codices abgesichert werden könnte - eine höchst undankbare Aufgabe, die den zukünftigen Editoren der Homilien des Andreas von Kreta überlassen sei. - Zu unseren Vorschlägen hinsichtlich der Identifizierung der handschriftlichen Vorlagen der bisherigen Drucke der Homiliae in nativitatem B. M. V. vgl. unten, S. 36-38.

111 Sermones in dormitionem Mariae. Sermones patrum graecorum praesertim in dormitionem assumptionemque beatae Mariae virginis in Latinum translati ex codice Augiensi LXXX (saec. IX), cura et studio Á. P. Orbán (Corpus Christianorum, Continuatio Mediaevalis CLIV). Turnhout 2000. - Ein Hinweis auf diesen Überlieferungszweig findet sich bereits bei GeErard, Clavis III 542 (Nr. 8170 und 8172; zu Andreas von Kreta). - Die bei GeErard, Clavis III 542, und bei GEERARD-NORET, Clavis Suppl. 470, genannten Übersetzungen ins Georgische, Arabische und Altkirchenslavische wurden von uns (mangels entsprechender sprachlicher Kompetenz) nicht berücksichtigt.

112 Hinsichtlich der Datierung und der Schriftheimat folgen wir den Angaben bei B. Bischoff, Katalog der festländischen Handschriften des neunten Jahrhunderts (mit Ausnahme der wisigotischen), Teil I: Aachen-Lambach (Bayerische Akademie der Wissenschaften, Veröffentlichungen der Kommission für die Herausgabe der mittelalterlichen Bibliothekskataloge Deutschlands und der Schweiz). Wiesbaden 1998, 338 (Nr. 1615); die noch im (ursprünglich im Jahre 1906 erschienenen) Katalog von Alfred HoLDER, Die Reichenauer Handschriften I. Die Pergamenthandschriften (Die Handschriften der Badischen Landesbibliothek in Karlsruhe 5). Wiesbaden ${ }^{2} 1970,220-222$ (vgl. auch 659-660), vertretene zeitliche Einordnung des Codex ins ausgehende 10. Jahrhundert ist damit hinfällig; vgl. auch W. Berschin, Greek Letters and the Latin Middle Ages. From Jerome to Nicholas of Cusa. Revised and expanded edition, translated by J. C. FraKES. Washington D. C. 1988, 154-155, bzw. Orbán, Sermones (wie in Anm. 111) VII und XII(ff.) (mit den Datierungsvorschlägen „Zweites Drittel des 9. Jahrhunderts“ bzw. „Ende IX. Jh.“). - Da der Codex, wie bereits angedeutet, auch in textkritischer Hinsicht wichtig ist, wird er (auf der Basis der Ausgabe von Orbán, ohne eigene Einsicht in die Handschrift) im Apparat unserer auf der Grundlage der Palimpsestfragmente im Cod. Vind. theol. gr. 160 erstellten Probeedition unter der Sigle £ angeführt.

113 OrbÁN, Sermones (wie in Anm. 111) VII, spricht von einem „wohlgeordneten Sammelwerk, hinter dem ein Plan steht“, was dafür spricht, daß die Textabfolge auf den Kompilator (bzw. 
(bzw. die Übersetzer ${ }^{114}$ ) mit Sicherheit identifizieren ließe(n) ${ }^{115}$. Der am Anfang durch den Verlust eines Quaternio verstümmelte Codex enthält an seinem nunmehrigen Beginn folgende, im vorliegenden Zusammenhang wichtige lateinische Übersetzungen ${ }^{116}$ : (f. $1^{\mathrm{r}}-2^{\mathrm{v}}$ ) Andreas von Kreta, Homilia I in nativitatem B. $M$. V. (mit verstümmeltem Incipit: ...] principalium personarum. propterea choros duco et festivitatem celebro et delibor et matri Verbi festivum offero munus ${ }^{117}$ ), (f. $3^{\mathrm{r}}-11^{\mathrm{v}}$ ) Andreas von Kreta, Homilia III in nativitatem B. M. V., (f. 11 $11^{\mathrm{v}}-17^{\mathrm{r}}$ ) Amphilochios von Ikonion, Oratio in occursum Domini ${ }^{118}$.

Um ein Zwischenrésumé zu ziehen: Für den ersten der beiden weiter oben gebrachten Rekonstruktionsvorschläge, für die Annahme, daß die 7. Lage unseres alten Codex ein Ternio war, in dem die Homilia III in nativitatem B. $M$. V. des Andreas von Kreta unmittelbar auf dessen Homilia I folgte, läßt sich unter den zum Vergleich herangezogenen ältesten griechischen Handschriften ein einziger Parallelfall namhaft machen, nämlich der Cod. Par. gr. $766^{119}$, der darüber hinaus das Recht für sich in Anspruch nehmen kann, neben unserem Palimpsest der einzige alte griechische Codex zu sein, in dem beide in die nähere Betrachtung zu ziehenden Marienhomilien (Nr. I und Nr. III) des Andreas von Kreta enthalten sind ${ }^{120}$. Mit einiger Wahrscheinlichkeit kann die alte lateinische Überlieferung im Cod. Aug. perg. 80 als zusätzliche Untermauerung der Ternio-Variante herangezogen werden, wenngleich hier der Vorbehalt zu berücksichtigen ist, daß der (oder die) lateinische(n) Übersetzer des 9. Jahrhunderts die beiden genannten Homilien des Andreas von Kreta aus zwei verschiedenen griechischen handschriftlichen Vorlagen bezogen haben könnte(n) ${ }^{121}$. Darüber hinaus läßt sich eine direkte Abfolge

auf den Übersetzer/auf die Übersetzer) zurückgeht; vgl. auch BERSCHIN, Greek Letters (wie in Anm. 112) 155.

114 Orbán, Sermones (wie in Anm. 111) 5-6, plädiert (auf der Basis der ins Lateinische übertragenenen Andreas-Texte) mit guten Gründen für mehr als einen Übersetzer; die Frage, ob in einem von ihnen Anastasius Bibliothecarius zu sehen ist, läßt sich nicht mit Sicherheit beantworten (vgl. ORBÁN, Sermones VII-VIII).

115 Sicher ist auf jeden Fall, daß die Karlsruher Handschrift nicht das Autograph (der Kompilation bzw. der Übersetzung[en]) darstellt: vgl. ORBÁN, Sermones (wie in Anm. 111) 5.

${ }^{116}$ Erfassung des Inhalts nach Orbán, Sermones (wie in Anm. 111) XII-XV.

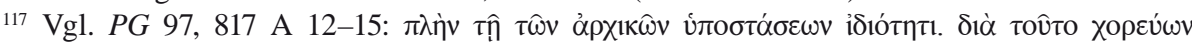

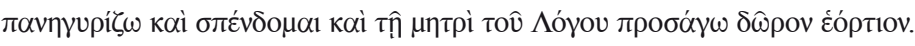

118 GeERARD, Clavis II 230 (Nr. 3232). - Auf die Anführung der weiteren im Cod. Aug. perg. 80 enthaltenen, aus dem Griechischen übersetzten lateinischen Texte wird hier verzichtet, weil wir mit der Hypapante-Homilie des Amphilochios von Ikonion (zum 2. Februar) die Reihe der Homilien zum 8. September bereits verlassen haben. - Für die Entstehungszeit der Übersetzung nicht unwesentlich ist das Faktum, daß der in Karlsruhe aufbewahrte Codex (auf f. $49^{v}-52^{v}$ ) die (im übrigen nur in lateinischer Fassung und nur hier erhaltene) Laus in celeberrimam dormitionem intemeratae dominae (sic) nostrae (sic) genetricis semperque virginis Mariae des Kosmas Bestitor enthält, dessen genaue ókuń freilich umstritten ist (zwischen 730 und 850? Vgl. Prosopographie der mittelbyzantinischen Zeit. Erste Abteilung [641-867], 2. Band: Georgios [\# 2183]-Leon [\# 4270]. Nach Vorarbeiten von F. Winkelmann erstellt von R.-J. Lilie-C. Ludwig-Th. Pratsch-I. Rochow. Berlin-New York 2000, 621 [\# 4125]; vgl. auch OrBÁN, Sermones [wie in Anm. 111] VII, Anm. 11); es folgen (f. 53r-69r) drei weitere (ebenfalls nur hier bezeugte) Homilien des Kosmas Bestitor zu Mariä Himmelfahrt.

119 Zum Parisinus vgl. die Hinweise oben, S. 14 mit Anm. 48-50.

${ }^{120}$ Alle anderen alten griechischen Handschriften bieten entweder nur die Homilia I oder nur die Homilia III.

121 Vgl. oben, S. 16 mit dem Hinweis, daß sich eine Überlieferungsgemeinschaft der Homiliae I et III in den griechischen codices vetusti neben unseren Palimpsestfragmenten nur in einem einzigen Fall, eben im Cod. Par. gr. 766, feststellen läßt. 
der Homilia I und der Homilia III in (mindestens) acht jüngeren Textzeugen belegen $^{122}$.

Die Entscheidung, ob bei der Rekonstruktion jenes Codex, dem unsere Palimpsestblätter entstammen, der Quaternio-Variante vor der Ternio-Version der Vorzug zu geben ist, muß von der Antwort auf die Frage ausgehen, wieviel Text auf den bei der Quaternio-Annahme anzusetzenden drei Blättern zwischen unseren Fragmenten $B^{v}$ und $C^{r}$ untergebracht gewesen sein könnte. Wie weiter oben gezeigt wurde ${ }^{123}$, enthielt ein Folium der ursprünglichen Handschrift rund 45 Migne-Zeilen (die weiterhin als behelfsmäßige Ausgangsbasis für derartige Rechenoperationen dienen müssen) ${ }^{124}$, was bei drei Blättern zwischen unseren Fragmenten $B^{v}$ und $C^{r}$ rund 135 Migne-Zeilen ergibt. Von dieser Zahl sind ca. 14 Migne-Zeilen (für das verlorene Ende von Homilia I) und ca. 32 Migne-Zeilen (für den ausgefallenen Begin von Homilia III) abzuziehen ${ }^{125}$. Der Textverlust zwischen dem seinerzeitigen Desinit von Homilia I und dem seinerzeitigen Incipit von Homilia III müßte somit rund 90 Migne-Zeilen (mit einer Toleranzgrenze von \pm 10 Migne-Zeilen) betragen haben.

Dieser Wert ist nun mit dem Umfang jener Homilien (scil. zu 8. September) in Verbindung zu bringen, die sich in den oben angeführten Codices in Überlieferungsgemeinschaft mit der Homilia I bzw. mit der Homilia III in nativitatem B. M. V. des Andreas von Kreta finden. Hier lassen sich in tabellarischer Zusammenfassung folgende Werte an Migne-Zeilen ${ }^{126}$ feststellen ${ }^{127}$ :

Andreas von Kreta, Homilia II in nativitatem B. M..$^{128}$ : 589 Migne-Zeilen Andreas von Kreta, Homilia IV in nativitatem B. M. V. ${ }^{129}$ : 472 Migne-Zeilen Basileios von Kaisareia, Quod Deus non est auctor malorum ${ }^{130}$ : 591 MigneZeilen

(Ps.-)Epiphanios von Kypros, Homilia V in laudes B. M. $V^{131}: 437$ Migne-Zeilen

122 Vgl. dazu die Übersicht oben, S. 16-17.

123 Vgl. oben, S. 10.

124 Vgl. unsere oben in Anm. 30 formulierten Vorbehalte.

125 Als weiterer Unsicherheitsfaktor kommt die Möglichkeit hinzu, daß sich nach dem Ende von Homilia I (auf dem Recto des ersten nach $\mathrm{f}$. $\mathrm{B}^{\mathrm{v}}$ ausgefallenen Blattes) ein unbeschrieben gebliebener Seitenrest befunden haben könnte und daß infolgedessen das Incipit eines neuen Textes erst auf dem Verso dieses ersten nach $\mathrm{f}$. $\mathrm{B}^{\mathrm{v}}$ in Verlust geratenen Blattes gestanden sein könnte; ebenso könnte die zumindest theoretisch anzusetzende Ausgestaltung dieses Incipit mit einem mehrere Zeilen einnehmenden Zierbalken (bzw. mit einer in leicht vergrößerten Buchstaben geschriebenen Überschrift) zu einer Verfälschung der rechnerischen Ausgangsbasis führen; beides (unbeschrieben gebliebene Seitenteile und Ausstattung eines Incipit mit einem Zierbalken) läßt sich etwa im Cod. Par. gr. 766 (auf f. 144v und auf f. 145; zu diesem Codex s. oben, S. 14 mit Anm. 48-50) nachweisen (aber nicht, um ein weiteres altes Vergleichsbeispiel zu zitieren, im Cod. Esc. $\Phi$-III-20, wo die Zierleisten jeweils eine einzige Zeile beanspruchen und die Überschriften in einer [aufrechten] Majuskel geschrieben wurden, die größenmäßig nicht ganz die Ausmaße der schrägovalen Majuskel der Homilientexte erreicht). - Diese Vorbehalte gelten natürlich auch für jenes verlorene Blatt, das einst den Beginn von Homilia III enthalten hat.

${ }^{126}$ Es gelten natürlich nach wie vor jene Kautelen, die schon mehrmals zur Brauchbarkeit von Rechenoperationen geäußert wurden, die auf der Grundlage von Zeilenzahlen in der „Patrologia graeca“ bei Migne durchgeführt werden.

127 Keine Berücksichtigung fanden natürlich jene Texte, die nachweislich erst nach der Entstehungszeit unseres Palimpsestes verfaßt wurden.

128 Vgl. Anm. 78 (hier und im folgenden wird auf jenen Abschnitt des vorliegenden Beitrags verwiesen, in dem der jeweilige Text zum ersten Mal Erwähnung findet).

129 Vgl. Anm. 79.

130 Vgl. Anm. 62.

131 Vgl. Anm. 91. 
Ioannes Chrysostomos, In nativitatem B. $M . V^{132}$ : ca. 55 Migne-Zeilen ${ }^{133}$ (Ps.-)Ioannes von Damaskos, Oratio in nativitatem sanctae Dei genitricis ${ }^{134}$ : 484 Migne-Zeilen

Kyrillos von Alexandreia, Encomium in B. M. . $^{135}$ : 284 Migne-Zeilen

Proklos von Konstantinopel, Homilia de laudibus B. M. $V^{136}: 255$ Migne-Zeilen

Protevangelium Iacobi ${ }^{137}$ : ca. 490 Migne-Zeilen ${ }^{138}$

Theodoros Studites, In nativitatem B. M. V.. ${ }^{139}$ : 466 Migne-Zeilen.

Aus dieser Übersicht ergibt sich, daß die Texte, die handschriftlich in Überlieferungsgemeinschaft mit den in unseren Fragmenten tradierten AndreasTexten bezeugt sind, einen viel zu großen Umfang haben, um (neben dem verlorenen Ende von Homilia I und dem fehlenden Beginn von Homilia III) in die Lücke von drei Blättern zu passen, deren Ausfall bei der Annahme eines ursprünglichen Quaternios zwischen $\mathrm{f}$. $\mathrm{B}^{\mathrm{v}}$ und $\mathrm{C}^{\mathrm{r}} \mathrm{zu}$ postulieren ist. Einzig und allein mit dem Chrysostomicum im Cod. Vat. gr. 2079, für das eine ungefähre Länge von 55 Migne-Zeilen berechnet werden konnte, ließe sich zur Not argumentieren, aber auch nur dann, wenn man von der Annahme ausgeht, daß jene Recto-Seite, die das Ende von Homilia I in B. M. V. des Andreas von Kreta enthielt, zu einem guten Teil unbeschrieben blieb (die rechte Kolumne dieser Seite müßte nur mit wenigen Zeilen gefüllt gewesen, der Rest leer geblieben sein), daß das Chrysostomicum dann auf der Verso-Seite dieses Blattes einsetzte und daß sich auch nach dem Desinit des Chrysostomicum leere Seitenteile befunden haben - aber auch dann geht die Rechnung nicht in einer wirklich befriedigenden Weise auf.

Zum Zwecke der Vollständigkeit: Auch bei der (grundsätzlich zwar nicht auszuschließenden, aber nicht sehr wahrscheinlichen) Annahme, daß jene Lage des alten Codex, dem unsere Palimpsestfragmente entstammen, ein Quinio gewesen $\operatorname{sei}^{140}$, läßt sich keiner der soeben genannten Texte umfangmäßig in den Blattverlust zwischen $\mathrm{f}$. $\mathrm{B}^{\mathrm{v}}$ und $\mathrm{C}^{\mathrm{r}}$ einbauen: Auf fünf Folien dieser alten Handschrift könnten zwischen dem Desinit der Homilia I und dem Incipit von Homilia III rund 180 Migne-Zeilen (mit einer Toleranzgrenze von \pm 15 MigneZeilen) untergebracht gewesen sein, doch ist auch dieser Wert für jeden der oben genannten zehn Texte viel zu gering.

Als Fazit bleibt somit nach einer Überprüfung der Ternio- bzw. Quaternio(und sicherheitshalber auch der Quinio-)Lösung im Grunde nur ein non liquet:

\footnotetext{
132 Vgl. Anm. 42.

${ }^{133}$ Da für diese (bis heute unedierte) Homilie kein Rückgriff auf Migne-Zeilen möglich war, wurde ihr Umfang wie folgt berechnet: Der Text nimmt im Cod. Vat. gr. 2079 (f. $120^{\mathrm{r}}, 5$. Z. v. u. $-121^{v}$, Seitenende) ein wenig mehr als drei Seiten ein; zieht man dazu als Vergleich die Werte heran, die sich für die Homilia I in nativitatem B. M. V. des Andreas von Kreta in dieser Handschrift feststellen lassen, so ergeben sich pro Seite rund 16 bis 18 Migne-Zeilen. Daraus folgt, daß man für das Chrysostomicum $3 \times 16 / 18=48 / 54+5$ Migne-Zeilen (vgl. schon oben, Anm. 43) ansetzen muß.

134 Vgl. Anm. 52.

135 Vgl. Anm. 91.

${ }^{136}$ Vgl. Anm. 82.

137 Vgl. Anm. 29.

${ }^{138}$ Wert geschätzt auf der Grundlage eines Vergleichs des Umfangs der Homilia I des Andreas von Kreta im Cod. Vat. gr. 455 (f. 208 $-213^{r}=357$ Migne-Zeilen) zum Umfang des Protevangelium Iacobi in der nämlichen Handschrift (f. 201'-208').

139 Vgl. Anm. 46.

${ }^{140}$ Vgl. oben, Anm. 34.
} 
Die Annahme eines Ternio löst zwar die Frage des Umfangs des zwischen $\mathrm{B}^{\mathrm{v}}$ und $C^{r}$ eingetretenen Blattverlustes relativ glatt, ist aber mit dem Nachteil behaftet, daß dann eine doch ziemlich auffällige (freilich nicht auszuschließende) Anomalie in der (vermuteten) Lagenzusammensetzung des „Muttercodex“ unserer Fragmente postuliert werden müßte. Diese Gefahr besteht bei der Quaternio-Variante zwar nicht, die dafür das so gut wie unlösbare Problem aufwirft, welcher Text zwischen dem Ende der Homilia I und dem Beginn der Homilia III in nativitatem B. M. V. des Andreas von Kreta untergebracht gewesen sein könnte - es könnte ja auch eine Marienhomilie gewesen sein, die heute nicht mehr erhalten ist (und deren Umfang füglich nur in Form eines Zirkelschlusses zu berechnen wäre); und schließlich darf auch noch die Möglichkeit nicht aus den Augen verloren werden, daß es von den zum Vergleich herangezogenen Texten, die in Überlieferungsgemeinschaft mit der Homilia I bzw. der Homilia III auftreten, heute nicht mehr (oder noch nicht ...) nachweisbare Rezensionen (mit einem von der sonstigen Tradition bzw. von dem Druck in der „Patrologia graeca“ abweichenden Umfang) gegeben haben könnte. Das sind der Unsicherheitsfaktoren einfach zu viele.

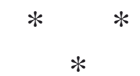

\section{TRANSKRIPTION}

Als erster Schritt bei der nun in Angriff zu nehmenden textkritischen Untersuchung der Andreas-Fragmente im Cod. Vind. theol. gr. 160 sei eine die äußerlichen Aspekte des Palimpsestes (Aufteilung auf Blätter, Seiten, Kolumnen und Zeilen ${ }^{141}$ ) möglichst getreu widerspiegelnde (den traditionellen Regeln einer diplomatischen Transkription ${ }^{142}$ folgende) Wiedergabe des Textes in Majuskelbuchstaben vorgelegt ${ }^{143}$, wobei auch ebenso möglichst getreu auf die (wie es scheint: stets von erster Hand stammende) Setzung von Spiritus, Akzenten, Tremata und Elisionszeichen im „Original“ Rücksicht genommen wird (natürlich mit dem Vorbehalt, daß sich in unserer Transkription nur die einigermaßen sicher lesbaren Hauch- und Betonungszeichen [etc.] finden) ${ }^{144}$. - Analoges gilt

${ }^{141}$ Vgl. besonders den Sonderfall in $C^{v}$ II, wo eine die sonstige Zeilenzahl (29) übersteigende Halbzeile (die in ihrer äußeren Form an die erst weitaus später anzutreffende Anbringung von Reklamanten erinnert) rechtsbündig unter Z. 29 angebracht wurde.

142 Etwa mit der üblichen Unterpunktung schlecht lesbarer Buchstaben. - Keine größenmäßig entsprechende graphische Berücksichtigung konnten bei der Transkription die wenigen, jeweils am Beginn einer Zeile stehenden „Initialen“ finden, die in unseren Fragmenten in sieben Fällen auftreten $\left(\mathrm{A}^{\mathrm{v}} \mathrm{II}^{5}\right.$ [Ny], $\mathrm{B}^{\mathrm{r}} \mathrm{II}^{5}$ [Epsilon], $\mathrm{B}^{\mathrm{r}} \mathrm{II}^{18}$ [Alpha], $\mathrm{B}^{\mathrm{r}} \mathrm{II}^{27}$ [Epsilon], B $\mathrm{B}^{\mathrm{v}} \mathrm{II}^{2}$ [Tau], $\mathrm{C}^{\mathrm{r}} \mathrm{II}^{26}$ [Ny], $\mathrm{C}^{\mathrm{v}} \mathrm{I}^{10}$ [Sigma]); es handelt sich bei ihnen im Grunde nur um vergrößerte, paläographisch in so gut wie keiner Weise bemerkenswerte Anfangsbuchstaben. Da es sich bei ihnen allerdings offensichtlich um „Lesehilfen“ (zur ungefähren Markierung eines größeren Sinneinschnittes) handelt, werden sie in unserer Transkription durch Fettdruck und, so wie in den Fragmenten selbst, durch eine Überschreitung des linken Randes des jeweiligen Schriftblocks hervorgehoben.

${ }^{143}$ Um keine Mißverständnisse aufkommen zu lassen: Alle folgenden Hinweise wollen keine in unseren Palimpsestfragmenten auftretenden „Sonderfälle“ dokumentieren: Wir stehen hier vor einer Art Norm, die auch in anderen, in etwa gleichzeitigen griechischen Majuskel(und natürlich auch - mutatis mutandis - in gleichzeitigen Minuskel-)codices zu beobachten ist.

${ }^{144}$ Das Fehlen eines Spiritus oder eines Akzentes an einer Stelle, an der die Setzung nach den Regeln der griechischen Sprache erforderlich wäre, besagt daher nicht, daß hier nicht doch 
auch für die Interpunktion (die in unsere Transkription vor allem deswegen aufgenommen wurde, weil die jüngste Forschung mit Nachdruck auf die Notwendigkeit, bei byzantinischen Texten auch dieses Detail zu beachten, hingewiesen hat ${ }^{145}$ ). Es hat den Anschein, daß der Kopist bei der Niederschrift des Andreas-Textes drei Arten von Satzzeichen verwendet hat: einen Punkt ${ }^{146}$ auf der Grundlinie (möglicherweise zur Kennzeichnung von schwächeren Einschnitten), einen Punkt in mittlerer Höhe und einen (offensichtlich einen stärkeren Einschnitt markierenden) hochgestellten Punkt (wobei manchmal eine korrekte Unterscheidung zwischen hochgestelltem und mittlerem Punkt kaum möglich ist $\left.{ }^{147}\right)^{148}$. - Die im Original vorhandenen Kürzungen (vor allem von nomina sacra ${ }^{149}$ ) werden in runden Klammern aufgelöst, wobei für den ergänzten Text ein kleinerer Schriftgrad zur Anwendung kommt ${ }^{150}$. - Die wenigen von erster Hand (wohl in scribendo) supraskribierten Buchstaben werden in

im „Urcodex“ ursprünglich ein Spiritus oder ein Akzent gestanden wäre, der dann durch die Palimpsestierung unlesbar gemacht wurde oder heute von der oberen Schrift überdeckt ist; ob hier durch mögliche Fortschritte in der digitalen Photographiertechnik in der Zukunft aussagekräftigere Resultate erzielt werden könnten, läßt sich nicht mit Sicherheit sagen (diese Feststellung gilt mutatis mutandis auch für alle folgenden paläographischen Beobachtungen). Akzente, die vom Kopisten in scribendo nicht über dem „richtigen“ Buchstaben gesetzt wurden, sondern über dem folgenden Buchstaben stehen, wurden von uns stillschweigend normalisiert; hingegen wurde in jenen (wenigen) Fällen, in denen in unserem Palimpsest ein „falscher“ Akzent (z. B. Akut anstelle eines Zirkumflexes) oder ein „falscher“ Spiritus verwendet wurde, nicht „normalisierend“ eingegriffen. Bei zwischen den Buchstaben der Diphthonge „schwebenden“ Spiritus und Akzenten wurde stets die „klassische“ Form der Setzung angewendet; nur bei den verschiedenen Formen von vióc, wo der Spiritus so gut wie ausnahmslos über dem Ypsilon steht, wurde diese „unkorrekte“ Form in der Transkription beibehalten. - Die wenigen Tremata stehen vornehmlich über Iota als Anfangsbuchstabe eines Wortes (ohne, wie es scheint, von einem Spiritus begleitet zu sein).

145 Vgl. D. R. Reinsch, Stixis und Hören; Referat auf dem VI e Colloque International de Paléographie Grecque, Drama, 21.-27. September 2003 (Kongreßakten im Druck; wir danken Herrn Kollegen Diether Roderich Reinsch herzlichst für die Zurverfügungstellung eines Manuskripts seines Beitrags und verweisen für die ältere Literatur zu dieser Frage auf die reiche Dokumentation in den Fußnoten seines im Druck befindlichen Referats).

${ }^{146}$ Dieser Punkt kann in Einzelfällen auch den Eindruck eines Beistrichs machen, ohne daß vom Text her eine sinngemäße Differenzierung zwischen „Punkt“ und „Beistrich“ möglich wäre; deswegen wird dieses Detail in unserer Transkription nicht berücksichtigt.

${ }^{147}$ Unsere Transkription läuft daher in Einzelfällen Gefahr, eine Ad-libitum-Entscheidung zu sein, doch ist es aufgrund der unserer Studie beigegebenen Tafeln jederzeit möglich, unsere Vorschläge im Detail zu überprüfen und gegebenenfalls zu revidieren (vgl. auch den „Sondertafelteil I“ zur Teildokumentation der Satzzeichen). - Bei schlecht lesbaren Interpunktionen wurde dieses Faktum, analog zu den schlecht lesbaren Buchstaben, durch Unterpunktung ausgewiesen; eine ebenfalls den Regeln der diplomatischen Transkription entsprechende Vorgangsweise wurde auch bei heute nicht mehr sichtbaren, aber in ihrer einstigen Existenz durch einen größeren Abstand zum folgenden Wort mit Sicherheit zu postulierenden Interpunktionen eingeschlagen: Hier finden eckige Klammern Anwendung.

${ }^{148}$ Ein absoluter Einzelfall ist ein hochgestellter, schräg nach rechts oben verlaufender pfeilförmiger Strich bei EI TI $\Sigma\left(B^{r}{ }^{26}\right)$ - vielleicht eine Lesehilfe bei einem stärkeren Sinneinschnitt (oder sollte es sich hier nur um einen besonders üppig ausgestalteten Akut handeln? Vgl. dazu die Gestaltung der analogen Wortfolge in $\mathrm{B}^{\mathrm{r}} \mathrm{II}^{1^{10}}$ ).

${ }^{149}$ Neben den Abbreviationen der nomina sacra werden in unserem Text nur zweimal $\kappa(\alpha i ́)\left(A^{v}\right.$ $\mathrm{I}^{16}$ und $\left.\mathrm{B}^{\mathrm{v}} \mathrm{I}^{3}\right)$ und einmal $\tau(\dot{\alpha} \varsigma)\left(\mathrm{C}^{\mathrm{r}} \mathrm{I}^{10}\right)$ gekürzt.

${ }^{150}$ Im Falle der nomina sacra bemüht sich unsere Transkription, die offensichtlich durchgehend gesetzten Kürzungsstriche nach Möglichkeit nachzuahmen, wobei natürlich die genaue Position und die Länge des Kürzungsstriches nicht immer hundertprozentig exakt wiedergegeben werden können (man vergleiche die Ausschnitte auf dem eigens zu einer Teildokumentation der Kürzungen beigegebenen „Sondertafelteil II“ zu den Abbreviatio- 
der Transkription tale quale wiedergegeben ${ }^{151}$. - Nicht berücksichtigt wurde die bei alten Majuskelhandschriften übliche scriptura continua: Als Lesehilfe wurde gegen den Befund des Originals eine durchgehende Worttrennung vorgenommen. - Für alle weiteren Einzelheiten (z. B. zu jenen wenigen Fällen, in denen der Kopist des Andreas-Textes Korrekturen vorgenommen hat, oder zu itazistischen [und anderen] Fehlern) verweisen wir auf den kritischen Apparat (in einem Fall auch auf den Testimonienapparat ${ }^{152}$ ) unter unserer Probeedition $^{153}$ bzw. auf jenen Abschnitt des vorliegenden Beitrags, der den paläographischen Aspekten der Fragmente gewidmet ist ${ }^{154}$.

nen); dort, wo heute der Kürzungsstrich wegen der Palimpsestierung nicht mehr sichtbar ist, wird er zwischen kleinen eckigen Klammern gesetzt.

${ }^{151}$ Sie dienen in einigen Fällen zur Verbesserung $\left(v g l . \mathrm{B}^{\mathrm{r}} \mathrm{II}^{5}\right.$ und $\left.\mathrm{B}^{\mathrm{v}} \mathrm{II}^{9}\right)$; einmal $\left(\mathrm{A}^{\mathrm{r}} \mathrm{II}^{16}\right)$ wurde der hochgestellte Buchstabe aus Platzgründen am Zeilenrand über den die Zeile abschließenden Buchstaben gesetzt. Im Falle des hochgestellten Omega über dritten Omikron im

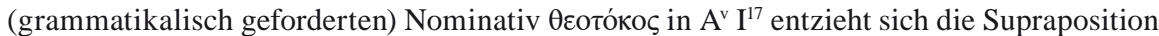
einer logischen Deutung.

${ }^{152}$ Vgl. zu C $\mathrm{C}^{\mathrm{v}} \mathrm{I}^{9}$, wo im Interkolumnium von erster Hand die Buchstaben AP (vielleicht ein Hinweis auf den Beginn des Zitats von Num. 36, 7-8) angebracht wurden.

153 Unten, S. 40-44.

${ }^{154}$ Unten, S. 45-46. 
$A^{\text {r }}\left(f .3^{\text {r }}+2^{v}\right): P G$ 97, 813 A 14-C 9

Col. I

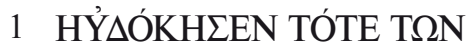

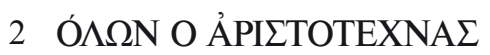

$3 \Theta(\overline{\mathrm{EO}}) \Sigma$. OÎON TINA ПANAPMO

4 NION KAI N়EOПAГĤ KÓ

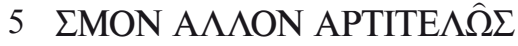

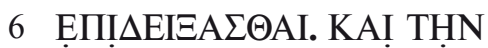

7 ЁГКАТАГКНЧАГАN ПА

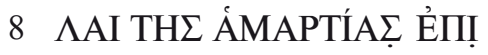

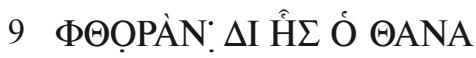

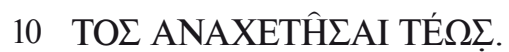

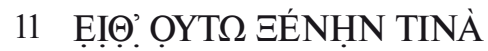

12 KAI A $\triangle \mathrm{OÝ} \Lambda \Omega \mathrm{TON}$ : KAI

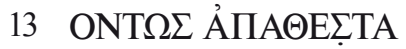

14 THN HMIN ПAPADEIEAI

15 Z $\Omega H N$. TOI $\Sigma$ ANAГEN

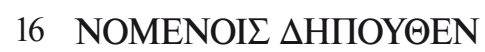

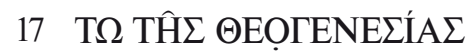

18 ВАПТÍ $\Sigma$ MATI' $\Pi \Omega \Sigma$

19 OYN É $\Delta$ EI TAYTHN EI $\Sigma$

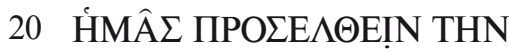

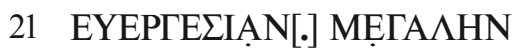

22 TE OY $\Sigma A N$ KAI ПAPA $\triangle$ O

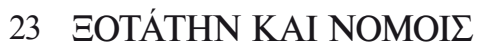

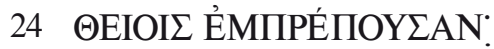

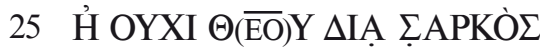

26 HMÎN. EПIФAINON

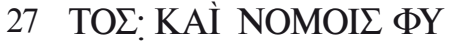

$28 \Sigma$ E $\Omega \Sigma$ EIKONTO $\Sigma$ KAİ

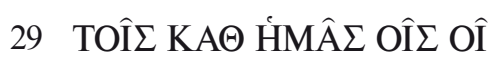

Col. II

$\Delta$ EN $\Lambda$ ОГОI $\Sigma$ ЕМПО $\Lambda$ ITEY

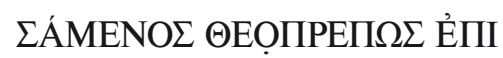

NEYONTOE TOYTO $\Delta$ È

TINA TPOחON EỊ $\Sigma$ ПEPA $\Sigma$ Ả

ГАГOITTO ÀN. H̉ ПАPЄENOY

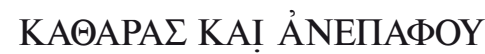

$\triangle$ IAKỌNOYMENH $\Sigma$ T $\Omega$

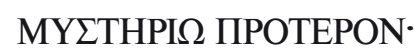

EÎTA KYOФOPOY

YIEPOY

NOMỌY ÝIIEPNIKŚN

TI TH $\Sigma$ ФÝ $\Sigma E \Omega \Sigma^{*}$ AÝ

TH $\Delta$ È TIL ÀN NỌOITO•

$\Pi \Lambda H N$ MONH $\Sigma$ ẺKEINH $\Sigma$

TH $\Sigma$ ПPO ПА $\Omega N$ ГENE

$\Omega$ N ẺK $\Lambda$ $\Lambda$ EГMÉNH $\Sigma \stackrel{\Omega}{T}$

ПANГENETI TH $\Sigma$ KTI

$\Sigma$ E $\Omega \Sigma^{*}$ AYTH ELTIN H

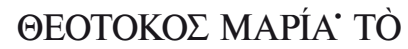

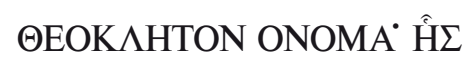

EK NI $\Delta$ YO

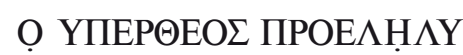

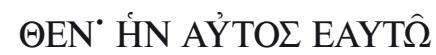

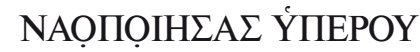

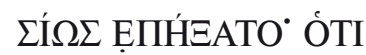

MH $\triangle$ IEФ@EIPEN TH̀N

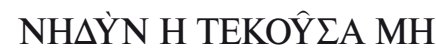

$\triangle$ E $\Sigma \Pi O P A \Sigma$ TÒ TEX@EN

$[\mathrm{E}] \Delta \mathrm{E} \Delta \mathrm{E} H \mathrm{HTO} \Theta(\overline{\mathrm{EO}}) \Sigma \Gamma$ $\Gamma$ ÀP ĤN. 
$A^{\text {v }}$ (f. $3^{\left.\text {v }+2^{r}\right): ~ P G ~ 97, ~ 813 ~ C ~ 9-816 ~ A ~} 5$

Col. I

1 EI KAI $\Sigma$ APKIK $\Omega \Sigma$ TENNH

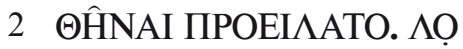

3 XÍA $\Sigma$ ÄNEY KAİ $\Omega$ ÍIN $\Omega N$

$4 \mathrm{X} \Omega \mathrm{PI} \Sigma\left[^{\circ}\right] \Omega \Sigma$ Ầ KAİ TA

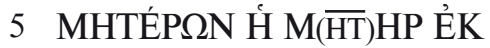

6 ФҮГЕІ. ПАРА $\triangle \mathrm{O} \Xi \Omega \Sigma$

7 ЁКТРЕ́ФОҮऽА ГА́ムАК

8 TI ỌN ẢNÁN $\triangle \mathrm{P} \Omega \Sigma$ ẺMA!

$9 \Delta$ OПOIH $\Sigma$ EN $\cdot$ KAİ Hं ПАP

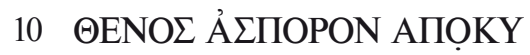

11 OY $\Sigma$ A ГENHMA• MENOI

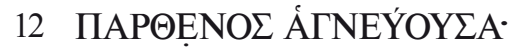

13 হ̦̂A KAİ METÀ TÒN

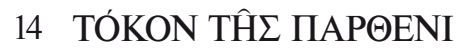

15 A $\Sigma$ TÀ $\Sigma$ HMAN $\triangle \mathrm{PA} \Phi$ Ẹ

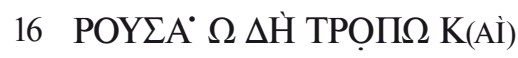

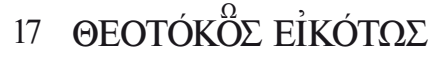

18 KHPYTTETAI KAI ПАP

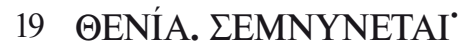

20 KAI ГẸNNH

21 NEITAI["] KAÌ $\Theta(\overline{\mathrm{EO}}) \Sigma$ ẢN $\Theta \mathrm{P} \Omega$

22 ПOI $\Sigma$ ÉNOYMENO $\Sigma \cdot$ Eİ

23 TA $\Sigma$ APKÌ ФANEPOY

$24 \operatorname{MENO} \Sigma$ TĤ $\Sigma$ OIKKIA $\Sigma$

$25 \Delta \mathrm{O} \Xi \mathrm{H} \Sigma$ XAPITAI TÒ

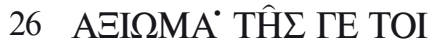

$27 \Pi \mathrm{\Pi P} \Omega \mathrm{TH} \Sigma$ ẺKEÍNH $\Sigma$ ẢPA $\Sigma$.

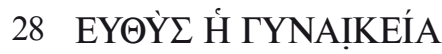

$29 \Phi Y \Sigma I \Sigma T H N N \Delta I O ́ P \Theta \Omega$
Col. II

$\Sigma$ IN $\Delta$ EXETAI. $\Omega \Sigma \Pi E P$

ENHPEATO TĤऽ ÁMAP

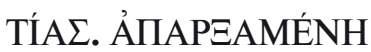

TĤ $\Sigma \Sigma(\overline{\Omega T H})$ PIA $\Sigma^{\bullet}$ HKEN TOI

NYN H'MÎN Ó $\Lambda$ ÓГO $\Sigma$ ЕП'AY

TO $\Lambda$ OIПON TÒ КЕФA

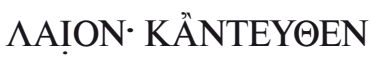

ẺГ̊̀ $\Sigma$ H́MEPON ПANHГY

PIక̣TH̀ $\Sigma$ KAI $\Lambda$ AMПPÒ $\Sigma$

É $\mathrm{TIÁT \Omega P} \mathrm{TĤ \Sigma} \mathrm{ÏEPA \Sigma}$

TAYTH $\Sigma$ ПAN $\Delta \mathrm{E} \Sigma \mathrm{IA} \Sigma$

ÝMÎN ПРОЕАH' $\Lambda Y \Theta A[$.

TH̀N ẸÝФPAऽIAN KỌI

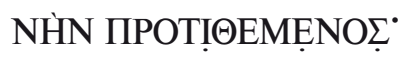

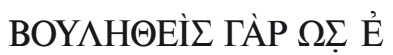

ФHN TOY ГÉNOY

TPSTH̀ $\Sigma$ NEAN ẢNTE

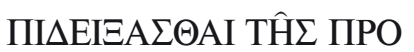

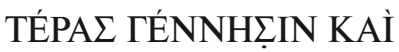

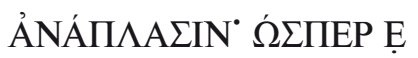

KEI ПРОTЕPỌN ẺK ПАP

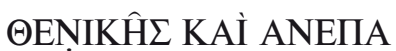

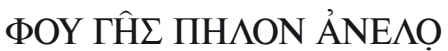

MENOE. TON ПIPß̂TON

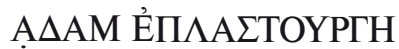

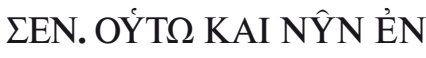

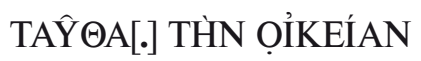

$\sum$ APK $\Omega \Sigma I N$ AÝTOYРГ $\Omega$ N.

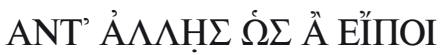




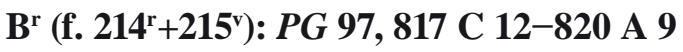

Col. I

1 ẢNEKAÍNHLEN $\Sigma Y \Gamma$

2 KPIMA` KAİ TAŶTA

3 MEN OÝT $\Omega \Sigma^{\cdot}$ HMEI

$4 \Delta$ È $\Omega$ TOŶ $\Theta(\overline{\mathrm{EO}}) \mathrm{Y} \Lambda \mathrm{AÓ} \Sigma^{*} . \Delta \mathrm{H}$

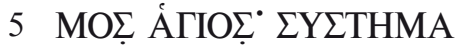

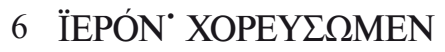

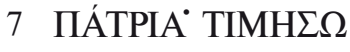

8 MEN TOŶ MY¿THPÍ

9 OY THN $\triangle$ ÝNAMIN $^{*}$

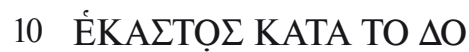

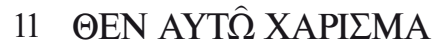

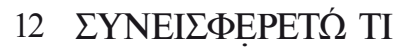

13 TĤ ПАNHГYPEI $\Delta \widehat{\Omega}$

14 PON EПÁEION["] OI ПA

15 TÉPES. THN EỶKAH

16 PIAN TOŶ ГÉNOY $\Sigma^{*}$

17 AÍ M( $\overline{\mathrm{HTE}}) \mathrm{PE} \Sigma$. THN EỶTE

18 KNÍAN: AI ¿TEIPAI.

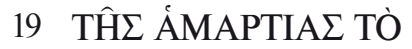

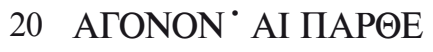

21 NOI TH̀N $\triangle \mathrm{II} \Lambda \Lambda H N$ Ả

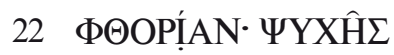

$23 \Lambda$ ЕГ $\Omega$ KAI $\Sigma \Omega_{M A T O}{ }^{*}$

24 ẠI ỴחỌ ZYTÒN TH̀N

25 EIENETH'N $\Sigma$ YMME

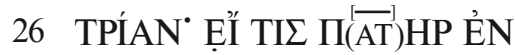

27 HMÎN* MIMEÎ $\Sigma \Theta \Omega$

28 TÒN TĤ $\Sigma$ ПAP@ENOY

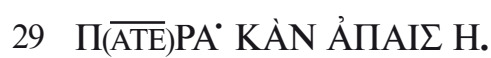

Col. II

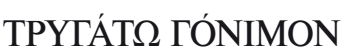

TH̀N EỶXHN. EK $\Phi I$

$\Lambda$ OEÉOY BÍOY TAY

THN KAPПOYMENO $\Sigma^{*}$

EI TI ${ }^{\Sigma}$ M( $\left.\overline{\mathrm{HT}}\right) \mathrm{HP} \Theta \mathrm{H} \Lambda$ ÁZOY

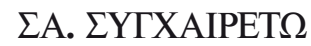

TH ẢNNA $\Theta H \Lambda A Z O Y$

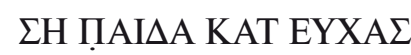

META $\Sigma T E I P \Omega \Sigma I N$ :

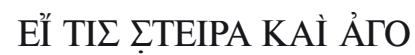

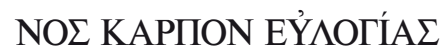

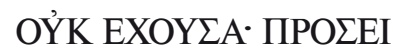

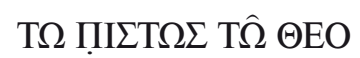

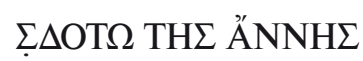

BุA $\Sigma T \widehat{T} \cdot$ KAI ẢПOKEI

PA $\Sigma \Theta \Omega$ TH̀N $\Sigma T E I ́ P \Omega$

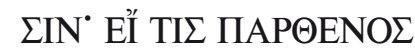

АГNEYOY $\Sigma$ A' ГENE $\Sigma \Theta \Omega$

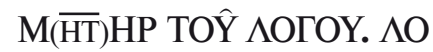

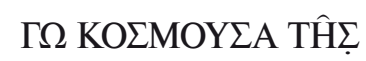

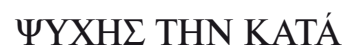

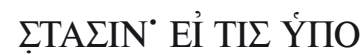

ZYTON. ПРОГАГЕT $\Omega$

$\mathrm{T} \Omega \Theta(\overline{\mathrm{E}}) \Omega$ KAPП $\Omega \mathrm{MA}$

$\Lambda$ OГIKON Ẻ $\Xi \hat{\Omega} N \Delta \mathrm{I}$ Ệ̉

XHิ $\Sigma$ ẺПOPÍ $\Sigma$ ATO[']

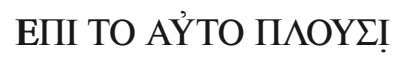

Ọ $\Sigma$ KAI חENH $\Sigma\left[{ }^{\circ}\right]$ NEANÍ

¿KOI KAI ПAP@ÉNOI- 
$B^{\mathrm{v}}$ (f. $\left.214^{\mathrm{v}}+215^{\mathrm{r}}\right):$ PG 97, 820 A 9-B 14

Col. I

1 ПРЕЕВÝTAI META NE

$2 \Omega T E ́ P \Omega N\left[{ }^{\circ}\right]$ ÏEPEI $\Sigma$ KAİ

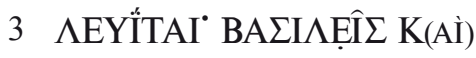

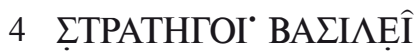

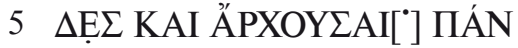

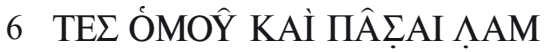

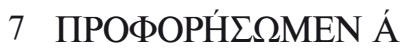

8 MẠ KAİ $\Delta \Omega P O Ф O P H$

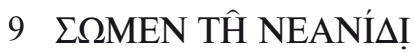

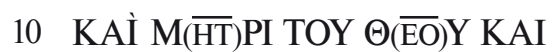

11 ПРОФНТІ $\Delta \mathrm{I}$ Ẹ $\hat{\mathrm{H}} \Sigma$ O

12 ПРОФН́TНऽ ÔN ЁГРА

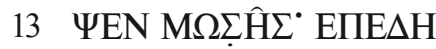

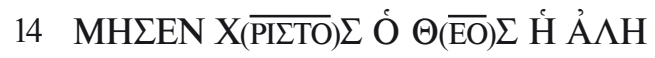

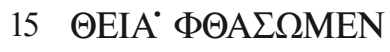

16 TOŶ NAOŶ TA ПРOПÝ

$17 \Lambda \mathrm{AIA}^{*} \Sigma \mathrm{YN} \triangle \mathrm{PAM} \Omega$

18 MEN TAI $\Sigma$ ПРОТTPEXOY

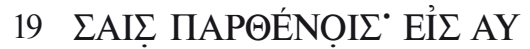

20 TÀ TA T $\Omega N$ AГI $\Omega N$

$21 \Sigma$ YNEI $\Sigma E ́ \Lambda \Theta Q M E N$ АГIA*

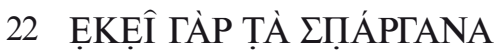

23 META ГÉNNHHূIN META

24 MAZÒN $^{*}$ META N̦H!̣IA

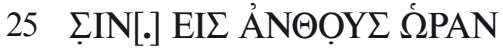

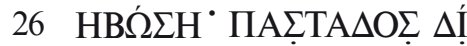

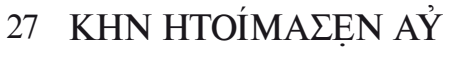

28 TH $\Theta(\overline{\mathrm{EO}}) \Sigma$ TO $\Theta P E \Pi T H P I O N[$.

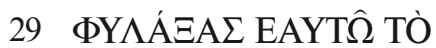

Col. II

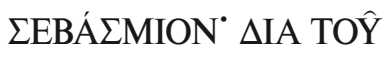

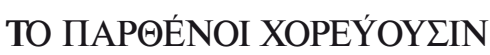

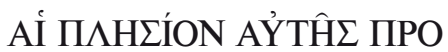

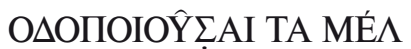

$\Lambda$ ONTA ' ẺNOEN Aİ TĤ $\Sigma$

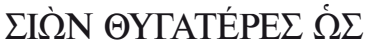

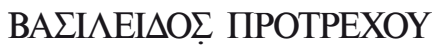

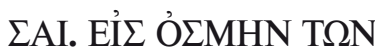

MYPO̊̊N AỶTH $\Sigma$ ПPOEЕÁP

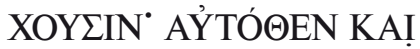

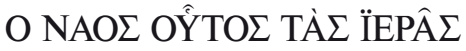

$\triangle$ IAПETÁ $\Sigma$ A $\Sigma$ ANEPPÍПH

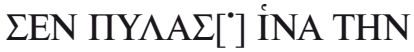

BALIAEION TOŶ ПIAN

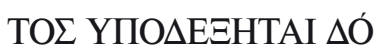

EAN` TOTE $\triangle$ H TOTE

TA T $\Omega N$ AГI $\Omega N$ ANE $\Omega$

ГNYNTO А̊ГIА: THN

ПANÁГION TOY ÁCÍOY

$\mathrm{M}(\overline{\mathrm{HTE}}) \mathrm{PA} \cdot \mathrm{EI} \Sigma \Omega \mathrm{T} \mathrm{T} \Omega \mathrm{N} A \Delta \mathrm{Y}$

T $\Omega$ N EKO $\Lambda \Pi \Omega \Sigma O M E N A^{*}$

ТРОФН $\triangle \mathrm{E}$ TẠÝTH $\Sigma$

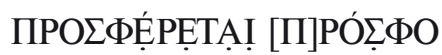

PO $\left[^{[}\right]$K KAİ TPEФEI TÉ $\Omega \Sigma$

ẢXEIP̣O $\Delta O ́ T \Omega \Sigma$ TH̀N

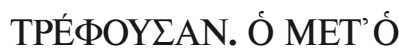

$\Lambda$ IГON T $\Omega$ AỶTH $\Sigma$ TPE

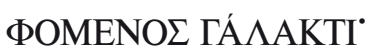

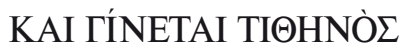


Col. I

1 MEN AỶPAI $\Sigma^{*} \Phi E P E ~ \Delta H$

2 KAİ AỶTḢN Eİ $\Sigma \Delta Y ́ N A$

3 MIN THN ПAPEENON

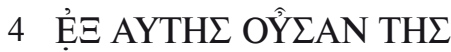

$5 \mathrm{PIZH} \Sigma \Delta \mathrm{A}(\overline{\mathrm{Y}}) \Delta \mathrm{A} \Pi \mathrm{A} \Delta \mathrm{EI} \Xi \Omega$

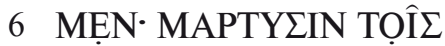

7 ЕŸАГГЕАІЕТАI $\Sigma$ XP $\Omega$

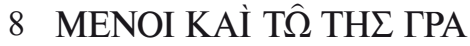

9 $\Phi \mathrm{H} \Sigma \mathrm{I} \Delta \mathrm{I} \Omega \mathrm{MATI}$

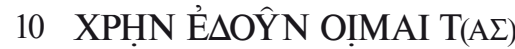

11 ẢПO TOŶ $\Delta \mathrm{A}(\overline{\mathrm{YI}}) \Delta$ ГENEA $\Sigma$

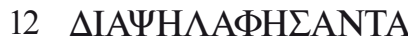

13 KATÀ THN TOY $\Lambda$ ỌY

14 KÂ KAÌ TOY MAT@AI

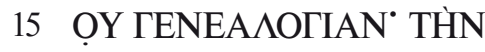

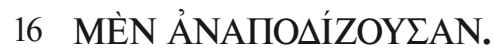

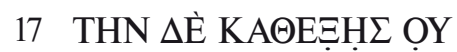

$18 \Delta$ EYOY $^{\prime} \mathrm{AN}^{*} \triangle \mathrm{IA} T \Omega \mathrm{N}$

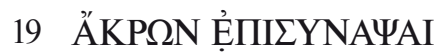

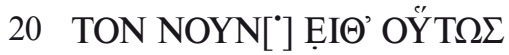

21 THN

22 H ПЕPI TOỲ $\Sigma$ TEKONTA $\Sigma$

23 TÒN Ï $\Omega \Sigma ̧ H \Phi ~ \Sigma Y N E X Y$

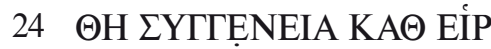

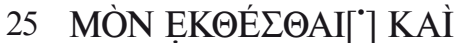

26 TỌN TPOחON KA@' ÓN KAİ

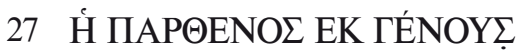

28 KATẠ́CETAI TOY $\Delta \mathrm{A}(\overline{\mathrm{YI}}) \Delta$

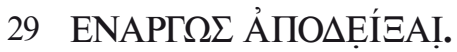

Col. II

KAI MHAÈN TŜN KAT AY

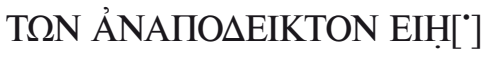
TOŶTON ГAP OÎ́ $\triangle A$ ПANTOહ̣

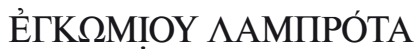

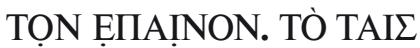

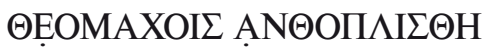
NAI $\Gamma \Lambda \Omega \Sigma \Sigma A I \Sigma:$ KAİ ПIAN ẢПОФРАЕАІ ГTOMA KA TAY TĤ $\Sigma$ ФPYA $\Sigma$ OMENỌN*AP

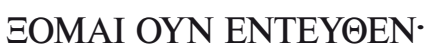
KAI MOI $\Sigma$ YГTIN $\Omega \Sigma K E$

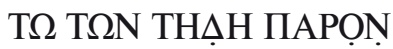

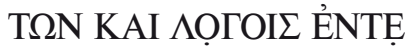
OPAMMEN $\Omega N$ EKALTO $\Sigma^{*}$

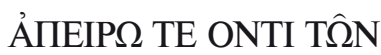

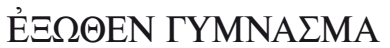
T $\Omega N[$.$] KAI TOY \triangle$ H XAPIN

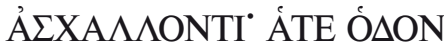
EIITOMMON OỶK EÝPILKON TI TOŶ $\triangle$ EONTO ФOPON` OM $\Omega \Sigma \mathrm{T} \widehat{\mathrm{N}} \Delta \mathrm{I} \Delta \mathrm{A}$

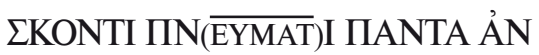

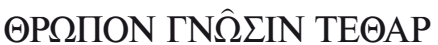
PHK $\Omega \Sigma$. ПРОГ TO ПРОКEÍ MEN ẢПOLYOMAI* NOMOE TOIГAP OŶN ẺKPA TEI TÒ ПA AAIÒN• MÍAN

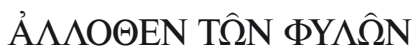

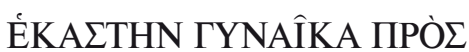


$C^{\mathrm{v}}\left(\right.$ f. $\left.4^{\mathrm{v}}+1^{\mathrm{r}}\right): P G$ 97, 845 B 12-D 7

Col. I

1 ГАMON АГЕ $\Theta A \mathrm{AI} \triangle \mathrm{YNA}$

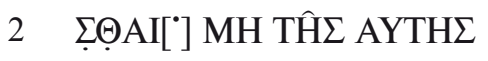

3 AYT $\Omega$ KOIN $\Omega N O Y \Sigma A N$

4 ФY $\Lambda \mathrm{H} \Sigma\left[{ }^{\circ}\right]$ OTI $\triangle \mathrm{E}$ TOY

5 TÓ Ẻ $2 T I N$ A $\Lambda H \Theta E \Sigma$. AY

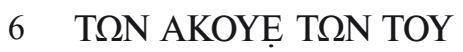

7 NÓMOY PHTQ̂N• EXXỌN

8 T $\Omega$ N ĖПI $\Lambda$ EEE $\Omega$ N OY

9 T $\Omega \Sigma\left[^{*}\right]$ ENETEI $A$ ATO M $\Omega$ APP

$10 \Sigma \mathrm{H} \Sigma$ TOI $\Sigma$ ÝIOÎ $\Sigma$ I $\Sigma$ PAH $\Lambda \Delta \mathrm{I} A$

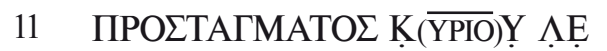

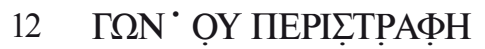

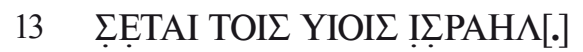

14 АПО ФYАН Е ЕІ

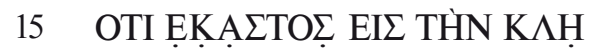

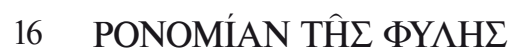

17 TĤ $\Sigma$ ПАТPIKH $\Sigma$ AYTOY

18 ПРО $К \mathrm{O} \Lambda \Lambda \mathrm{H} \Theta \mathrm{H} \Sigma \mathrm{ONTAI}$

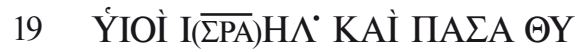

20 ГАТНР АГХІІТЕYОY

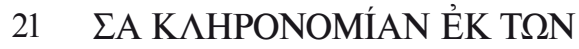

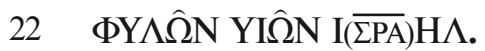

23 ENI TŜN EK TOY $\triangle$ HMOY

$24 \mathrm{TH} \Sigma \Phi \mathrm{Y} \Lambda \mathrm{H} \Sigma$ TOY $\Pi(\overline{\mathrm{AT}}) \mathrm{P}(\mathrm{O}) \Sigma \mathrm{AY}$

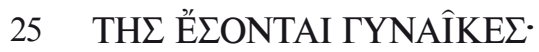

26 ÍNA АГXIక়TEÝOY IIN Ý

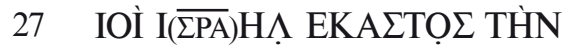

28 KАHPONOMIAN TH̀N ПA

29 TPIKH̀N AỶTOY• ON TPÓ
Col. II

ПIN $\Sigma$ YNETA $\Xi E N ~ K(\overline{\mathrm{YPIO}}) \Sigma \mathrm{T} \hat{\Omega}$

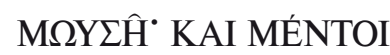

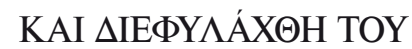
TO MEXPI KAIPOY ${ }^{*} \mathrm{E} \Omega \Sigma$ ГÀP H̉N@EI TA ÏOYAAÍ $\Omega \mathrm{N}^{`}$ KAI TA M $\Omega \Sigma$ AIKA $\triangle$ IIATÁГMATA HKMA ZEN. OỶ

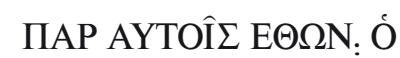
MH KATA @ELMON EKPÁ

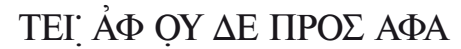

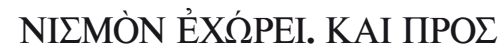
TOỶNANTION ÁПAN АПНYTOMÓ

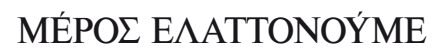
NA. OỶ KÉTI $\Lambda$ ÓГO $\Sigma$ АỶ TOI

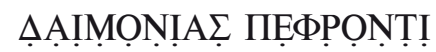

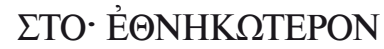
$\Pi \Omega \Sigma \Delta$ IAKEIMENOI $\Sigma$

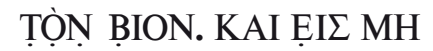

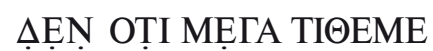
NOI TOŶ NOMOY EELIIZON ТО̣ $\Sigma^{*}$ Tล̂N ПРОФH

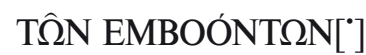
KAİ $\Delta \mathrm{IA} \Pi A \dot{\Sigma} \mathrm{H} \Sigma \Sigma \mathrm{XE} \Delta \mathrm{O} N$

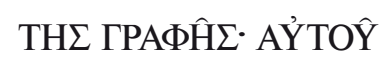
$\triangle$ IAMAPTYPOYMENOY TOY $\Theta(\overline{\mathrm{EO}}) \mathrm{Y}\left[{ }^{*}\right]$ 


\section{PROBEEDITION}

Um die in den Palimpsesten des Cod. Vind. theol. gr. 160 vorliegende Tradition der Homiliae I et III in nativitatem B. M. V. des Andreas von Kreta zumindest provisorisch in textkritischer Weise zu untersuchen und in Form einer (natürlich ebenfalls provisorischen) kritischen Edition darbieten zu können, wurden neben den oben ausgewiesenen älteren Codices ${ }^{155}$ auch alle vorhandenen Andreas-Editionen herangezogen (und nach Möglichkeit auf ihre handschriftliche Ausgangsbasis hin überprüft).

Die editio princeps der Homilia I in nativitatem B. M. V. des Andreas von Kreta verdanken wir François Combefis, der diesen Text im Jahre 1644 aus dem „MS. Reg. n. 275“ herausgegeben hat ${ }^{156}$, aus einer Handschrift, die nach den Pariser Signaturkonkordanzen ${ }^{157}$ mit dem heutigen Cod. Par. gr. 819 identifiziert werden kann.

Die zweite, von Combefis nach einem Pergamentcodex „ex eminentissimi Cardinalis Mazarini bibliotheca“" verbesserte Ausgabe dieser Homilie erschien im Jahre 1648 $8^{159}$; aufgrund der von Combefis zu seiner handschriftlichen Grundlage gebotenen Informationen ${ }^{160}$ ist dieser „codex Cardinalis Mazarini“ mit hoher Wahrscheinlichkeit mit dem heutigen Cod. Vat. Reg. gr. $15^{161} \mathrm{zu}$ identifizieren ${ }^{162}$.

155 Vgl. S. 13ff.

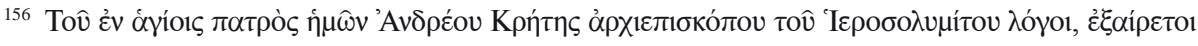

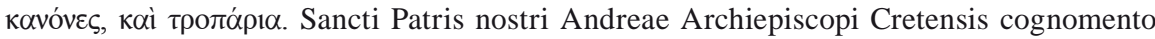
Hierosolymitani Orationes, selectique Canones, et Triodia. R. P. Francisci CombEFIS ... opera et studio, Latinè reddita, notisque illustrata, adiecto etiam peculiarum Andreae vocum copioso Indice, ac ceu Onomastico. Paris (Apud Simeonem Piget) 1644, 1-10 (in unserem Apparat: Comb ${ }^{\mathbf{1}}$ ); Nennung der handschriftlichen Grundlage: a. O. 1.

157 H. Omont, Anciens inventaires et catalogues de la Bibliothèque Nationale. Paris 1921, 53 und 82.

158 Zu ihm vgl. oben, S. 20 (mit Anm. 99). - Im Apparat unserer Probeedition: F (F wird im Apparat allerdings nur dann erwähnt, wenn der Text vom Druck Comb ${ }^{1}$ abweicht).

159 S. Patris Nostri Asterii Amaseae Episcopi, aliorumque plurium dissertissimorum ecclesiae graecae patrum ac Tractatorum lectae nouae eruditissimaeque, cum pari pietate Orationes et Homiliae: in Dominicas praesertim, sanctissimaeque Dei Genitricis solennitates. Opera et studio R. P. Francisci Combefis (= Graeco-Latinorum Patrum Bibliothecae novum auctarium, opera ac studio Francisci CombefIs). Paris (Sumptibus Antonii Bertier) 1648, 1289-1305 (in unserem Apparat: Comb²).

160 Sie finden sich in den unpaginierten Einleitungspartien, im Vorwort („Candido lectori“) („egregium MS membranaceum Codicem“) und im „Elenchus tomo hoc exegetico contentorum“; vgl. auch die „Notae“ am Ende der Edition der Andreas-Texte (a. O. 1363-1364).

161 Zu ihm vgl. oben, S. 19 (mit Anm. 94). - Im Apparat unserer Probeedition: R (R wird im Apparat ebenfalls nur dann erwähnt, wenn sein Text vom Druck Comb² abweicht).

162 Anders hingegen KotTer, Die Schriften des Johannes von Damaskos V 162 (in seiner Übersicht zur handschriftlichen Überlieferung des [Ps.-]Ioannes von Damaskos, Oratio in nativitatem sanctae Dei genitricis Mariae): Kotter sieht in dem von Combefis verwendeten „Mazarinus“ den Cod. Par. gr. 1171 (aus dem Besitze des Kardinals Mazarin: vgl. OMONT, Inventaire sommaire [wie in Anm. 50] I 234-235), und zwar auf der Grundlage eines Vergleichs der von Combefis (in seiner Edition des [Ps.-]Ioannes von Damaskos aus dem Jahre 1662 [vgl. dazu KotTer, Die Schriften des Johannes von Damaskos V 59]) ausgewiesenen Lesarten (scil. zu dem Pseudo-Damascenicum) mit der handschriftlichen Überlieferung. Kotters Überlegungen kranken freilich daran, daß er seine Suche nach Codices aus dem Besitze des Kardinals Mazarin ausschließlich auf Handschriften der Bibliothèque nationale Paris beschränkte (obwohl ihm an anderer Stelle [KоTTER, Die Schriften des Johannes von Damaskos V 49 (Nr. 383)] durchaus geläufig ist, daß auch der Cod. Vat. Reg. gr. 15 aus der Sammlung Mazarins stammt). - Unsere Entscheidung, den Cod. Vat. Reg. gr. 15 mit jenem Codex zu identifizieren, den Combefis für seine Andreas-Ausgabe aus dem Jahre 
Im Jahre 1779 übernahm Andrea Gallandi im 13. Band seiner „Bibliotheca veterum patrum“"163 den Andreas-Text der ersten Combefis-Ausgabe (1644) ${ }^{164}$, und aus Gallandi bezog Jean-Paul Migne den Text der Homilia I in nativitatem B. $M$. V. des Andreas von Kreta für seinen Druck im 97. Band der „Patrologia graeca“ $(1860)^{165}$. - Welche Handschriften Ioannes Patusas in seiner Ausgabe der Homilia I in nativitatem B. $M . V^{166}$ verwendet hat, ließ sich nicht feststellen.

Ein davon verschiedenes Schicksal nahm die Drucklegung der Homilia III in nativitatem B. M. V. des Andreas von Kreta. Hier verdanken wir die editio princeps Jacques de Billy, der diese Rede im Jahre 1577 in seiner Ausgabe der Werke des Ioannes von Damaskos aus einem Codex edierte, den er „eruditissimi viri Iacobi Cuiacij beneficio“ erhalten hatte ${ }^{167}$; diese Handschrift aus dem Besitze des berühmten Juristen Jacques Cujas wird von Bonifaz Kotter korrekt mit dem heutigen Cod. Par. Suppl. gr. 773 identifiziert ${ }^{168}$. - Direkt aus de Billy

1648 (und für seine Edition des [Ps.-]Ioannes von Damaskos aus dem Jahre 1662) heranzog, gründet sich auf folgende Beobachtungen: Zum ersten entsprechen die von Combefis zum Inhalt seiner Ausgabe des Jahres 1648 festgehaltenen Angaben („Elenchus ...“: s. oben, Anm. 160) ziemlich genau dem Cod. Vat. Reg. gr. 15, nicht aber dem Cod. Par. gr. 1171 (der die Homilia I in nativitatem B. M. V. des Andreas von Kreta nicht enthält!); zum zweiten läßt sich Combefis’ Feststellung, seine Vorlage sei zwar „plene probatus, sed non usquequaque integer“ (vgl. nochmals KoTTER, Die Schriften des Johannes von Damaskos V 162), ohne die geringsten Schwierigkeiten auf den (in Teilen verstümmelten) Cod. Vat. Reg. gr. 15, nicht aber auf den Cod. Par. gr. 1171 beziehen (Kotter ist sich dieser Problematik durchaus bewußt, zieht aber daraus keine Schlüsse).

163 Bibliotheca veterum patrum antiquorumque scriptorum ecclesiasticorum, postrema Lugdunensi longe locupletior atque accuratior, cura et studio Andreae GALLANDII, Bd. XIII. Venedig (Ex typographia Joannis Baptistae Albriti) 1779, 93-98.

164 In unserem kritischen Apparat werden die von Comb ${ }^{1}$ abweichenden Lesarten des Drucks bei Gallandi (meistens den Text verschlechternde Fehler) mit der Sigle Gall ausgewiesen.

165 Patrologiae cursus completus, accurante J.-P. Migne. Series graeca, Bd. 97. Paris 1860, 805-820. Vgl. dazu GEERARD, Clavis III 542 (Nr. 8170). - In unserem Apparat werden nur die Abweichungen des Druckes bei Migne vom Text bei Gallandi angegeben (und zwar mit der Sigle Mi).

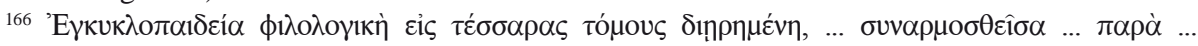

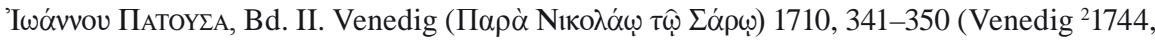

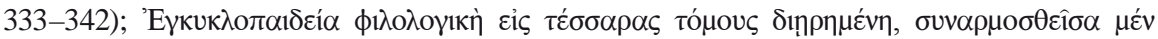

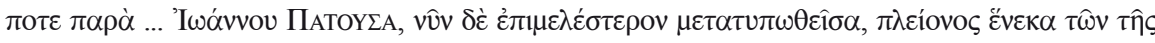

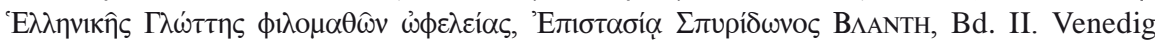

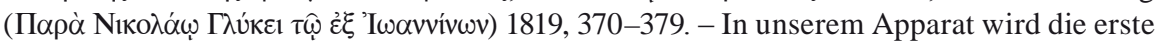
Auflage mit der Sigle Pat ausgewiesen, die Auflage des Jahres 1819 mit der Sigle PatBla (jedoch nur dann, wenn sie von der ersten Auflage abweicht).

167 Sancti Joannis Damasceni opera, multo quam unquam antehac auctiora, magnaque ex parte nunc de integro conversa ... per D. Jacobum Billium ... . Paris (Apud Guillielmum Chaudiere) 1577 , f. $380^{\vee}-385^{v}$ (in unserem Apparat: Bill); Nennung der handschriftlichen Grundlage: a. O. f. $357^{\text {r }}$.

168 Vgl. Kotter, Die Schriften des Johannes von Damaskos V 162 (zu Nr. 325); vgl. auch a. O. 41-42; s. auch DENS., Die Überlieferung der Pege Gnoseos des hl. Johannes von Damaskos (Studia Patristica et Byzantina 8). Ettal 1959, 58 (Nr. 501) und 228. Hauptargumente für die Identifikation der von de Billy herangezogenen Druckvorlage mit dem Cod. Par. Suppl. gr. 773 sind die Übereinstimmung der Lesarten seiner Edition mit der vom Parisinus gebotenen Textform und die Hinweise, die de Billy zu seiner handschriftlichen Grundlage bietet (a. O. f. 357): „... codicem, qui praeter has orationes Physicen etiam complectebatur“ („hae orationes“ sind folgende von de Billy edierte Texte: Ioannes von Damaskos, Homilia in transfigurationem Domini [im Parisinus erst an zweiter Stelle, von de Billy an den Beginn gerückt, um in seiner Ausgabe einen geschlossenen Block der Homilien auf Maria zu erzielen], [Ps.-]Ioannes von Damaskos, Oratio in nativitatem sanctae Dei genitricis, Andreas von Kreta, Homilia II und Homilia III in nativi- 
bezog Jean-Paul Migne den Text der Homilia III in nativitatem B. M. V. für seinen (nunmehr unter dem Namen des Andreas von Kreta erfolgenden) Druck im 97. Band der „Patrologia graeca“ (1860) ${ }^{169}$.

Bei der auf dieser Basis erarbeiteten Probeedition wurden folgende Prinzipien beachtet: Auch wenn in erster Linie die durch unser Palimpsest vertretene (mit $\Pi$ bezeichnete) Textform gehalten wurde, mußte doch an allen jenen Stellen, an denen die von uns in dem soeben skizzierten Umfang herangezogene Überlieferung einen in unseren Augen besseren Text bietet, „über dem Strich“ korrigierend eingegriffen werden ${ }^{170}$; um jedoch dem Leser stets eine konkrete Information über den Textzustand im Palimpsest zu bieten, wurden derartige Abweichungen sowohl im eigentlichen Text wie auch im kritischen Apparat durch Unterstreichung hervorgehoben; auf diese Weise soll dem Leser auch die Möglichkeit gegeben werden, unsere Entscheidung für oder gegen $\Pi$ zu überprüfen. Ansonsten versucht der Apparat, die Varianten bzw. die Fehllesungen in den zum Vergleich herangezogenen Codices (und in allen früheren Editionen $^{171}$ ) möglichst vollständig zu dokumentieren ${ }^{172}$.

tatem B. M. V. [beides unter dem Namen des Ioannes von Damaskos]; die „Physice“ entspricht den Kapiteln 67 und 68 der Dialectica des Ioannes von Damaskos [vgl. KoтteR, Überlieferung der Pege Gnoseos 228]). - Zur Handschrift selbst (in der sich unter anderem die Hände des Arnoldus Arlenius, des Bartolomeo Zanetti, des Ioannes Mauromates und des Petros Karneades nachweisen lassen: vgl. E. GamillscheG-D. HARLfinger, Repertorium der griechischen Kopisten 800-1600, 2. Teil: Handschriften aus den Bibliotheken Frankreichs und Nachträge zu den Bibliotheken Großbritanniens. A. Verzeichnis der Kopisten [Veröffentlichungen der Kommission für Byzantinistik III/2A]. Wien 1989, 39, 41, 98, 173; die uns in Photographien zur Verfügung stehenden f. 226 ${ }^{\mathrm{v}}-233^{\mathrm{r}}$ sind mit höchster Wahrscheinlichkeit einer Atelierhand zuzuweisen, die B. Mondrain, Copistes et collectionneurs de manuscrits grecs au milieu du XVI $\mathrm{I}^{\mathrm{e}}$ siècle: le cas de Johann Jakob Fugger d'Augsbourg. Byzantinische Zeitschrift 84-85 [1991-1992] 354-390 [vgl. besonders Abb. IIIb auf S. 388], als «occidental arrondi» bezeichnet hat; von Arlenius

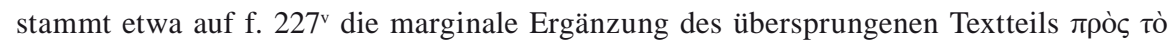

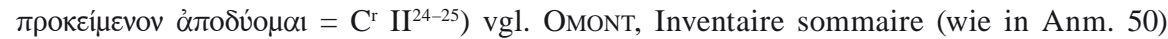
III 310, bzw. Halkin, Manuscrits grecs de Paris (wie in Anm. 50) 295; der Text der Homilia III findet sich, wie bereits gesagt, auf f. 226 ${ }^{\mathrm{v}}-233^{\mathrm{r}}$ unter dem Namen des Ioannes von Damaskos (Tô̂ aủrô̂ mit Bezug auf die Nennung auf f. 199'). - Im Apparat unserer Probeedition: S (S wird im Apparat nur dann erwähnt, wenn sein Text vom Druck bei de Billy abweicht).

169 Migne, Patrologia (wie in Anm. 165) 844-861. Vgl. dazu auch GeERARd, Clavis III 542 (Nr. 8172). - In unserem Apparat werden nur die Abweichungen des Druckes bei Migne vom Text bei de Billy angegeben (und zwar mit der Sigle Mi).

${ }^{170}$ Nach dem Prinzip eines in dubio pro reo wurde in Zweifelsfällen (bei denen eine endgültige Entscheidung nur nach einer erschöpfenden Erfassung der gesamten Überlieferung bzw. nach einer gründlichen stilistischen Untersuchung des homiletischen Euvre des Andreas von Kreta möglich wäre) bei der Textgestaltung über dem Strich den von П vertretenen Varianten der Vorzug gegeben.

${ }^{171}$ Der Verweis auf die älteren Ausgaben soll nicht nur, wie bereits ausgeführt, die Textform der ihnen als Basis dienenden Handschriften belegen, sondern dient gleichzeitig der Dokumentation des (hoffentlich erzielten) Fortschrittes in der von uns gedruckten Textform.

${ }^{172}$ Dabei werden folgende Siglen verwendet (vgl. schon oben, S. 13ff. bzw. 36ff.):

II Vind. theol. gr. 160 (9. Jahrhundert, erste Hälfte): Homilia I, Homilia III

V Vat. gr. 2079 (9. Jahrhundert, Mitte): Homilia I

E Esc. $\Phi-I I I-20$ (9. Jahrhundert, zweite Hälfte): Homilia I

P Par. gr. 766 (9. Jahrhundert, zweite Hälfte/Ende): Homilia I, Homilia III

M Mosqu. Syn. gr. 284 (9. Jahrhundert, Ende): Homilia I

G Vat. gr. 455 (10. Jahrhundert, Anfang/erste Hälfte): Homilia I

D Par. gr. 763 (10. Jahrhundert, erste Hälfte): Homilia I

K Par. gr. 1171 (10. Jahrhundert, erste Hälfte): Homilia III 
$\mathrm{Zu}$ den Lesehilfen: Iota subscriptum wurde von uns stets stillschweigend ergänzt ${ }^{173}$. Abweichungen in der Akzentuierung und in der Setzung der Spiritus werden nur dann vermerkt, wenn eine im Apparat gebotene Variante aus anderen Gründen (etwa wegen eines itazistischen Fehlers) in den Apparat aufgenommen wurde ${ }^{174}$. - Im Falle des Ny ephelkystikon wurde dieses auch dort im Text belassen, wo es von $\Pi$ gegen die „klassischen“ Regeln der Schulgrammatik (d. h. auch vor Konsonanten) gesetzt wurde ${ }^{175}$.

Die von uns gewählte Interpunktion zielt auf ein dem modernen Sprachgebrauch angepaßtes Textverständnis ab und spiegelt nicht immer die in der Transkription von $\Pi$ berücksichtigte Setzung der dort als „Lesehilfen“ dienenden Interpunktionen ${ }^{176}$ wide $^{177}$. - Große Anfangsbuchstaben werden nur bei Eigennamen ${ }^{178}$ und bei den Begriffen $\Theta \varepsilon o ́ \varsigma$ und Kúpıs, aber nicht zur Kennzeichnung eines neuen Satzbeginns verwendet.

Abweichend von den philologischen Editionsregeln werden die Lesarten im Apparat nicht nach den Zeilenzahlen des Textes ausgewiesen, sondern (nach dem Vorbild der „Monumenta Germaniae Historica“) mit Hilfe eines an dem Variantenträger im Text angebrachten Exponentenbuchstabens notiert ${ }^{179}$. Diese Vorgangsweise wurde auch deswegen gewählt, um den provisorischen Charakter unserer Probeedition zu betonen: Es sei nochmals ausdrücklich darauf

A Sinait. gr. 749 (10. Jahrhundert, Mitte/zweite Hälfte): Homilia I

C Par. gr. 1454 (10. Jahrhundert, zweite Hälfte): Homilia I

£ Aug. perg. 80 (9. Jahrhundert, zweite Hälfte): lateinische Übersetzung der Homiliae I et III

F Par. gr. 819 (16. Jahrhundert): Homilia I (Vorlage für Comb¹)

R Vat. Reg. gr. 15 (13. Jahrhundert, zweite Hälfte): Homilia I (Vorlage für Comb²)

S Par. Suppl. gr. 773 (16. Jahrhundert) Homilia III (Vorlage für Bill)

Homilia I

Comb $^{1}$ editio princeps (F. Combefis, 1644)

Comb $^{2}$ editio altera correctior (F. Combefis, 1648)

Pat I. Patusas (1710; Vorlage nicht identifiziert)

Gall Gallandis Abdruck von Comb ${ }^{1}$ (1779)

PatBla Blantes' verbesserter Abdruck von Pat (1819; Vorlage nicht identifiziert)

Mi Mignes Abdruck von Gall (1860)

Homilia III

Bill editio princeps (J. de Billy, 1577; unter dem Namen des Ioannes von Damaskos)

Mi Mignes Abdruck von Bill (1860; unter dem Namen des Andreas von Kreta)

${ }^{173}$ Die wenigen Ausnahmen betreffen jene Lesarten im Apparat, bei denen der größeren Deutlichkeit halber eine Art „diplomatischer Transkription“ verwendet wurde.

174 Auf die Fehlerhaftigkeit der im Cod. Vat. gr. 2079 weitgehend von erster Hand stammenden Spiritus und Akzente wurde nur sporadisch hingewiesen, um den Apparat nicht zu überlasten; desgleichen wurde die auf eine jüngere Hand zurückzuführende (weitgehend korrekte) Setzung von Spiritus und Akzenten im Cod. Par. gr. 766 in ihren geringen Abweichungen nicht eigens im Apparat notiert.

${ }^{175}$ Wobei die von $\Pi$ vertretene Schreibung von den anderen von uns herangezogenen Textzeugen an manchen Stellen geteilt wird, an anderen nicht, ohne daß sich darin irgendeine Systematik erkennen ließe.

${ }^{176}$ Vgl. dazu oben, S. 28.

177 Darüber hinaus bezeichnet der einfache Strich | den Kolumnenwechsel, der doppelte Strich $\|$ den Seitenwechsel in $\Pi$.

${ }^{178}$ Für den in $\Pi$ (und in den anderen Textzeugen) als nomen sacrum stets gekürzt geschriebenen Namen David wurde die Auflösung $\Delta$ auío gewählt.

${ }^{179}$ Analog wurde für die Zitate (im edierten Teil: ausschließlich aus der Bibel) kein eigener Testimonienapparat angelegt: Das Zitat wird vielmehr über dem Strich kursiv (und ohne Einsatz von Anführungszeichen) gesetzt und danach (in Klammern innerhalb des griechischen Textes) identifiziert. 
hingewiesen, daß sie nicht als definitiver Text für die von ihr repräsentierten Fragmente der Homiliae I et III in nativitatem B. M. V. des Andreas von Kreta gelten kann und will; sie ist bestenfalls eine kleine Vorarbeit zu einer noch zu unternehmenden kritischen Gesamtausgabe des homiletischen Werkes dieses bedeutenden theologischen Schriftstellers.

\section{$\mathbf{A}^{\mathbf{r}}\left(\mathbf{f} .3^{\mathbf{r}}+2^{\mathrm{v}}\right)$ (vgl. $P G$ 97, 813 A 14-C 9):}

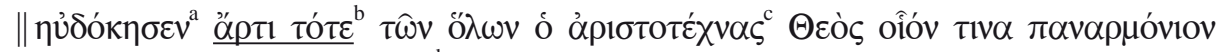

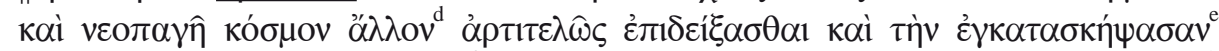

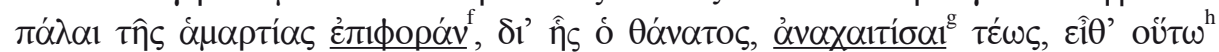

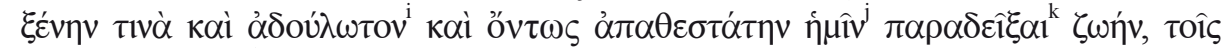

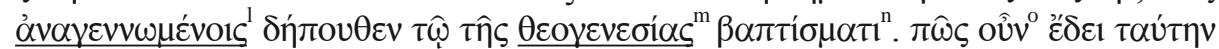

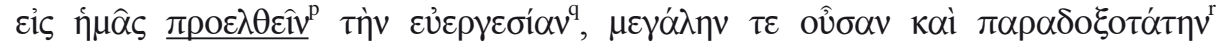

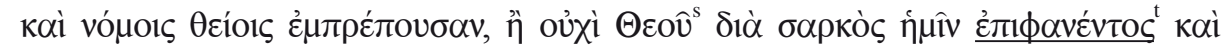

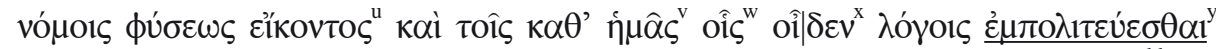

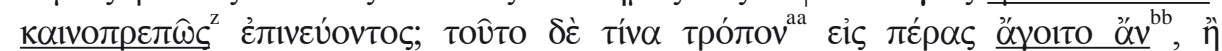

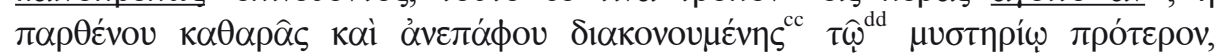

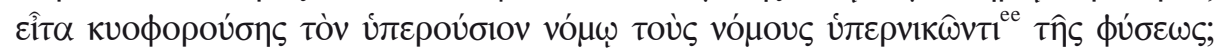

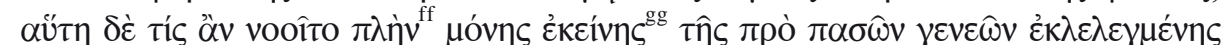

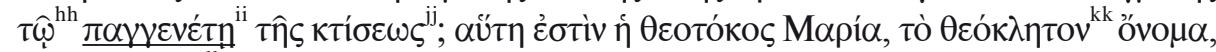

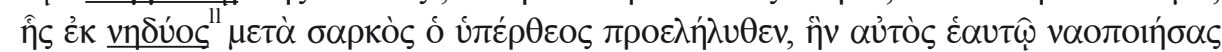

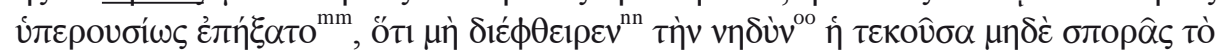

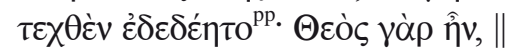

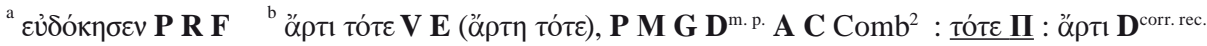

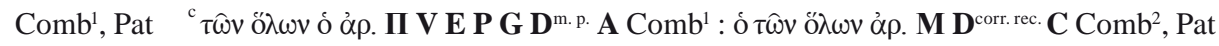

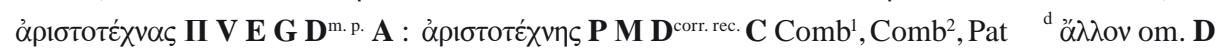

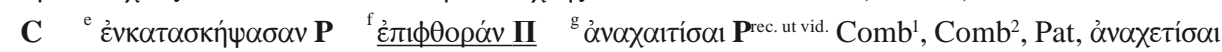

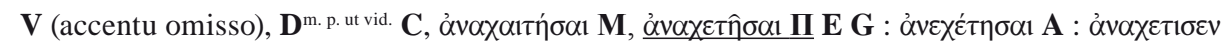

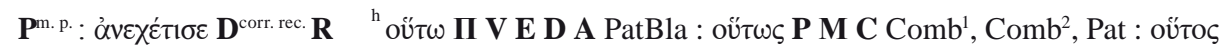

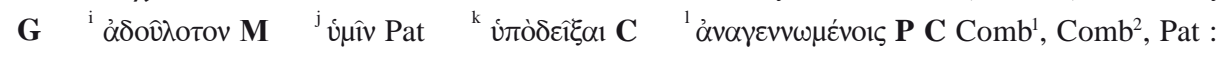

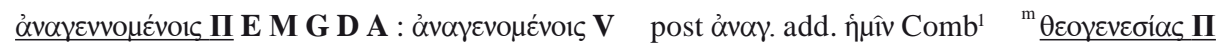

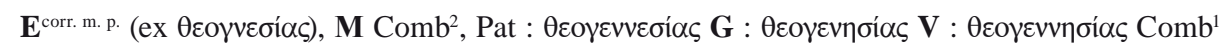

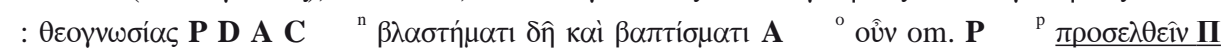

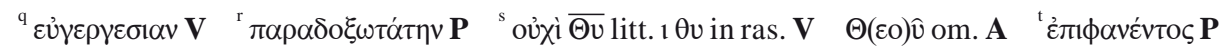

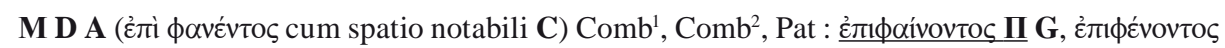

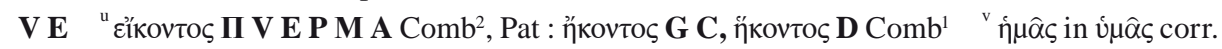

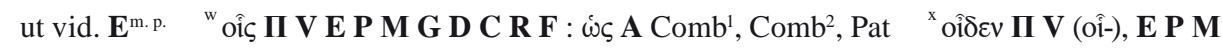

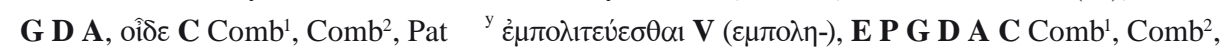

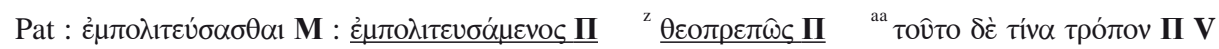

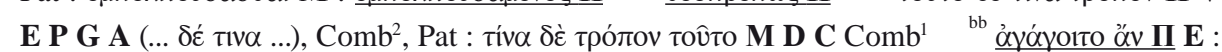

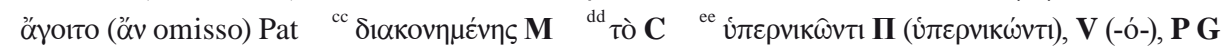

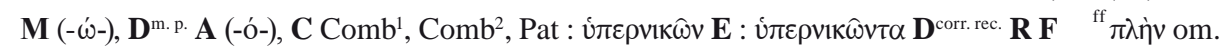

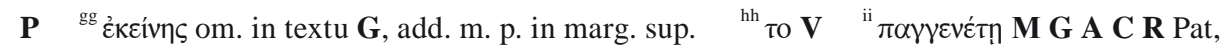

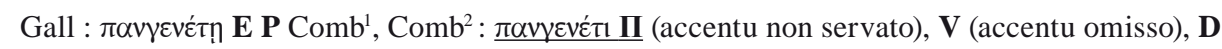

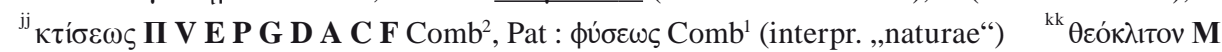

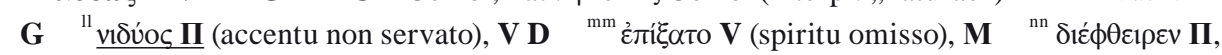

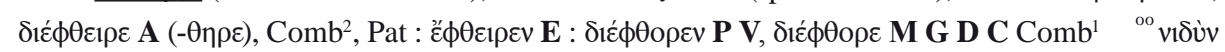
D ${ }^{\mathrm{pp}} \varepsilon \delta \delta \varepsilon \delta \varepsilon \kappa \tau o ~ P a t$
} 


\section{$\mathbf{A}^{\mathbf{v}}$ (f. $3^{\mathbf{v}}+2^{\text {r }}$ ) (vgl. PG 97, 813 C 9-816 A 5):}

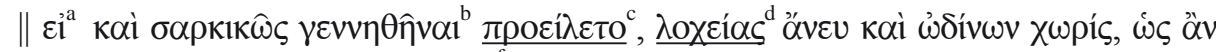

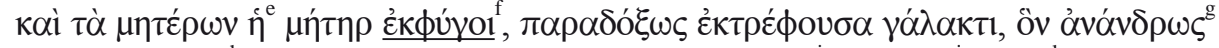

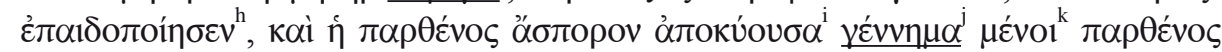

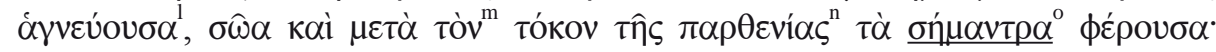

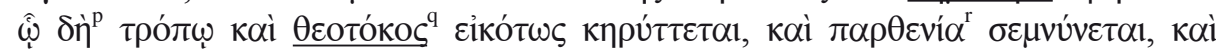

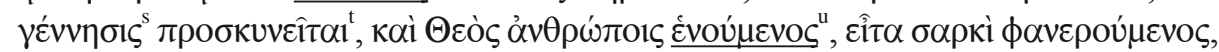

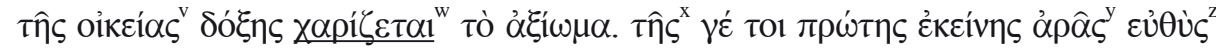

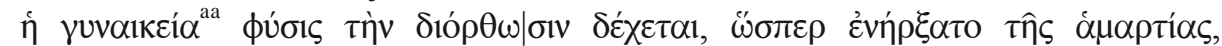

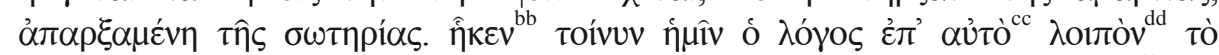

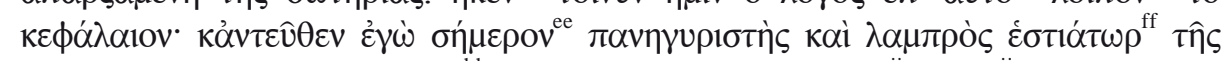

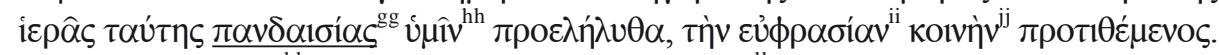

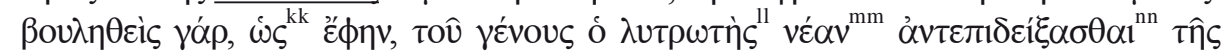

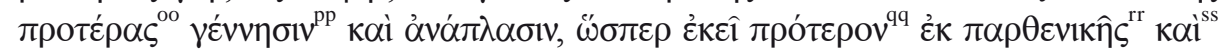

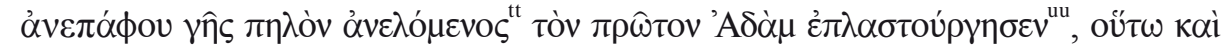

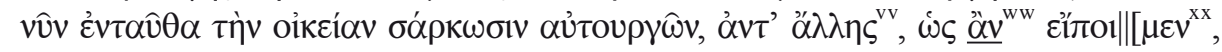

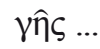

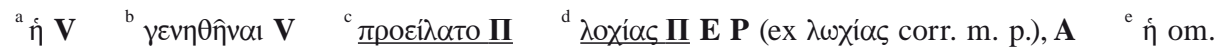

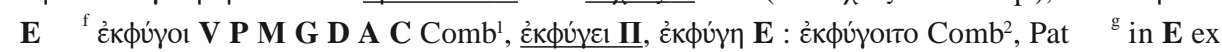

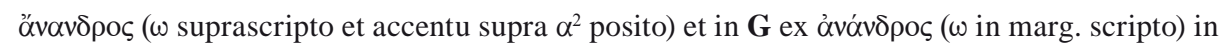

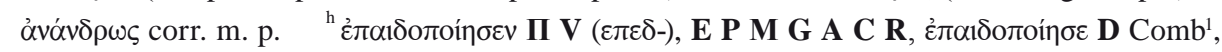

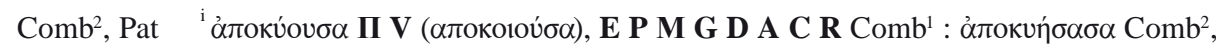

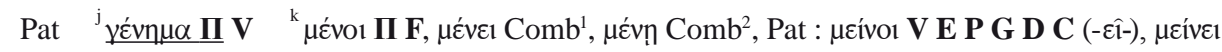

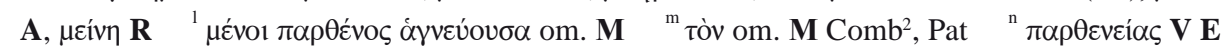

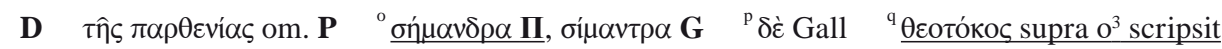

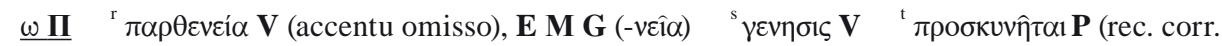

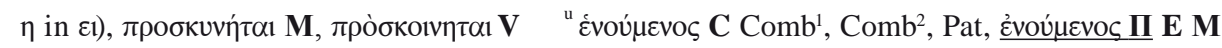
(ut vid.), G D A V (spiritu et accentu omisso), $\mathbf{P}$ (spiritu aspro manu posteriore, ut vid., addito)

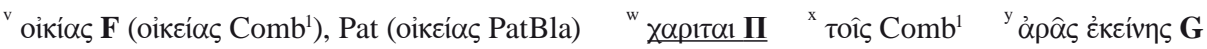

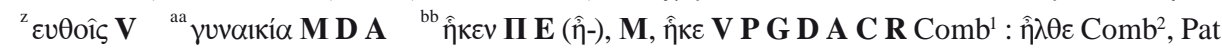

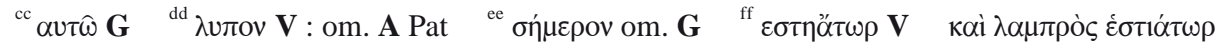

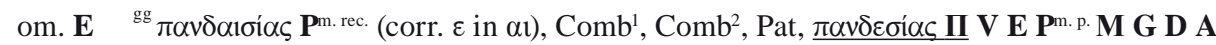

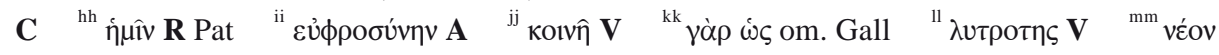

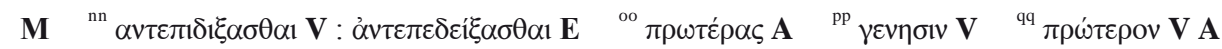

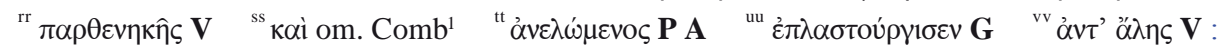

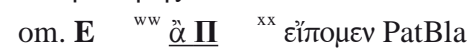

\section{$\mathbf{B}^{\mathrm{r}}$ (f. 214 $\left.{ }^{\mathrm{r}}+215^{v}\right)$ (vgl. PG 97, 817 C 12-820 A 9):}

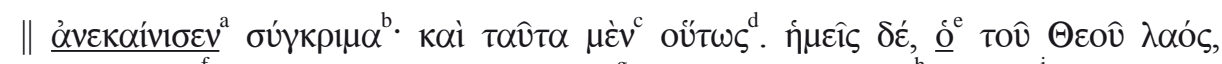

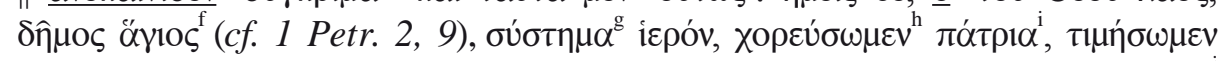

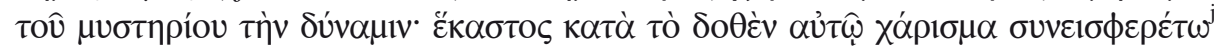

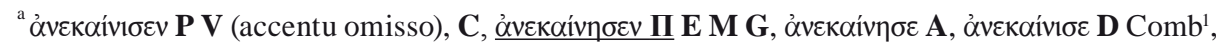

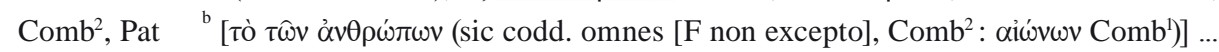

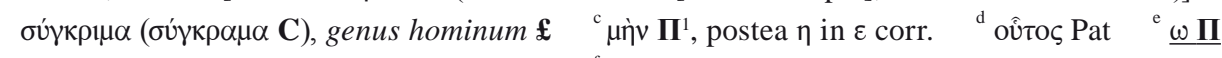

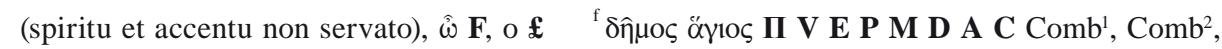

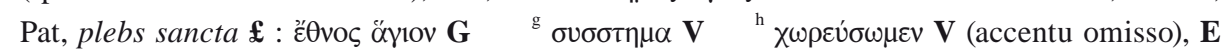

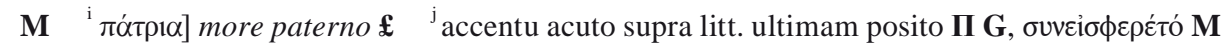




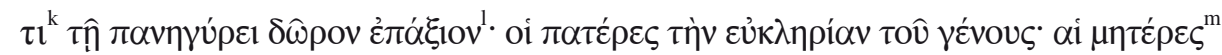

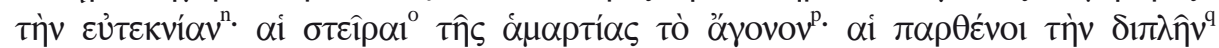

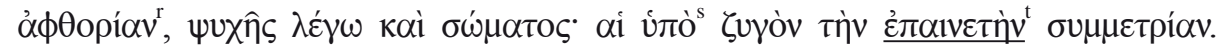

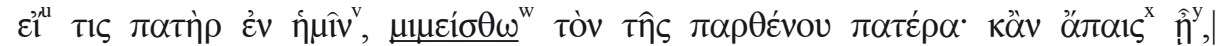

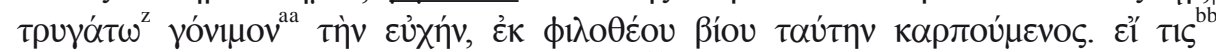

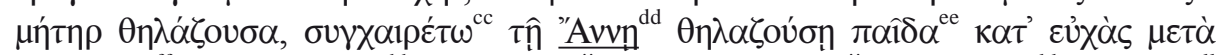

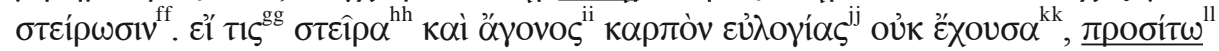

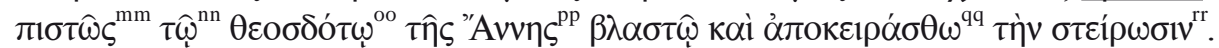

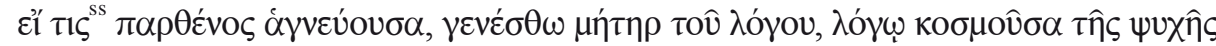

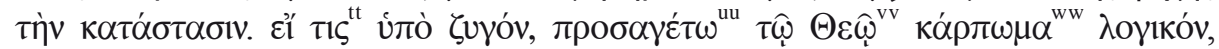

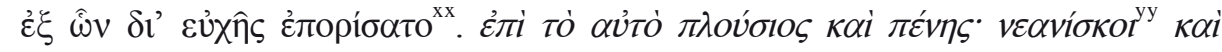
$\pi \alpha \rho \theta \dot{\varepsilon} v o l^{\cdot} \|$

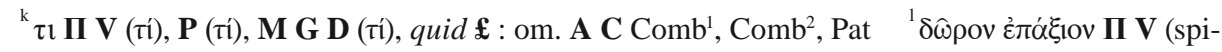

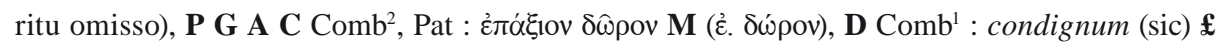

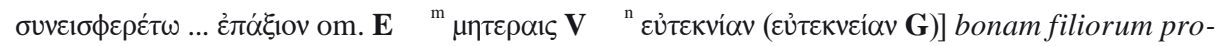

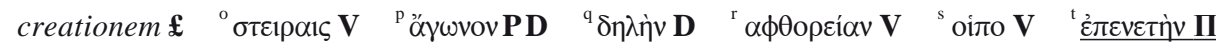

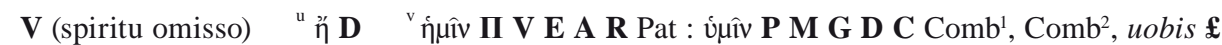

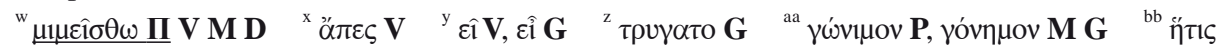

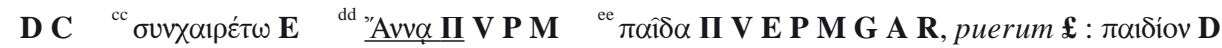

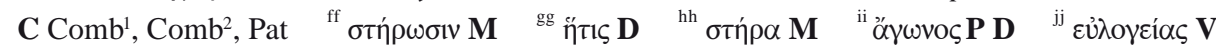

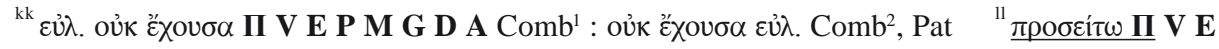

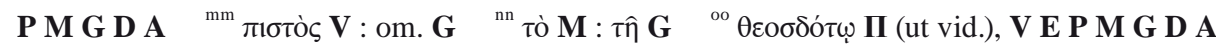

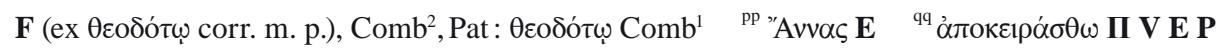

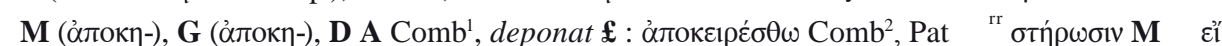

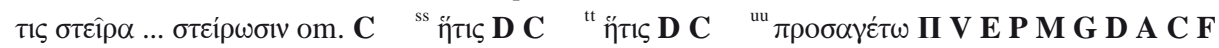

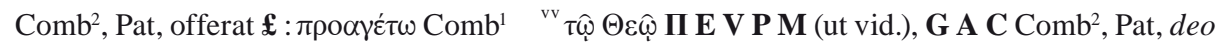

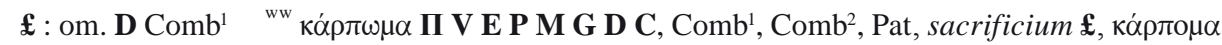

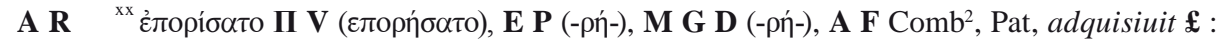

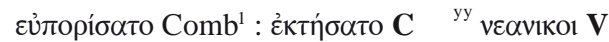

\section{$\mathbf{B}^{\mathrm{v}}$ (f. 214 $\left.{ }^{\mathrm{v}}+\mathbf{2 1 5} 5^{\mathrm{r}}\right)$ (vgl. $P G$ 97, 820 A 9-B 14):}

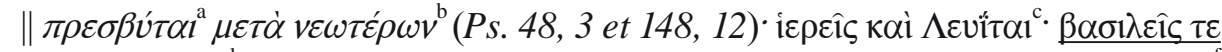

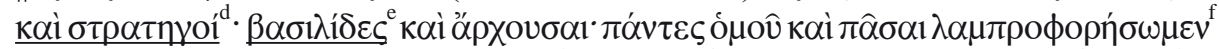

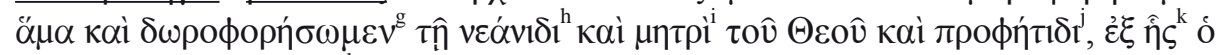

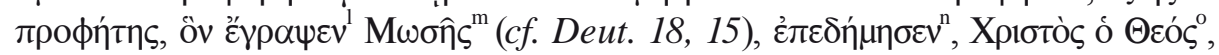

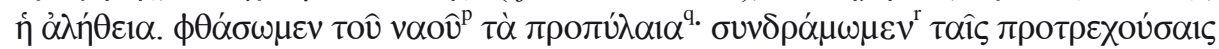

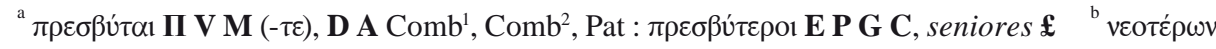

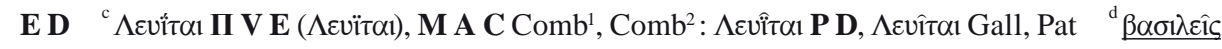

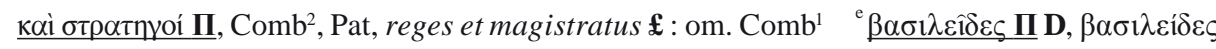

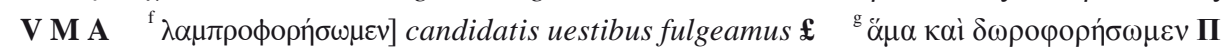

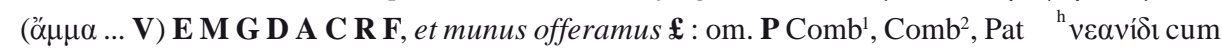

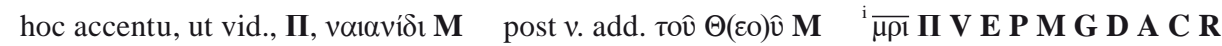

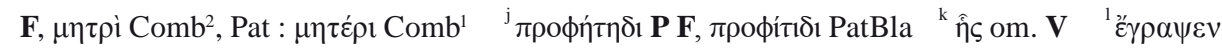
I V E P G C, है $\gamma \rho \alpha \psi \varepsilon$ M D A Comb ${ }^{1}, \mathrm{Comb}^{2}$, Pat ${ }^{\mathrm{m}} \mathrm{M} \omega \sigma \hat{\varsigma} \varsigma$ I V E M G D A C Comb ${ }^{1}$, Pat :

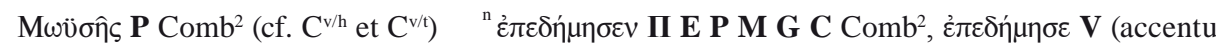

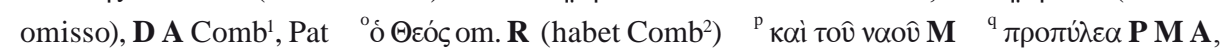

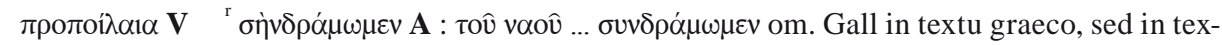
tu latino interprete Combefisio scribitur: Veniamus ad templi vestibula, una curramus cum ... 


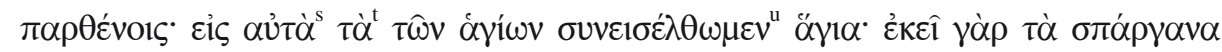

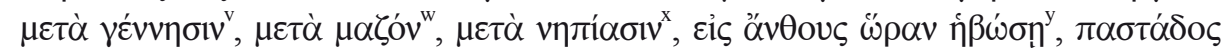

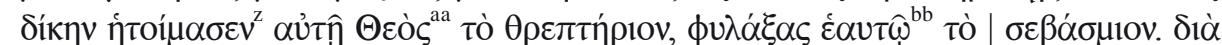

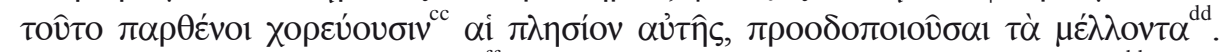

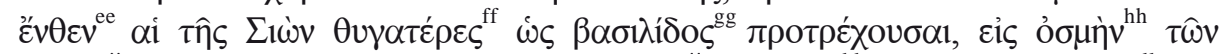

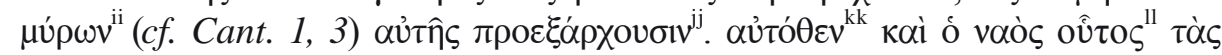

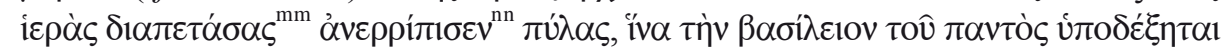

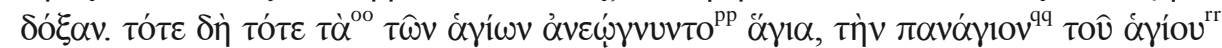

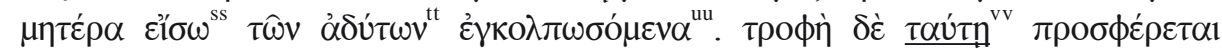

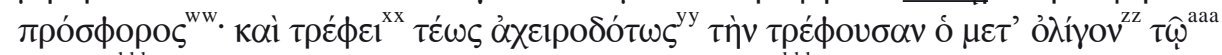

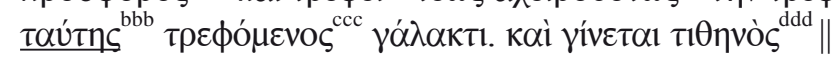

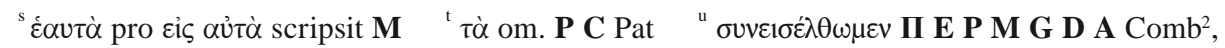

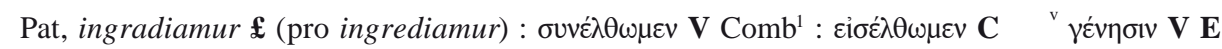

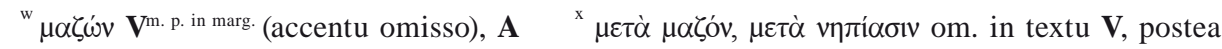

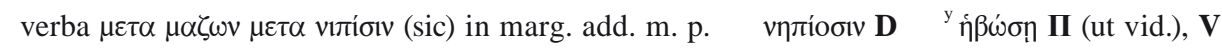

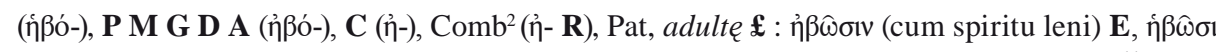

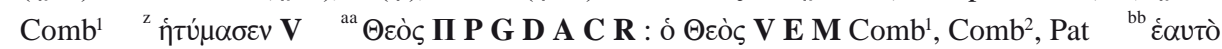

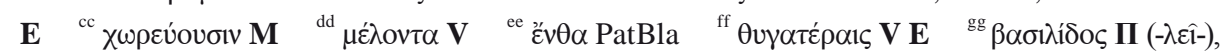

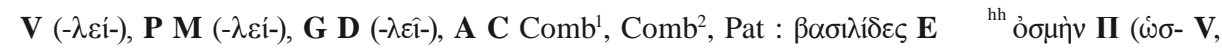

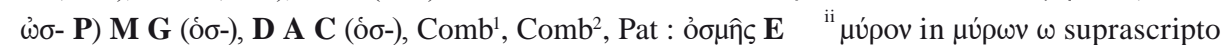

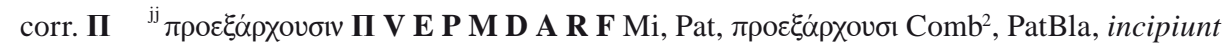

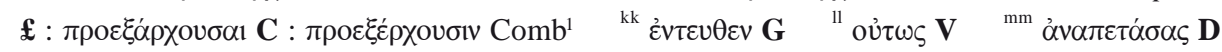

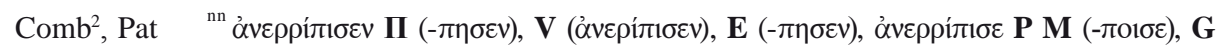

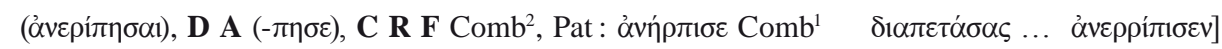

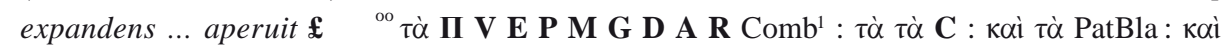

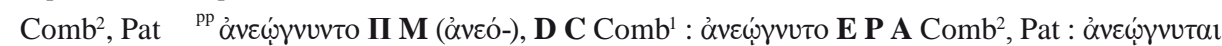

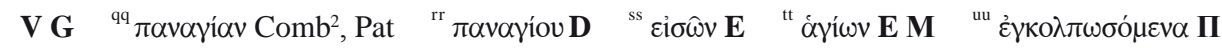

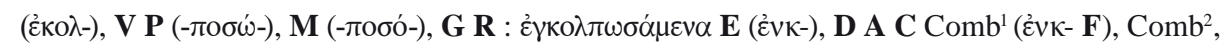

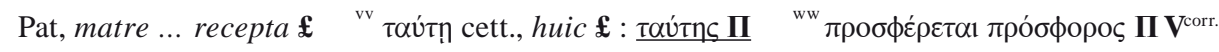

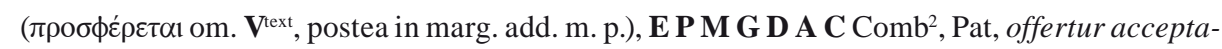

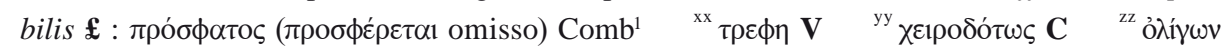

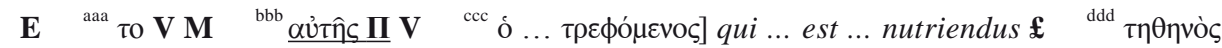
$\mathbf{V}, \tau \eta \theta i v o ̀ \varsigma ~ G$
}

\section{$\mathbf{C}^{\text {r }}\left(\mathbf{f} .4^{\text {r }}+\mathbf{1}^{\text {v }}\right)$ (vgl. PG 97, 845 A 1-B 12):}

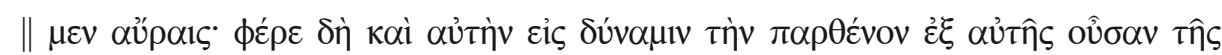

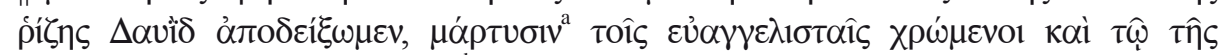

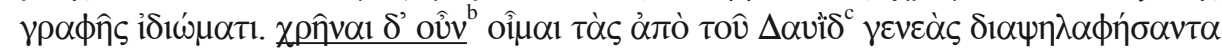

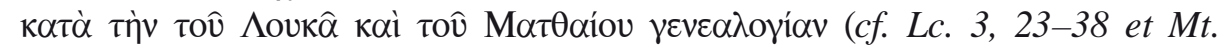

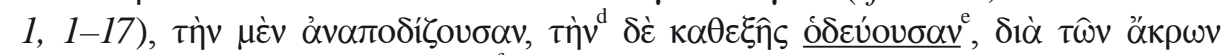

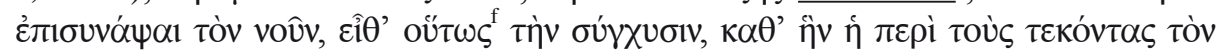

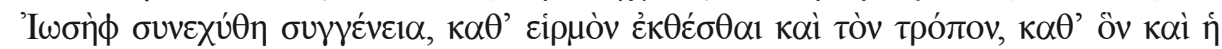

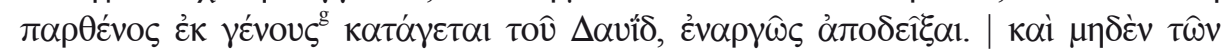

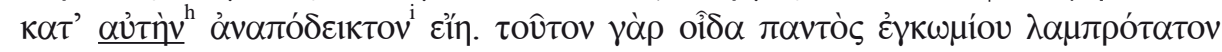

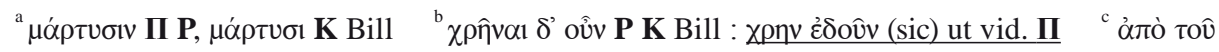

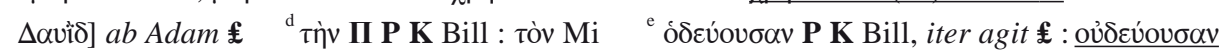

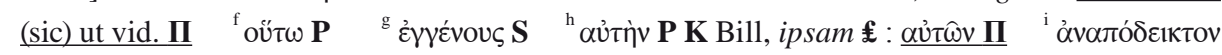

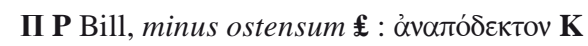




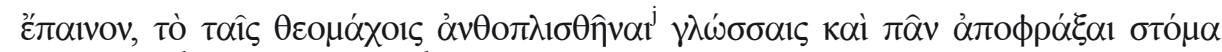

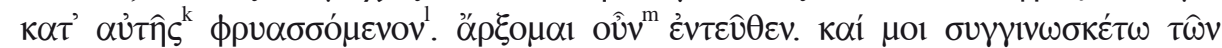

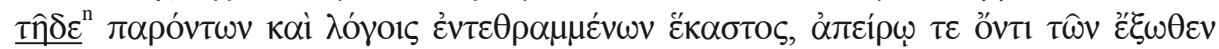

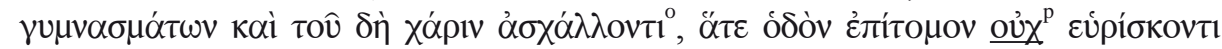

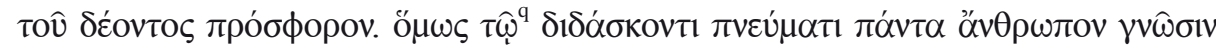

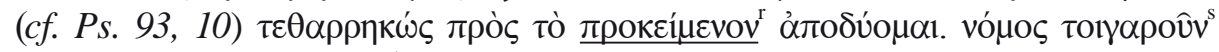

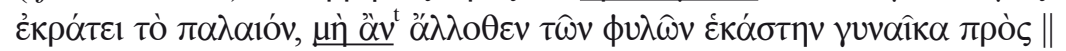

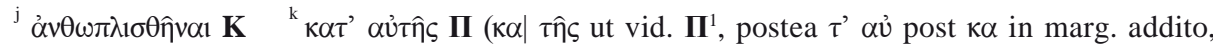

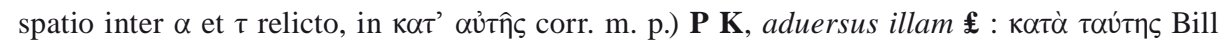

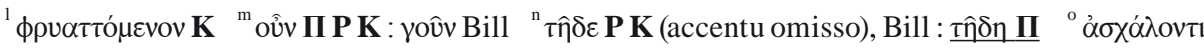

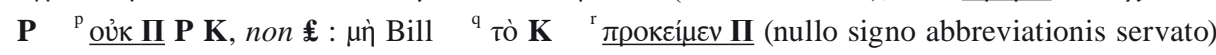

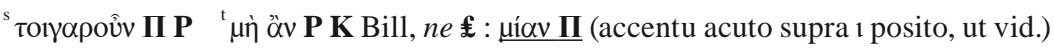

\section{$\mathbf{C}^{\mathbf{v}}\left(\mathbf{f} .4^{\mathrm{v}}+\mathbf{1}^{\mathrm{r}}\right)($ vgl. $P G$ 97, 845 B 12-D 7):}

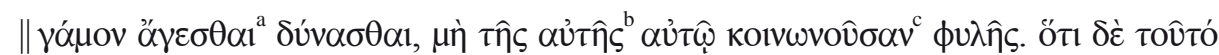

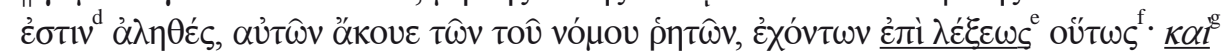

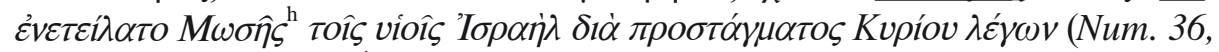

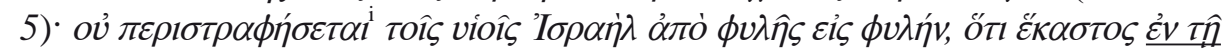

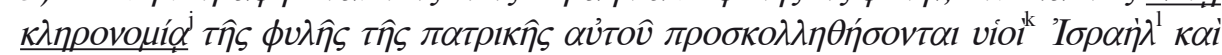

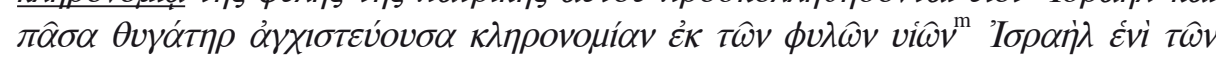

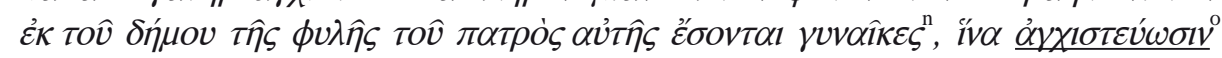

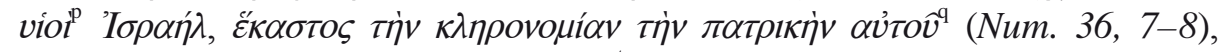

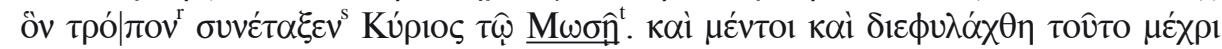

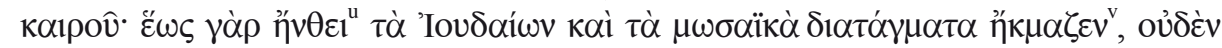

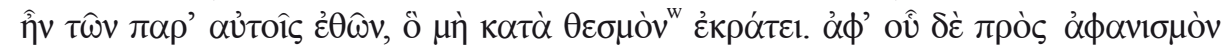

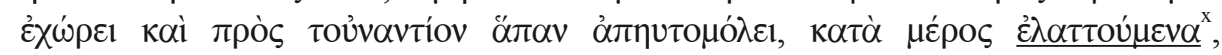

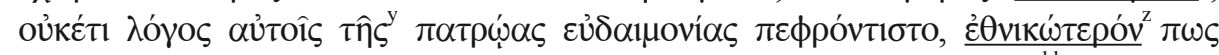

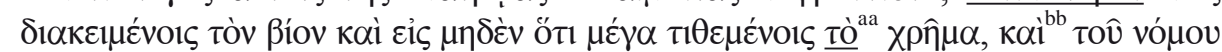

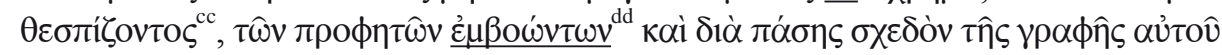

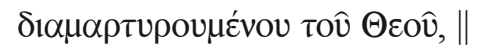

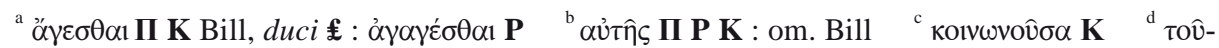

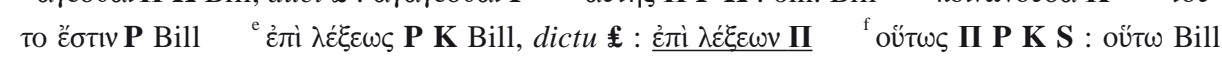
initium verborum ex Veteri Testamento allatorum litteris (ut vid.) AP (sc. ỏpı $\theta \mu o i ́)$ in interco-

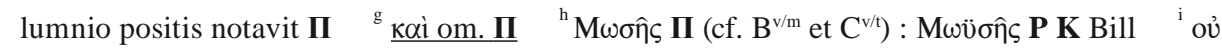

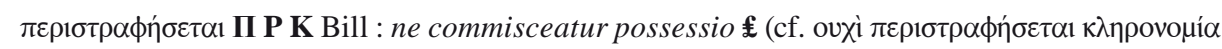

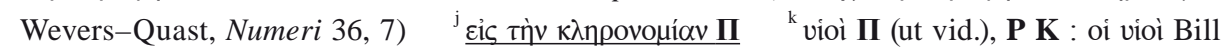

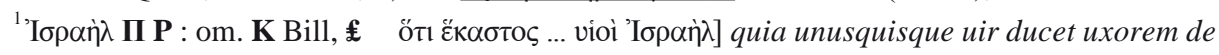

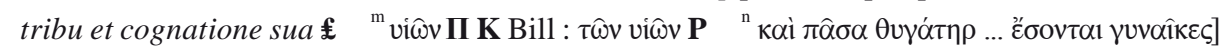

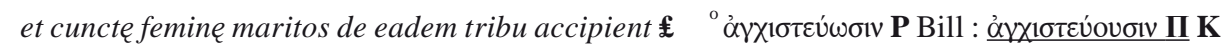

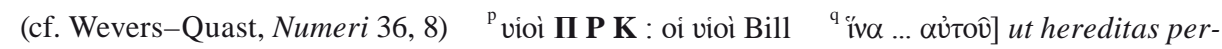
maneat in familiis nec sibi misceatur tribus, sed ita maneant, ut a domino separate sunt ${ }^{\mathrm{r}}{ }^{\mathrm{r}} \mathrm{o} \mathrm{V}$

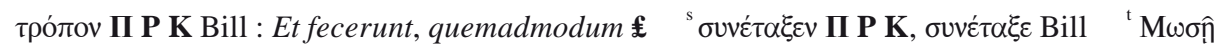
(cf. B ${ }^{v / m}$ et $C^{v / h}$ ) : M

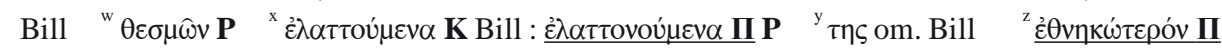

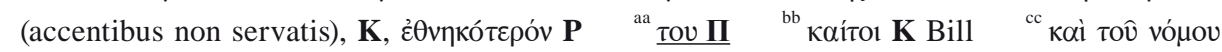

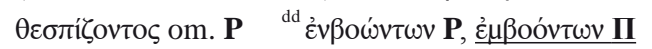




\section{AUSWERTUNG}

Aus dem textkritischen Apparat der Probeedition geht in aller Deutlichkeit hervor, daß die von unserem Fragment vertretenen Lesarten in keinem der zum Vergleich herangezogenen sonstigen Codices eine hundertprozentige Entsprechung finden ${ }^{180}$ : Manche Varianten (bzw. Fehler) in $\Pi$ werden zwar von anderen Überlieferungsträgern geteilt (aber nicht in einem Ausmaße, das einen Schluß auf eine „Familienzugehörigkeit“ zuließe) ${ }^{181}$, andere Varianten aber sind lectiones singulares bzw. errores singulares, die sich so nur in $\Pi$ finden ${ }^{182}$. Auffällig ist auf jeden Fall, daß unser Palimpsest trotz des Faktums, daß es für die Homiliae I et III in nativitatem B. M. V. der älteste erhaltene, von der Lebenszeit des Andreas von Kreta kaum mehr als 100 Jahre entfernte Textzeuge ist, keineswegs immer den besten Text zu bieten scheint ${ }^{183}$.

Die soeben getroffene Feststellung, daß die Palimpsestblätter des Cod. Vind. theol. gr. 160 als ältester Textzeuge für die Nativitätshomilen des Andreas von Kreta zu werten sind, bedarf noch einer ausführlicheren, auf paläographischen Beobachtungen aufbauenden Begründung, welche die seinerzeit im Rahmen der Katalogisierung der Codices Vindobonenses theologici graeci gebotene allgemeine Datierung ins 9. Jahrhundert ${ }^{184}$ spezifiziert ${ }^{185}$ :

Die rechtsgeneigte ogivale Majuskel des Andreas-Textes zeigt eine ausgesprochen gute und gleichmäßige (freilich nicht mit dem Epitheton „außerordentlich elegant“" zu versehende) Ausformung der einzelnen Buchstaben; die Rechtsneigung der Schriftachse ist nicht sonderlich ausgeprägt, aber durchaus einheitlich. Auffällig ist auf jeden Fall der stark betonte Wechsel in der Größe der einzelnen Buchstabenmodule, wobei sich vor allem sehr schmal gestaltete Buchstaben (wie Epsilon, Theta, Omikron und Sigma) von solchen abheben, die sich gewissermaßen in ein Quadrat einschreiben lassen. Im Gegensatz dazu ist der Kontrast zwischen dickerer und dünnerer Strichführung nicht son-

${ }^{180}$ Die in textkritischer Hinsicht gegebene Sonderstellung von $\Pi$ ließe sich auch als Argument bei der Klärung des oben ausführlich diskutierten Problems heranziehen, wie groß der Textverlust zwischen dem verstümmelten Ende der Homilia I und dem verstümmelten Beginn der Homilia III gewesen sein könnte bzw. welcher Text ursprünglich auf den nicht mehr erhaltenen Blättern (bzw. auf dem nicht mehr erhaltenen Blatt) zwischen $\mathrm{B}^{\mathrm{v}}$ und $\mathrm{C}^{\mathrm{r}}$ untergebracht war. Zur Erinnerung: Nur bei der Annahme einer gewissen Lagenanomalie - Ternio anstelle des üblichen Quaternio - wäre ein direkter Übergang von Homilia I zu Homilia III möglich; bei der Annahme eines Quaternio (und auch eines Quinio) ist die Frage, welcher Text zwischen den beiden Andreas-Homilien gestanden sein könnte, praktisch unbeantwortbar (vgl. die Zusammenfassung oben, S. 26-27). Die soeben apostrophierte Sonderstellung von $\Pi$ könnte daher die oben ausgesprochene Vermutung untermauern, daß der Blattverlust einen heute nicht mehr erhaltenen Text (scil. zu Mariä Geburt) oder eine heute nicht mehr greifbare, umfangmäßig abweichende Rezension eines der von uns namhaft gemachten Texte betroffen hat.

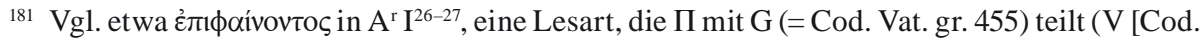

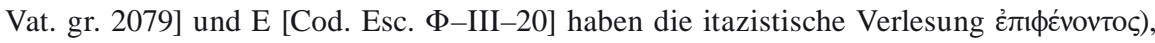
während in den anderen eingesehenen Handschriften (und in allen Ausgaben) an dieser

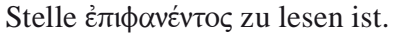

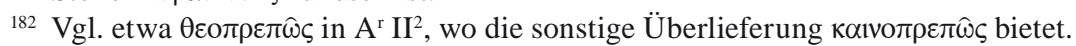

183 Auffällig ist auch, daß sich bei einem Vergleich der von П (und von E [Cod. Esc. $\Phi-I I I-20]$ ) vertretenen Textform mit jener in den von uns eingesehenen Minuskelhandschriften kein einziger Fall findet, in dem $\Pi$ und $E$ dazu beitragen, einen Translitterationsfehler Majuskel $\rightarrow$ Minuskel als solchen festzustellen.

${ }^{184}$ Vgl. oben, S. 5-6 mit Anm. 6.

${ }^{185} \mathrm{Zu}$ den folgenden Ausführungen vergleiche man das dem Beitrag beigegebene Abbildungsmaterial. 
derlich betont. All das unterscheidet die Schrift des Fragments deutlich von jenen Beispielen, die mit einiger Sicherheit dem sinaitisch-palästinensischen Raum zugewiesen werden können ${ }^{186}$. Die zwar durchaus vorhandenen, aber nicht übermäßig groß gestalteten dreieckig-zapfenförmigen Zierhäkchen (etwa bei Gamma, Delta, Theta, Tau; etwas weniger ausgeprägt bei Ypsilon und Chi) sprechen dafür, die Entstehung des ursprünglichen Homiliars mit den AndreasTexten in der ersten Hälfte des 9. Jahrhunderts anzusetzen; in dem ebenfalls in rechtsgeneigter ogivaler Majuskel geschriebenen Cod. Esc. $\Phi-$-III-20 (der oben als weiterer alter Textzeuge für die Homilia I in nativitatem B. $M$. V. vorgestellt wurde $^{187}$ ) sind diese Zierzäpfchen weitaus markanter gestaltet ${ }^{188}$, was daran denken läßt, daß er um einige Jahrzehnte jünger ist als unsere Fragmente. Hinsichtlich einer Lokalisierung der Andreas-Fragmente sind beim derzeitigen Forschungsstand keine konkreten Aussagen möglich (zumal auch die spätere Geschichte des ursprünglichen Codex, d. h. der Weg, den die Blätter vor dem 15. Jahrhundert genommen haben ${ }^{189}$, keine in dieser Richtung verwertbaren Anhaltspunkte bietet); eine Zuweisung nach Konstantinopel wäre daher eine reine, unbeweisbare Spekulation.

$\mathrm{Zu}$ den Einzelformen lassen sich folgende Beobachtungen machen: Die beiden ersten, ohne ein Absetzen geschriebenen Züge des Alpha treten in zwei verschiedenen Formen auf: Neben der häufigeren Schreibung mit einem spitzen Winkel findet sich auch eine runde Ausgestaltung links von der anschließend von links oben nach rechts unten kräftig gezogenen Hauptlinie. - Epsilon (und Sigma) sind (wie bereits gesagt) stets sehr schmal geschrieben (was ihre Unterscheidung im Palimpsest bisweilen erschwert). - Beim Theta kann der mittlere Strich manchmal sehr hoch angesetzt sein. - Die brückenartige Verbindung der beiden Hasten des My ist nicht sehr sorgfältig durchgezogen. Auch beim Xi sind die „Zacken“ unter dem oberen Balken nicht sehr sorgfältig gesetzt (was auf eine relativ hohe Geschwindigkeit beim Schreiben hindeutet). - Das Phi weist die für die rechtsgeneigte ogivale Majuskel übliche Form auf, doch tendiert der Mittel,,kreis“ eher zu eckigen, rhomboiden Formen.

Zusammenfassend läßt sich zu unserem Palimpsest sagen, daß sein ursprünglicher „Muttercodex“ (wie eingangs gesagt: mit höchster Wahrscheinlichkeit ein mit dem Beginn des Jahres am 1. September einsetzendes Homiliar) aufgrund der Verwendung der rechtsgeneigten ogivalen Majuskel, aufgrund der Zweispaltigkeit und aufgrund des Formats (ca. $340 \times 235$ mm) einen „retrospektiv“ ausgerichteten Buchtyp repräsentiert hat: Selbst der ebenfalls in einer (wohl etwas jüngeren) rechtsgeneigten ogivalen Majuskel geschriebene Cod. Esc. $\Phi-I I I-20$ ist bereits einspaltig und weist ein bescheideneres Format (202 × 142 mm) auf. Besonders auffällig ist der Unterschied zwischen $\Pi$ und dem fast gleichzeitig (d. h. um die Mitte des 9. Jahrhunderts) entstandenen Cod. Vat. gr. 2079: Dieser (mit einiger Wahrscheinlichkeit dem studitischen Milieu angehörende) Codex verkörpert von seinem Phänotyp her (Verwendung der frühen Minuskel, Einspaltigkeit, „handliches“ Format [208 × 146 mm]) eine in die Zukunft weisende Buchform.

\footnotetext{
${ }^{186}$ Vgl. etwa Cavallo, Funzione e struttura (wie in Anm. 6) 99 («tipo agiopolita» mit Bezug auf den Uspenskij-Psalter [Cod. Petropol. gr. 216 (aus dem Jahr 862/863); Abb. 5 auf S. 115]) (u. ö.); vgl. auch die Abbildungen des Cod. Sinait. gr. 210 (+ Sinait. gr. NE Meg. Perg. 12 [aus dem Jahre 861/862]) bei Harlfinger-Reinsch-SonderKamp-Prato, Specimina Sinaitica (wie in Anm. 26), Taf. 1-4 (und Frontispiz).

187 Vgl. oben., S. 13.

188 Vgl. die unserem Beitrag beigegebene Abb. 13.

${ }^{189}$ Vgl. dazu unten, S. 47-51.
} 
Bei dem Versuch, die späteren Schicksale des „Muttercodex“ (d. h. des alten Homiliars mit den Andreas-Texten) bis zu seiner Palimpsestierung ${ }^{190}$ (und danach) zu rekonstruieren, ergibt sich in erster Linie das Problem der sauberen Scheidung zwischen jenen bei der Bestimmung der Provenienz auswertbaren Notizen, die sich auf die f. 1-4 und 214-215 vor ihrer Wiederverwendung beziehen, und solchen, welche die „neuen“ Handschriften ${ }^{191}$ betreffen, die unter Heranziehung der palimpsestierten Blätter angefertigt wurden ${ }^{192}$.

Als besonders wichtig erweist sich dabei folgende Eintragung auf f. $4^{\mathrm{v}}$ : $†$

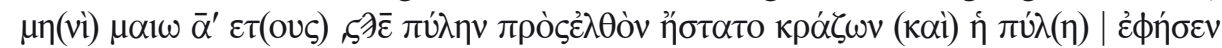

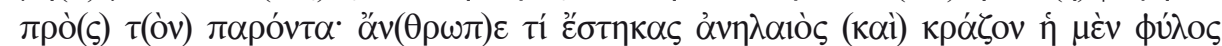

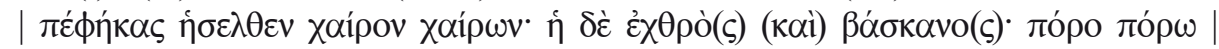

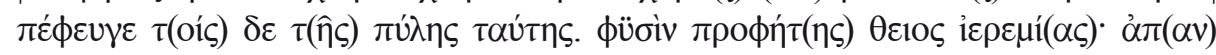

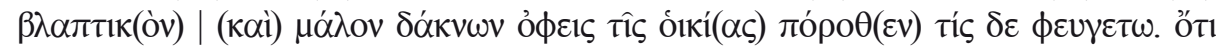

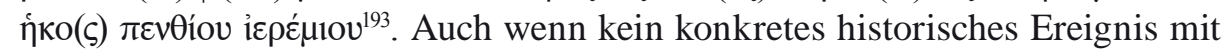
dem in dieser Notiz genannten 1. Mai 1397 in Verbindung gebracht werden kann ${ }^{194}$, so läßt ihre Position im heutigen äußeren Freirand von f. 4v (parallel zur Textrichtung der Andreas-Homilie im ursprünglichen oberen Freirand von $\mathrm{f}$. $\mathrm{C}^{\mathrm{v}}$ ) doch vermuten, daß dieses Blatt des alten Andreas-Codex (und damit auch die beiden anderen Folien, A und B) zu diesem Zeitpunkt noch nicht gefaltet und noch nicht wieder beschriftet worden war(en); die zweite Aussage wird im

190 Um keine Mißverständnisse aufkommen zu lassen, sei an dieser Stelle darauf hingewiesen, daß die Bedeutung der Wörter „Palimpsest“ bzw. „palimpsestieren“ ambivalent ist: Sie können sowohl das reine Abschaben eines ursprünglichen Textes als auch das Abschaben und die Wiederbeschriftung des Beschreibstoffes bezeichnen; im folgenden wird dieser Ausdruck vor allem in seiner (engeren) ersten Bedeutung verwendet.

191 Die Pluralform mußte hier deswegen gewählt werden, weil das Evangelienlektionar (dazu s. oben. S. 5-6 mit Anm. 2-4 bzw. gleich im folgenden), bei dessen Anfertigung (oder Ergänzung?) die drei (zu sechs umgearbeiteten) Blätter mit dem ursprünglichen AndreasText herangezogen worden waren, nicht die „Endstation“ in der Geschichte der palimpsestierten Folien bedeutet: Reste des Evangelienlektionars wurden ja ihrerseits zu Vor- und Nachsatzblättern des eigentlichen alten Buchblocks des Cod. Vind. theol. gr. 160 ,umfunktioniert“.

192 Die entsprechende Differenzierung bei HungER-KRESTEN-HANnICK, Katalog (wie in Anm. 1) bedarf einiger Korrekturen (s. gleich im folgenden).

193 Unvollständige Transkription bei Hunger-Kresten-HAnnicK, Katalog (wie in Anm. 1) 246. - Dieser nicht leicht verständliche Text könnte in (teilweise) normalisierter Transkrip-

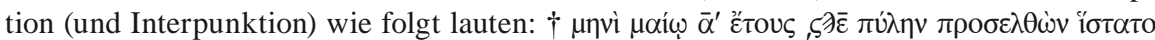

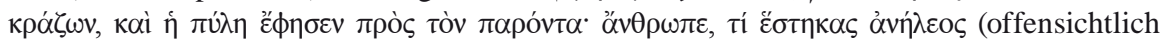

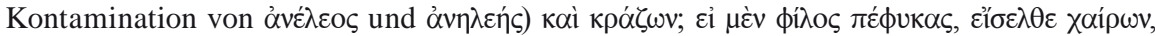

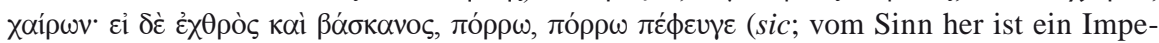
rativ 2. Person Singular zu erwarten; Aberration zu dem unmittelbar darüber stehenden

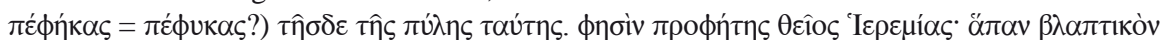

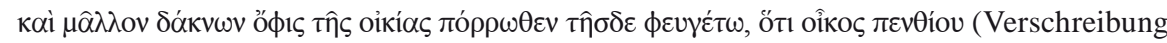

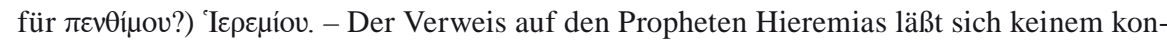

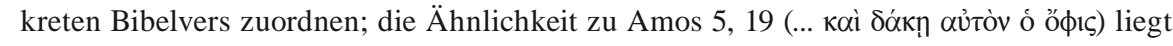
ausschließlich im Vokabular.

194 Auch dann nicht, wenn man versucht, eine Verbindung zu der auf Euboia hinweisenden Provenienz (vgl. dazu gleich im folgenden) zumindest des Hauptbuchblocks des Cod. Vind. theol. gr. 160 herzustellen. 
übrigen dadurch bestätigt, daß der sekundäre Text des Evangelienlektionars ${ }^{195}$ teilweise über dem Text dieser Notiz geschrieben ist (was im übrigen zu einer Umdatierung der Schrift des Lektionars zwingt $\left.{ }^{196}\right)^{197}$. Ob das Homiliar des 9. Jahrhunderts, dem die Homiliae I et III in nativitatem B. M. V. des Andreas von Kreta angehörten, zu diesem Zeitpunkt noch (zumindest weitgehend) unversehrt war oder ob die drei heute im Cod. Vind. theol. gr. 160 überlieferten Blätter ${ }^{198}$ bereits ihre ursprüngliche „Heimat“ verloren hatten und eine Existenz als Einzelblätter, quasi als „Makulatur“, führten, läßt sich nicht mit letzter Sicherheit entscheiden; mit hoher Wahrscheinlichkeit waren sie schon für eine Zweitverwendung vorbereitet, $d$. h. bereits palimpsestiert worden, da die letzte Zeile der Eintragung Buchstabenteile der ersten Zeile des Andreas-Textes auf f. $C^{v}$ überdeckt: Wäre die gegenständliche Notiz noch vor dem Auskratzen der Majuskel des 9. Jahrhunderts angebracht worden, wäre dieser Befund praktisch unerklärbar (da die Palimpsestierung auch diese Buchstabenteile betroffen hätte).

Wie auch immer: Wenn die in das Jahr 1397 führende Weltjahresangabe der soeben behandelten Eintragung auf f. $4^{\mathrm{v}}$ keine in die Zukunft projizierte (,prognostische“) Erfindung des (nicht sonderlich gebildeten) Schreibers dieser Notiz (oder auf eine Verschreibung bzw. auf einen „Rechenfehler“ zurückzuführen) ist, dann liefern diese Zeilen einen terminus ante quem non für die Anfertigung (oder Ergänzung) eines Evangelienlektionars unter Heranziehung der (drei) palimpsestierten Blätter des alten Homiliars - ein Befund, der mit dem paläographischen Phänotyp der Hand des Lektionars - die Schrift wurde im seinerzeitigen Katalog als „kräftige, senkrecht stehende archaisierende Minuskel“ gewertet und der ersten Hälfte des 14. Jahrhunderts zugewiesen $^{199}$ - nicht leicht in Einklang zu bringen ist. Unter der Berücksichtigung des Umstandes, daß archaisierende Minuskelschriften bisweilen nicht leicht zu datieren sind und daß die Hand des Lektionars von dem „klassischen“ Formenbestand des Archaisierens, wie er Codices etwa aus der Regierungszeit des Kaisers Andronikos II. auszeichnet ${ }^{200}$, doch sehr weit entfernt ist, scheint es legitim, sie nunmehr den ersten Dezennien des 15. Jahrhunderts zuzuweisen. Zur Gestalt und zum Umfang des Lektionars, dem die drei (nunmehr zu sechs umgearbeiteten) Blätter des alten Homiliars angehörten, lassen sich keine sicheren Aussagen machen: Es ist zwar möglich, daß sie lediglich zur Ergänzung eines älteren Lektionars dienten; ebenso denkbar (und sogar wahrscheinlicher) ist es aber, daß sie zur Anfertigung eines Lektionarheftchens herangezogen wurden, das nicht viel mehr Text bot, als heute erhalten ist (d. h. sich auf die Lesungen der Ostertage bezog): Der (zusammenhängende) Textbestand des Lektionars (f. 214 ${ }^{\mathrm{r}-\mathrm{v}} .1^{\mathrm{r}}-4^{\mathrm{v}}$. 215 $5^{\mathrm{r}-\mathrm{v}}$ ) ${ }^{201}$ bzw. das verstümmelte Ende von Joh. 3, 29 ( $\chi \alpha \rho \hat{\alpha} \chi \alpha \alpha_{1}[\rho \varepsilon \imath)$ in der letzten Zeile von f. $215^{\mathrm{v}}$ erlauben den Schluß, daß vor $\mathrm{f}$. $214^{\mathrm{r}}$ ein Blatt (wohl mit dem Text von Joh. 1, 1-17 und einer dazugehörenden

\footnotetext{
${ }^{195}$ S. dazu oben, S. 5-6.

196 Vgl. dazu auch gleich im folgenden.

197 Vgl. dazu Abb. 12.

${ }^{198}$ Dazu kommt noch mit hoher Wahrscheinlichkeit ein heute nicht mehr erhaltenes viertes Blatt, nämlich das Gegenblatt zu f. B (vgl. dazu gleich im folgenden in Anm. 202).

199 Hunger-Kresten-Hannick, Katalog (wie in Anm. 1) 245 (Hand c) und 242.

200 Vgl. dazu die jüngste Zusammenfassung bei G. De Gregorio-G. Prato, Scritture arcaizzanti in codici profani e sacri della prima età paleologa. Römische Historische Mitteilungen 45 (2003) 59-101.

${ }^{201}$ Vgl. dazu schon oben, S. 5-6 mit Anm. 2 und 4.
} 
„Gesamtüberschrift“) und nach f. $215^{\mathrm{v}}$ ein weiteres Blatt ${ }^{202}$ ausgefallen sind: Unter dieser Voraussetzung ergäbe sich ein geschlossener Quaternio, der möglicherweise schon das gesamte, auf der Grundlage der palimpsestierten Folien des alten Homiliars des 9. Jahrhunderts angefertigte „Behelfslektionar“ darstellte.

Diesen Schluß ermöglicht die Beobachtung, daß die „aktive Lebensdauer“ dieses Lektionars (ob es nun aus einem einzigen Lagenheftchen bestand oder doch umfangreicher war) relativ kurz gewesen sein muß: Sehr bald wurde es ${ }^{203}$ nämlich zu Vor- und Nachsatzblättern zum Schutz des alten, dem 11. Jahrhundert angehörenden Buchblocks des Cod. Vind. theol. gr. 160 (f. 5-210) umfunktioniert. Der genaue Zeitpunkt, zu dem dies geschehen ist, läßt sich zwar nicht eruieren, doch erlaubt das Faktum, daß sich miteinander verbundene, auf einen Handschriftentausch bezügliche und mit hoher Wahrscheinlichkeit noch der 1 . Hälfte des 15. Jahrhunderts zuzuweisende ${ }^{204}$ Provenienznotizen von der

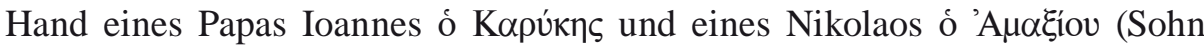
eines Pantoleon ó 'A $\mu \alpha \xi i o v)$ sowohl auf dem nunmehrigen Vorsatzblatt f. $4^{\text {v205 }}$ als auch auf dem zum Buchblock des 11. Jahrhunderts gehörenden f. 210 ${ }^{\mathrm{v} 206}$ finden, die Annahme, daß der Cod. Vind. theol. gr. 160 spätestens um das Jahr 1450 seinen heutigen Umfang (alter Buchblock + Vor- und Nachsatzblätter) erhalten haben wird.

Die Hinzufügung der palimpsestierten und mit einem Evangelienlektionar zu den Lesungen der Ostertage gefüllten Blätter A, B und C des alten Homiliars des 9. Jahrhunderts (die nunmehr zu den heute als 1-4 und 214-215 gezählten Blättern des Cod. Vind. theol. gr. 160 wurden) war freilich nicht die einzige Maßnahme, die man zur Sicherung des alten Buchblocks des Vind. theol. gr. 160 aus dem 11. Jahrhundert gesetzt hatte: Zu einem nicht näher bestimmbaren Zeitpunkt wurde die gefährdete Schlußlage f. 205-210 dieses alten Buchblocks durch das Einziehen eines schmalen Stützfalzes aus Pergament (heute f. 204/1 und f. 210/1; wiederverwertete Reste eines zweispaltigen Evangelienlektionars in senkrechter ogivaler Majuskel, wohl aus dem 10. Jahrhundert ${ }^{207}$ ) verstärkt. Da weitere Reste dieses Evangelienlektionars des 10. Jahrhunderts auch als Stützfalz (f. 210/2 und f. 212/1) um das Doppelblatt 211/212 (späterer Zusatz mit

202 Diese beiden heute nicht mehr erhaltenen Folien stammten mit hoher Wahrscheinlichkeit ebenfalls aus dem alten Homiliar des 9. Jahrhunderts; sie werden wohl durch Faltung des nunmehr verlorenen Korrespondenzblattes zu f. B $\left(214^{\mathrm{r}-\mathrm{v}}+215^{\mathrm{r}-\mathrm{v}}\right)$ des Andreas-Textes (vgl. die beiden Rekonstruktionsmöglichkeiten: oben, S. 11-12) hergestellt worden sein.

203 D. h. die sechs Folien, die heute noch erhalten sind.

204 Die Vermutung bei Hunger-Kresten-HannicK, Katalog (wie in Anm. 1) 246, diese Eintragungen könnten noch dem 14. Jahrhundert entstammen, geht von der (irrigen: vgl. das soeben Gesagte) Annahme aus, daß die Schrift des Evangelienlektionars (das auf einem Blatt, nämlich auf f. $4^{\text {v }}$, einen der hier zu diskutuierenden Vermerke trägt) der ersten Hälfte des 14. Jahrhunderts entstammt; die Richtigstellung der Datierung dieses Lektionarfragments (und seine Zuweisung in die ersten Dezennien des 15. Jahrhunderts) zwingt auch zu einer Umdatierung dieser Provenienzvermerke (die eindeutig der Gattung der „Marginalkritzeleien“ zuzuweisen sind und allein aufgrund paläographischer Kriterien kaum mit Sicherheit zeitlich einzuordnen wären). Auf der anderen Seite verweist die Alvise Sagundino († 1506) nennende (eigenhändige) Eintragung (s. gleich im folgenden) auf die zweite Hälfte des 15. Jahrhunderts, womit die termini post et ante quem für die hier diskutierten Tauschvermerke gegeben sind.

205 Eigenhändige Eintragung; Transkription bei Hunger-Kresten-HANnICK, Katalog 246.

206 Eigenhändige Eintragung; Transkription bei HungER-KRESTEN-HANNICK, Katalog 246.

207 Abbildung bei J. GrUSKOvÁ-Chr. GASTGEBER, Zur Wiederverwertung von Handschriften mit Bibeltexten am Beispiel griechischer Codices der Österreichischen Nationalbibliothek. Biblos 52 (2003), H. 1-2, 69 (Abb. 8) und 70 (Abb. 9). 
dem [schon auf dem ursprünglich frei gebliebenen Rest von f. 210v beginnenden] Kommentar des Michael Psellos zu Oratio XLIII des Gregorios von Nazianzos; platzsparende Gebrauchsschrift der zweiten Hälfte des 13. Jahrhunderts) gelegt wurden, ist daraus zu folgern, daß das Doppelblatt f. 211/212 bei dem Einziehen der Stützfalze bereits mit dem „alten“ Bestand f. 5-210 verbunden war. Offenbleiben muß die Frage, ob diese mit Hilfe der genannten Stützfalze durchgeführte „Rettungsaktion“ direkt mit der Hinzufügung der Folien 211 und 212 verbunden war (also in die zweite Hälfte des 13. Jahrhunderts zu verlegen ist) oder ob sie erst später erfolgte, spätestens im Zusammenhang mit der Beigabe der Nachsatzblätter.

Diese nach f. 212 eingefügten Nachsatzblätter setzen sich nicht nur aus den (dem alten Andreas-Codex entstammenden und dann im Evangelienlektionar des 15. Jahrhunderts wieder verwendeten) Folien 214/215 zusammen: Um dieses Doppelblatt wurden die (ebenfalls einem älteren Codex entnommenen, ebenfalls palimpsestierten und von neuem beschriebenen) Schutzblätter $\mathrm{f}$. 213/216 (untere Schrift: 12. Jahrhundert, Fragmente aus einem Menaion; obere Schrift: ebenfalls [?] 12. Jahrhundert, Fragmente aus einem Pentekostarion) ${ }^{208}$ gelegt. Damit waren die kodikologischen Maßnahmen zur Absicherung des eigentlichen Buchblocks des Cod. Vind. theol. gr. 160 endgültig abgeschlossen, und zwar ganz offensichtlich noch vor der Tauschaktion zwischen dem Papas

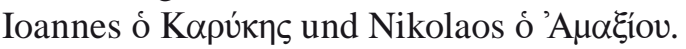

Wo diese abschließende „Rettungsaktion“ vorgenommen wurde, läßt sich nicht mit der gewünschten Eindeutigkeit bestimmen, zumal auch für die beiden soeben genannten Vorbesitzer kein genauer „Wirkungsort“ bekannt ist. Reine Spekulation bleibt es, sie mit der Insel Euboia in Verbindung zu bringen, für die sich Indizien namhaft machen lassen, die sich in dem alten Buchblock des Cod. Vind. theol. gr. 160 finden $^{209}$. Auf jeden Fall bemerkenswert ist das Faktum, daß bei den verschiedenen Etappen der Restaurierung ${ }^{210}$ des alten Buchblocks des Vindobonensis Reste von drei älteren Handschriften Verwendung fanden: das Evangelienlektionar des 10. Jahrhunderts, dem die Stützfalze f. 204/1, 210/1, 210/2 und 212/1 entstammen, das (nach dem Jahr 1397 auf der Grundlage der drei Blätter des Homiliars des 9. Jahrhunderts entstandene) Evangelienlektionar (f. 1-4 und 214/215), das die Vor- und einen Teil der Nachsatzblätter lieferte, und die Fragmente aus einem (dem 12. Jahrhundert zuzuweisenden ${ }^{211}$ ) Pentekostarion (f. 213/216), die als zusätzliche Nachsatzblätter um den Nachsatz f. 214/215 gelegt wurden. Dahingestellt sei, ob aus diesen Beobachtungen der Schluß gezogen werden darf, daß sich als Hintergrund der restauratorischen Eingriffe ein monastisches (oder zumindest kirchliches) Zentrum annehmen läßt, an dem (aus welchen Gründen auch immer als obsolet betrachtete) ältere Handschriften greifbar waren und, so sie in ihrer Gesamtheit oder in Teilen zu „Makulatur“ geworden waren, etwa nach einer entsprechenden Palimpsestierung, als Hilfsmittel bei Restaurierungen anderer Codices herangezogen werden konnten.

\footnotetext{
${ }^{208}$ Für diese Einzelheiten (vor allem zur Textidentifikation) vgl. die Angaben in der Beschreibung des Codex bei Hunger-Kresten-Hannick, Katalog (wie in Anm. 1) 242-247.

${ }^{209}$ S. gleich im folgenden.

${ }^{210}$ Grundsätzlich ist es natürlich auch denkbar, daß alle soeben beschriebenen Restaurierungsmaßnahmen zur gleichen Zeit (und am gleichen Ort) erfolgten.

${ }^{211}$ Ob sich die Vermutung einer süditalienischen Hand (,primitive, fast schülerhafte Gebrauchsschrift“), die dieses Pentekostarion (über dem reskribierten Text eines Menaion) geschrieben hat - so bei HungER-KRESTEN-HANNICK, Katalog (wie in Anm. 1) 245 -, aufrecht erhalten läßt, sei dahingestellt.
} 
Das Betreten eines in geographischer Hinsicht einigermaßen sicheren Bodens erlaubt erst eine im alten Buchblock des 11. Jahrhunderts auf f. $11^{\mathrm{v}}$ anzutreffende, der zweiten Hälfte des 15. Jahrhunderts zuzuweisende Eintragung, die ei-

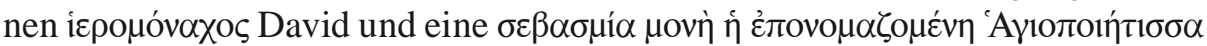

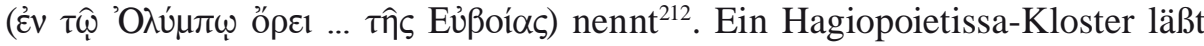
sich freilich nicht auf Euboia nachweisen ${ }^{213}$. Allerdings führt auch die nächste auswertbare Provenienznotiz, der auf f. $137^{\mathrm{r}}$ eigenhändig angebrachte Vermerk des Alvise Sagundino (ca. 1443/1443-1506) ${ }^{214}$, der geraume Zeit seines Lebens auf Euboia verbrachte ${ }^{215}$, auf diese Insel. Über Sagundino könnte der Cod. Vind. theol. gr. 160 in seinem derzeitigen Umfang nach Venedig gekommen sein $^{216}$, von wo er dann (ohne daß sich Zwischenstationen eruieren ließen) über Sebastian Tengnagel (1573-1636; Präfekt der kaiserlichen Hofbibliothek zu Wien 1608-1633) ${ }^{217}$ an die Augustissima Caesarea Bibliotheca Vindobonensis kam.

\section{ABBILDUNGSVERZEICHNIS}

Abb. 1: Cod. Vind. theol. gr. 160, f. $3^{\mathrm{r}}+2^{\mathrm{v}}\left(=\right.$ Fragment A $\mathrm{A}^{\mathrm{r}}$ (ca. 50\% der natürlichen Größe) (digitale Palimpsestphotographie) $)^{218}$. - Copyright (c) Österreichische Nationalbibliothek

Abb. 2: Cod. Vind. theol. gr. 160, f. $3^{\mathrm{v}}+2^{\mathrm{r}}\left(=\right.$ Fragment $\left.\mathrm{A}^{\mathrm{v}}\right)$ (ca. 50\% der natürlichen Größe) (digitale Palimpsestphotographie). - Copyright @ Österreichische Nationalbibliothek

${ }^{212}$ Transkription von uns hier normalisiert; vollständige diplomatische Transkription bei HuNGER-KRESTEN-HANNICK, Katalog 246.

213 Aussage auf der Grundlage von J. Koder, Negroponte. Untersuchungen zur Topographie und Siedlungsgeschichte der Insel Euboia während der Zeit der Venezianerherrschaft (Veröffentlichungen der Kommission für die Tabula Imperii Byzantini 1). Wien 1973 (vgl. besonders Fig. 11 auf S. 145); vgl. auch J. Koder-F. HiLd, Hellas und Thessalia (Tabula Imperii Byzantini 1). Wien 1976. - Das einzige Kloster, auf das die Ortsangabe év $\tau \hat{\omega}$

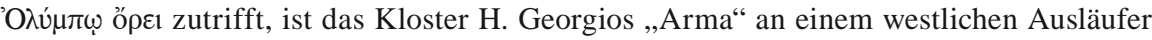
des Olympos: Koder-Hild 163; dazu kommt noch eine Kirche H. Georgios in der Nähe des Dorfes Gymnon am südöstlichen Abhang des Olympos (KoDER-HILD 168).

214 Ediert bei HungER-Kresten-HannicK, Katalog (wie in Anm. 1) 246.

${ }^{215}$ Zu Sagundino vgl. F. BABINGER, Johannes Darius (1414-1494), Sachwalter Venedigs im Morgenland, und sein griechischer Umkreis (Sitzungsber. phil.-hist. Kl. Bayer. Akad. Wiss., Jg. 1961, H. 5). München 1961, 106-107; zu seinem weitaus bekannteren und berühmteren Vater Nikolaos Sekundinos (Sagundino) vgl. die entsprechende vox in: Prosopographisches

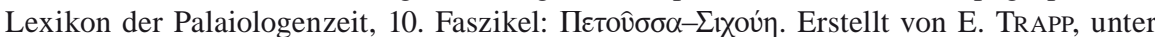
Mitarbeit von H.-V. Beyer-I. G. Leontiades-S. Kaplaneres. Wien 1990. 209-211 (Nr. 25106).

${ }^{216}$ Hinsichtlich der Schwierigkeit, die weiteren Provenienznotizen innerhalb des Codex

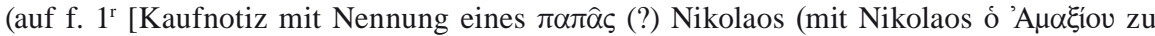

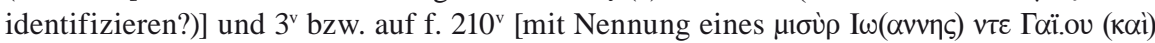
K $\alpha \sigma \tau \alpha \tau$.. (?); kopialer Beginn einer Privaturkunde? Vgl. das einleitende † ̇̀ $ү \grave{\omega}$; von dieser Hand auch eine Eintragung auf f. 59v im äußeren Freirand]) zeitlich exakt zu reihen, schließen wir uns der resignierenden Aussage bei Hunger-Kresten-HANNicK, Katalog (wie in Anm. 1) 246, an (,... chronologische Abfolge nicht einwandfrei zu bestimmen“).

${ }^{217}$ Eigenhändiges Exlibris auf f. $1^{\mathrm{r}}$ im unteren Freirand.

${ }^{218}$ Die Farbunterschiede der Abbildungen 1-12 erklären sich aus den verschiedenen Stadien der digitalen Nachbearbeitung der multispektralen Aufnahmen durch die Firma Fotoscientifica Parma und spiegeln den originalen Farbton der Blätter aus dem Cod. Vind. theol. gr. 160 nicht getreu wider. 
Abb. 3: Cod. Vind. theol. gr. 160, f. 214 ${ }^{r}+215^{v}$ (= Fragment B ${ }^{r}$ ) (ca. 50\% der natürlichen Größe) (digitale Palimpsestphotographie). - Copyright @ Österreichische Nationalbibliothek

Abb. 4: Cod. Vind. theol. gr. 160, f. $214^{v}+215^{r}$ (= Fragment Bv) (ca. 50\% der natürlichen Größe) (digitale Palimpsestphotographie). - Copyright (C) Österreichische Nationalbibliothek

Abb. 5: Cod. Vind. theol. gr. 160, f. $4^{\mathrm{r}}+1^{\mathrm{v}}$ (= Fragment $\mathrm{C}^{\mathrm{r}}$ ) (ca. 50\% der natürlichen Größe) (digitale Palimpsestphotographie). - Copyright @ Österreichische Nationalbibliothek

Abb. 6: Cod. Vind. theol. gr. 160, f. $4^{\mathrm{v}}+1^{\mathrm{r}}\left(=\right.$ Fragment $\mathrm{C}^{\mathrm{v}}$ ) (ca. 50\% der natürlichen Größe) (digitale Palimpsestphotographie). - Copyright @ Österreichische Nationalbibliothek

Abb. 7: Cod. Vind. theol. gr. 160, f. $3^{r}+2^{v}\left(A^{r}\right)$ : obere Schrift (ca. 50\% der natürlichen Größe) (digitale Photographie). - Copyright (C) Österreichische Nationalbibliothek

Abb. 8: Cod. Vind. theol. gr. 160, f. $3^{\mathrm{v}}+2^{\mathrm{r}}\left(\mathrm{A}^{\mathrm{v}}\right)$ : obere Schrift (ca. 50\% der natürlichen Größe) (digitale Photographie). - Copyright $($ O Österreichische Nationalbibliothek

Abb. 9: Cod. Vind. theol. gr. 160, f. $214^{r}+215^{v}\left(B^{r}\right)$ : obere Schrift (ca. 50\% der natürlichen Größe) (digitale Photographie). - Copyright @ Österreichische Nationalbibliothek

Abb. 10: Cod. Vind. theol. gr. 160, f. $214^{v}+215^{r}\left(B^{v}\right)$ : obere Schrift (ca. 50\% der natürlichen Größe) (digitale Photographie). - Copyright @ Österreichische Nationalbibliothek

Abb. 11: Cod. Vind. theol. gr. 160, f. $4^{\mathrm{r}}+1^{\mathrm{v}}\left(\mathrm{C}^{\mathrm{r}}\right)$ : obere Schrift (ca. 50\% der natürlichen Größe) (digitale Photographie). - Copyright ( $)$ Österreichische Nationalbibliothek

Abb. 12: Cod. Vind. theol. gr. 160, f. $4^{\mathrm{v}}+1^{\mathrm{r}}\left(\mathrm{C}^{\mathrm{v}}\right)$ : obere Schrift (ca. 50\% der natürlichen Größe) (digitale Photographie). - Copyright (C) Österreichische Nationalbibliothek

Abb. 13: Cod. Esc. $\Phi-I I I-20$ (gr. 239), f. 9r (ca. 90\% der natürlichen Größe). - Copyright $\mathbb{C}$ Patrimonio Nacional

Abb. 14: Cod. Vat. gr. 2079, f. 109v (82\% der natürlichen Größe). - Copyright @ Biblioteca Apostolica Vaticana

Abb. 15: Cod. Par. gr. 766, f. 145r (68\% der natürlichen Größe). - Copyright @ B Bibliothèque nationale de France

Sondertafelteil I: Interpunktion

Sondertafelteil II: Nomina sacra

\section{HANDSCHRIFTENVERZEICHNIS}

\section{Alexandrien}

Alexandr. Patr. 385 (173): 22 (mit Anm. 106)

\section{Athen}

Athen. Ethn. Bibl. 217: 17 (mit Anm. 83)

Athen. Ethn. Bibl. 224: 21 (mit Anm. 103)

Athen. Ethn. Bibl. 2734 (ex Kastoria 14): 1920 (mit Anm. 96)

\section{Athos}

Athous Dion. 168 (Lambros 3702): 22 (mit Anm. 108)

Athous Iber. 427 (Lambros 4547): 22 (mit Anm. 105)

Athous Meg. Laur. $\Delta$ 78: 17 (mit Anm. 81)

Athous Meg. Laur. E 160: 17 (mit Anm. 84)

Athous Prot. 57: 19 (mit Anm. 95)

Athous Simonopetr. 44 (Lambros 1312): 22 23 (mit Anm. 109)

Athous Vatop. 454: 19 (mit Anm. 92)

\section{Berlin}

Berol. gr. 43 (Phillipps 1447, Meerm. 90): 21 (mit Anm. 101)

\section{Chalke, s. Istanbu}

\section{Eskorial}

Esc. Ф-III-20 (gr. 239) (E): 13-14 (mit Anm. 44-47), 25, Anm. 125, 38, Anm. 172, 40-43, 45, Anm. 181 und 183, 46, 66 (Abb. 13: f. 9r)

\section{Florenz}

Laur. IV 4: 18 (mit Anm. 89)

\section{Istanbul}

Chalc. Panag. Kamariotisses 1: 20 (mit Anm. 97)

Chalc. Panag. Kamariotisses 47: 21 (mit Anm. 102)

Chalc. Theol. Schol. 33 (29): 22 (mit Anm. 107)

Cpl. Metochion Pan. Taphou 244: 21-22 (mit Anm. 104)

\section{Karlsruhe}

Aug. perg. 80 (£): 23-24 (mit Anm. 112-118), 38-39, Anm. 172, 41-44 


\section{Kastoria, s. Athen}

\section{Lesbos}

Lesb. Leim. 13: 17-18 (mit Anm. 86)

\section{Mailand}

Ambros. C 123 sup.: 16 (mit Anm. 77)

Ambros. C 124 sup.: 17 (mit Anm. 80)

\section{Messina}

Messin. Univ. S. Salv. 4: 16-17 (mit Anm. 78)

\section{Moskau}

Mosqu. Syn. gr. 284 (Vlad. 215) (M): 14 (mit Anm. 51-53), 38, Anm. 172, 40-43

\section{Oxford}

Oxon. Bodl. Auct. E. 2. 6 (Misc. 34): 17 (mit Anm. 79 und 82)

Oxon. Bodl. Seld. 46 (= Arch. Seld. B 53): 18 (mit Anm. 87)

\section{Paris}

Par. gr. 763 (D): 15 (mit Anm. 59-63), 38, Anm. 172, 40-43

Par. gr. 766 (P): 14 (mit Anm. 48-50), 16, 24-25 (mit Anm. 119, 121 und 125), 38, Anm. 172, 39, Anm. 174, 40-44, 68 (Abb. 15: f. 145r)

Par. gr. 819 (F): 20 (mit Anm. 98 und 99), 36 (mit Anm. 158), 38-39, Anm. 172, 40-43

Par. gr. 923: 6, Anm. 6

Par. gr. 1171 (K): 15-16 (mit Anm. 64-68), 36-37, Anm. 162, 38, Anm. 172, 43-44

Par. gr. 1454 (C): 16 (mit Anm. 72-74), 38-39, Anm. 172, 40-43

Par. gr. 2389: 9-10, Anm. 24 und 26
Par. Coisl. 274: 19-20, Anm. 95, 20-21 (mit Anm. 100)

Par. Suppl. gr. 773 (S): 37 (mit Anm. 168), 38-39, Anm. 172, 43-44

\section{Patmos}

Patm. 380: 17 (mit Anm. 82)

\section{Sinai}

Sinait. gr. 749 (526) (A): 14-15, Anm. 53, 16 (mit Anm. 69-71), 38-39, Anm. 172, 40-43

Sinait. gr. 210 (+ Sinait. gr. NE Meg. Perg. 12): 9-10, Anm. 26, 46, Anm. 186

\section{Smyrna}

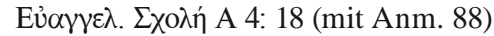

\section{St. Petersburg}

Petropol. gr. 216: 46, Anm. 186

\section{Vatikan}

Vat. gr. 455 (G): 15 (mit Anm. 54-58), 26, Anm. 138, 38, Anm. 172, 40-43, 45, Anm. 181

Vat. gr. 654: 18 (mit Anm. 90)

Vat. gr. 1882: 18-19 (mit Anm. 91)

Vat. gr. 2079 (V): 13 (mit Anm. 40-43), 26 (mit Anm. 133), 38, Anm. 172, 39, Anm. 174, 40-43, 45, Anm. 181, 46, 67 (Abb. 14: f. 109v)

Vat. Pal. gr. 205: 14, Anm. 47

Vat. Reg. gr. 15 (R): 19 (mit Anm. 93 und 94), 36 (mit Anm. 161 und 162), 38-39, Anm. 172, 40-43

Vat. Urb. gr. 35: 15, Anm. 55

\section{Wien}

Vind. theol. gr. 160 (II): passim 


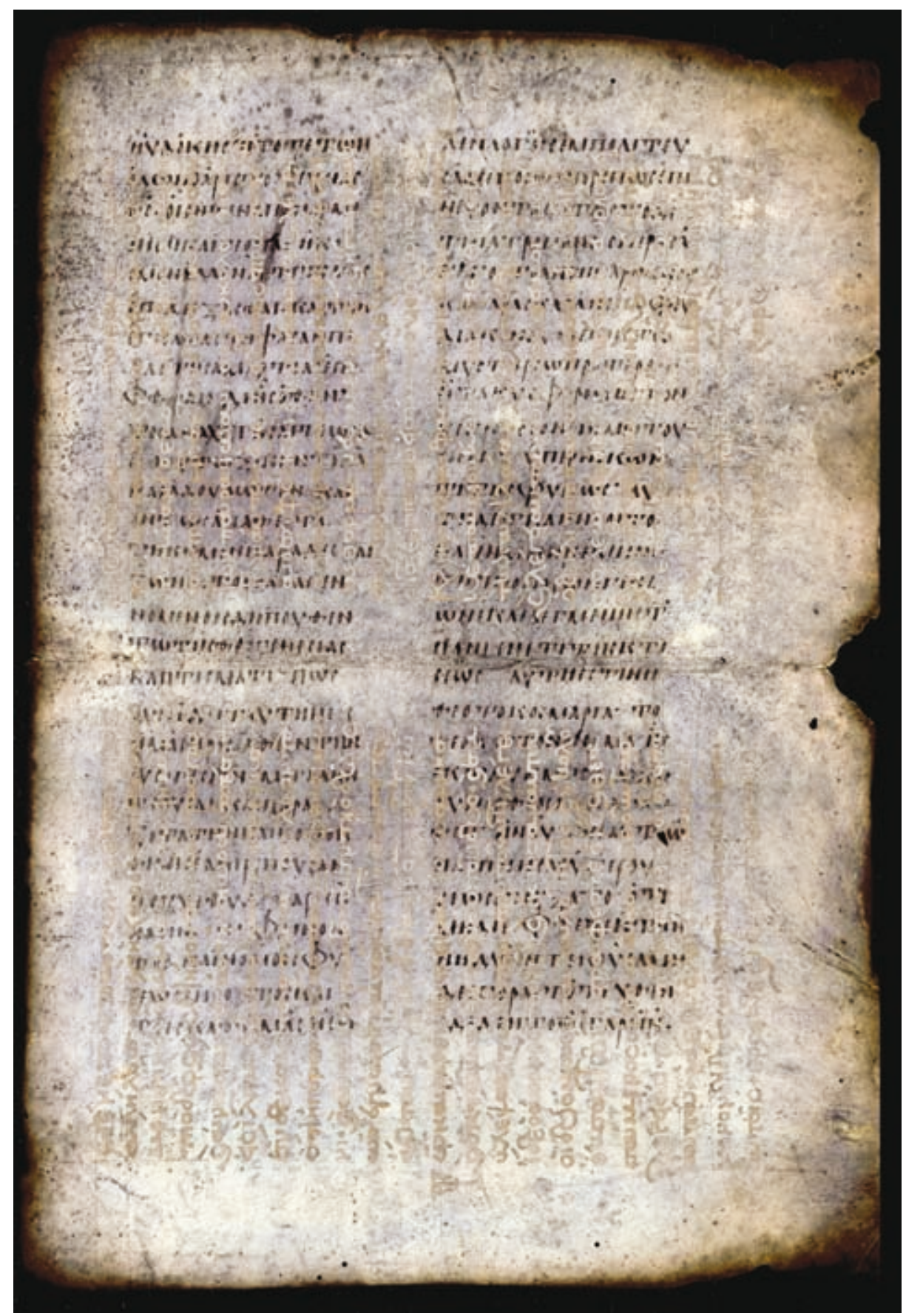

Abb. 1: Cod. Vind. theol. gr. 160, f. $3^{\mathrm{r}}+2^{\mathrm{v}}$ (= Fragment $\mathrm{A}^{\mathrm{r}}$ )

(ca. 50\% der natürlichen Größe) (digitale Palimpsestphotographie)

Copyright (c) Österreichische Nationalbibliothek 


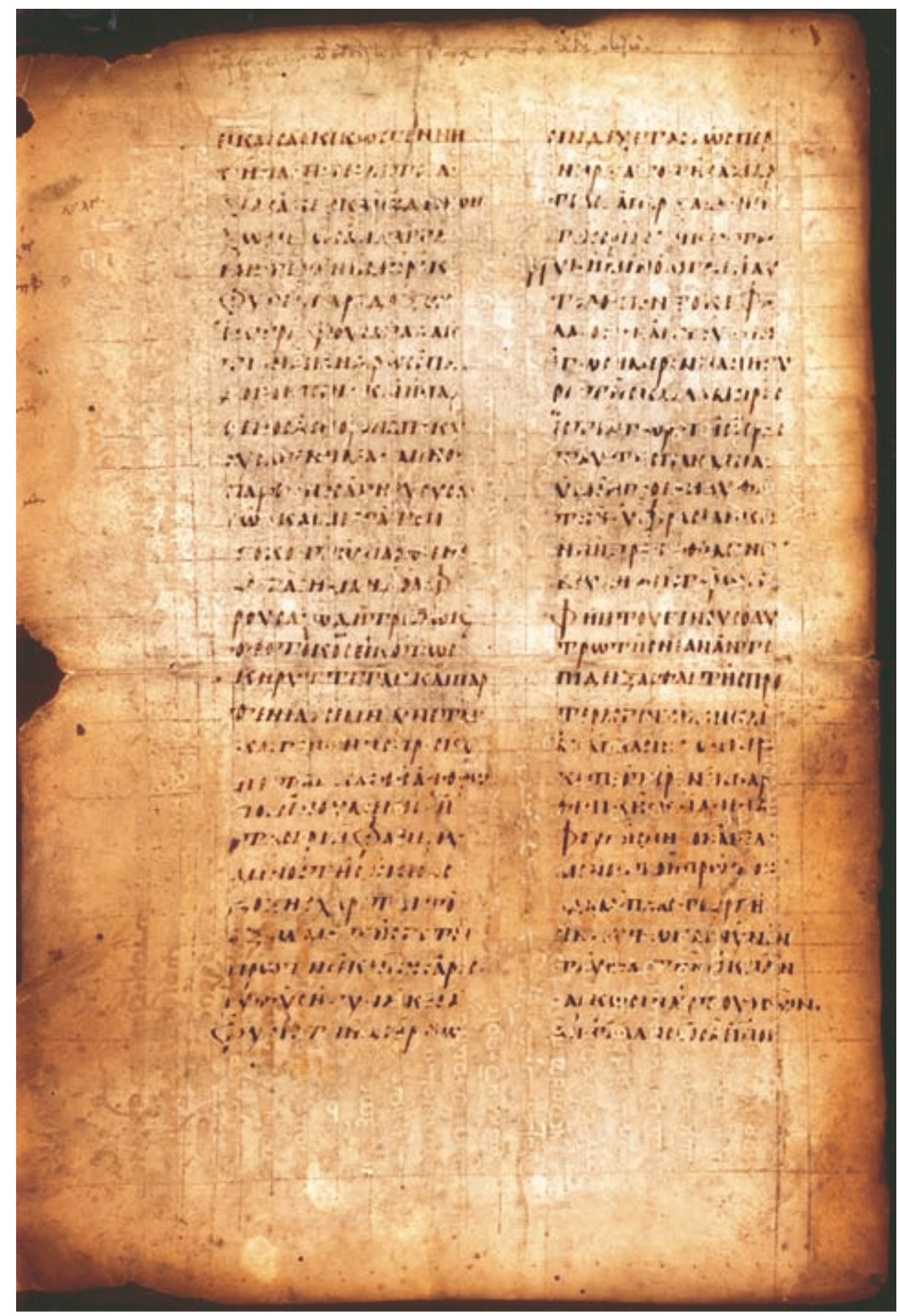

Abb. 2: Cod. Vind. theol. gr. 160, f. $3^{\mathrm{v}}+2^{\mathrm{r}}\left(=\right.$ Fragment $\mathrm{A}^{\mathrm{v}}$ )

(ca. 50\% der natürlichen Größe) (digitale Palimpsestphotographie) Copyright (C) Österreichische Nationalbibliothek 


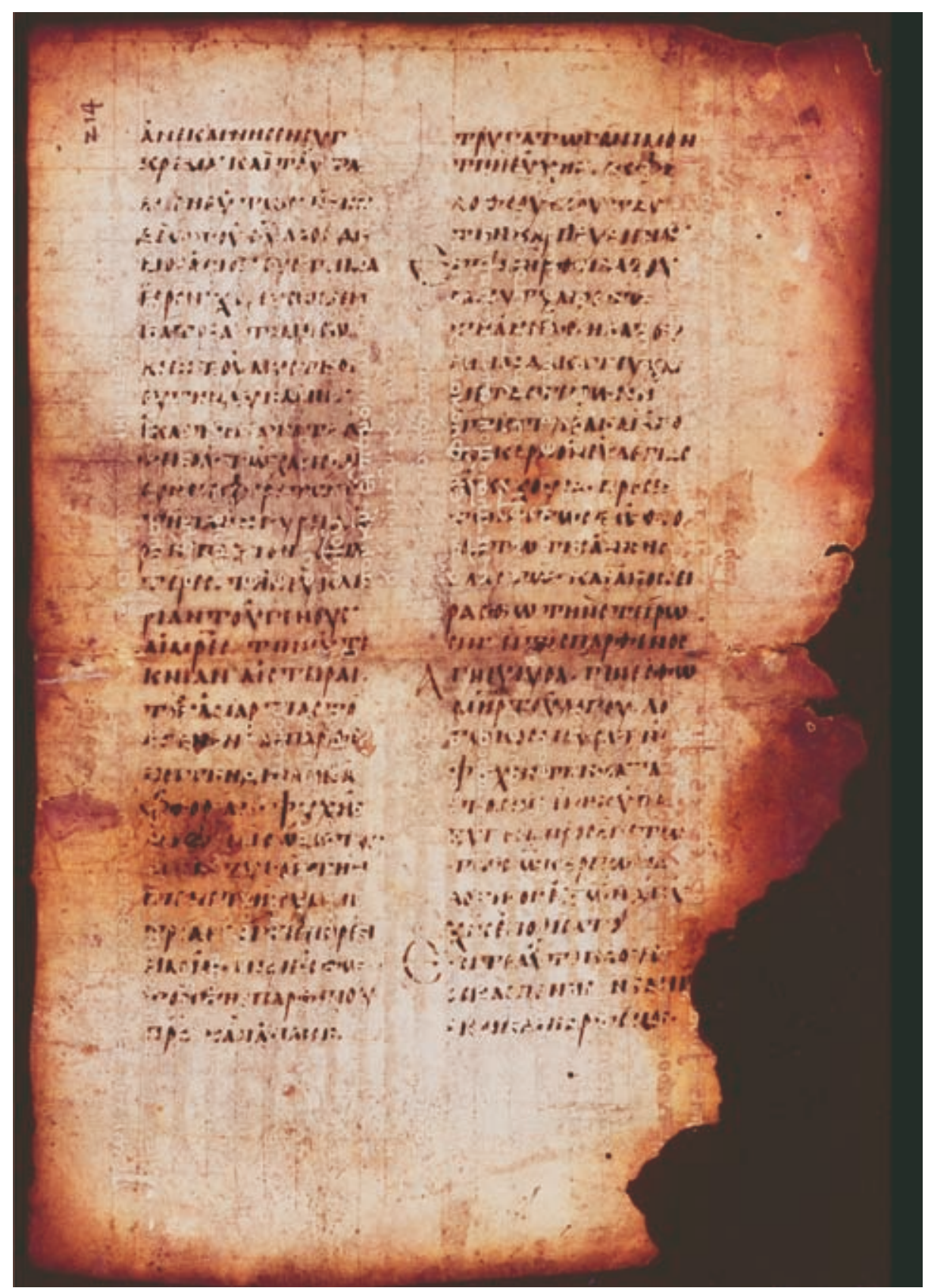

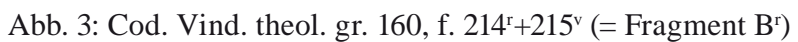

(ca. 50\% der natürlichen Größe) (digitale Palimpsestphotographie) Copyright (C) Österreichische Nationalbibliothek 


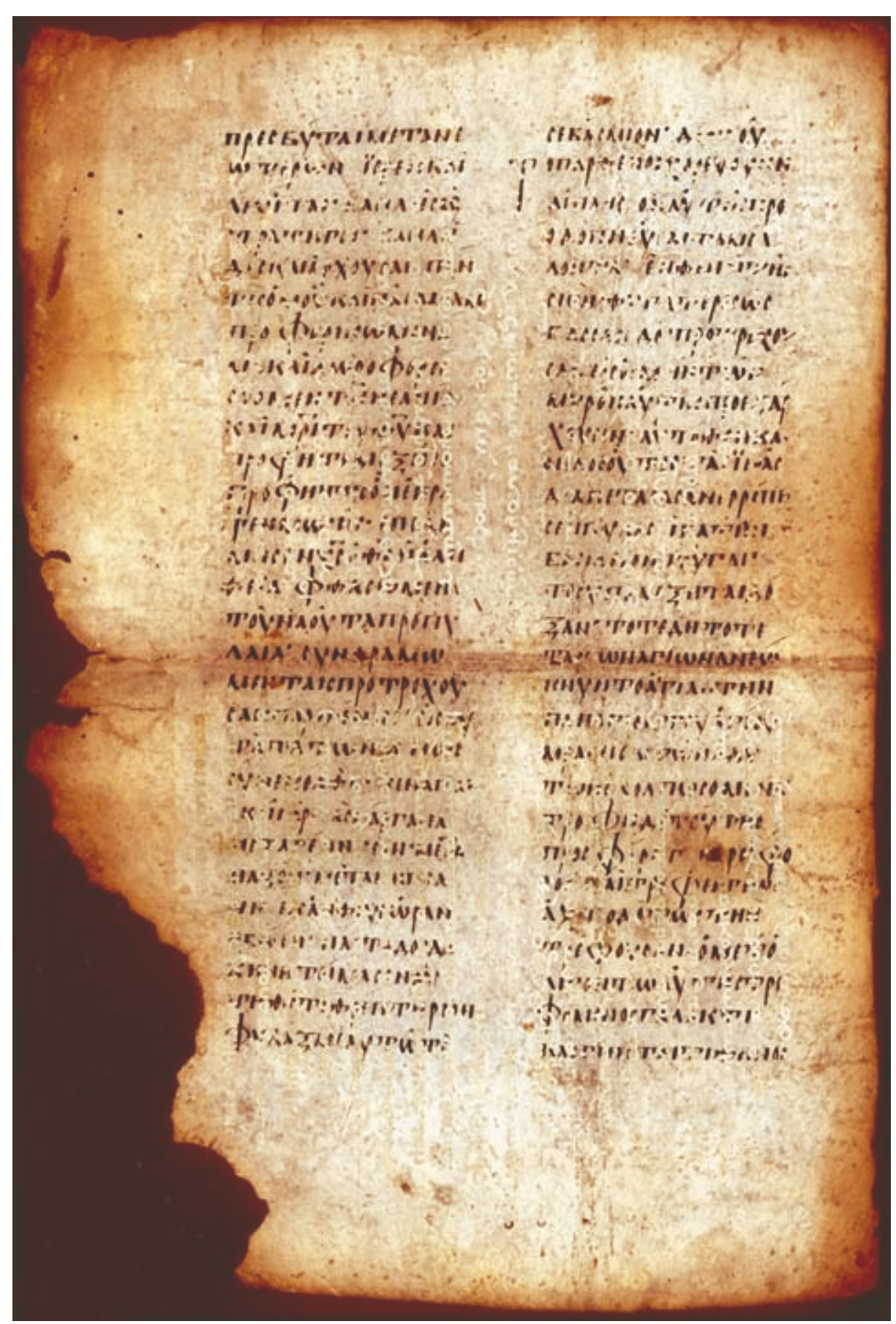

Abb. 4: Cod. Vind. theol. gr. 160, f. $214^{\mathrm{v}}+215^{\mathrm{r}}\left(=\right.$ Fragment $\mathrm{B}^{\mathrm{v}}$ ) (ca. 50\% der natürlichen Größe) (digitale Palimpsestphotographie) Copyright ( O) Österreichische Nationalbibliothek 


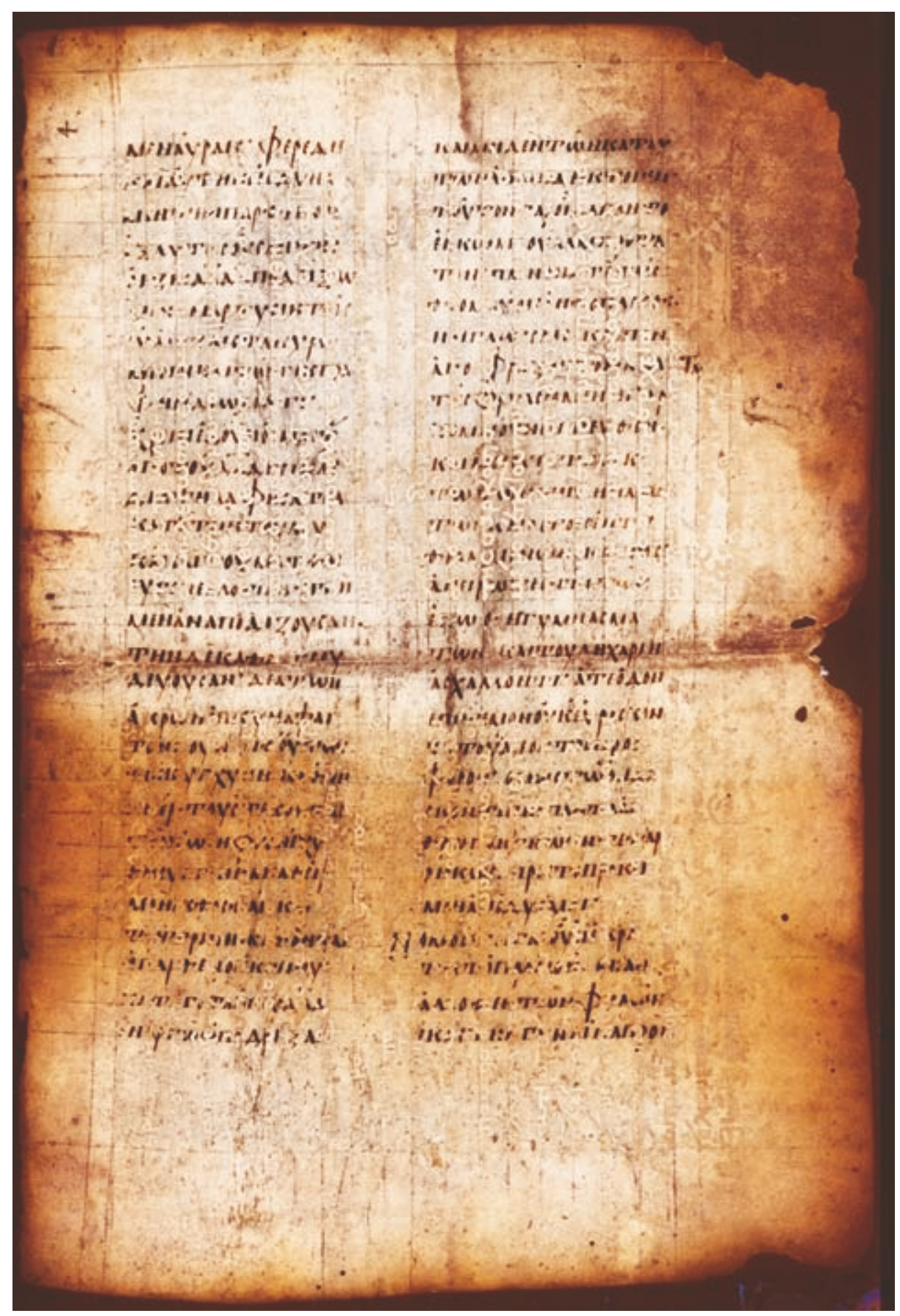

Abb. 5: Cod. Vind. theol. gr. 160, f. $4^{\mathrm{r}+1^{\mathrm{v}}}$ (= Fragment $C^{\mathrm{r}}$ ) (ca. 50\% der natürlichen Größe) (digitale Palimpsestphotographie) Copyright (C) Österreichische Nationalbibliothek 


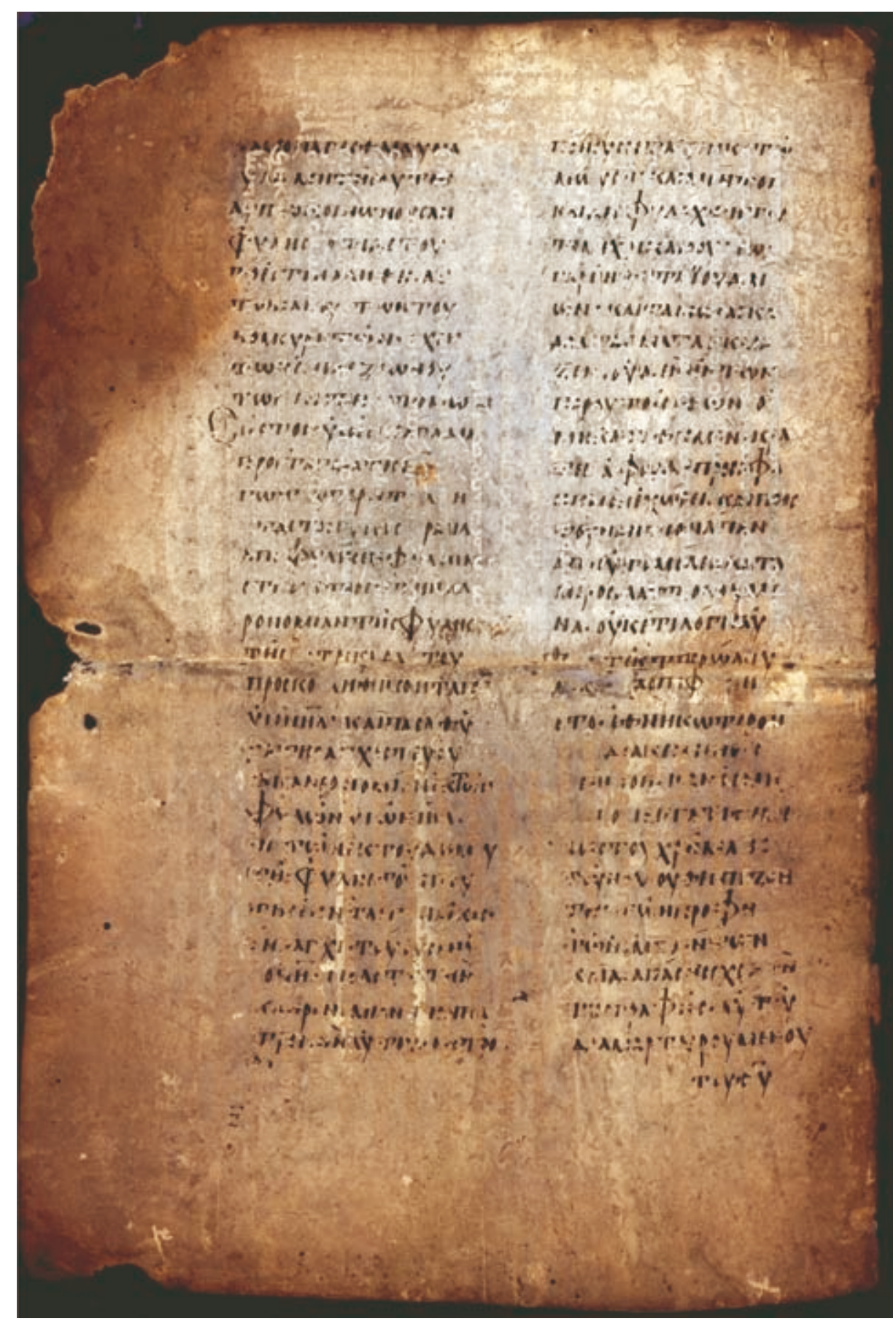

Abb. 6: Cod. Vind. theol. gr. 160, f. $4^{\mathrm{v}}+1^{\mathrm{r}}\left(=\right.$ Fragment $\left.\mathrm{C}^{\mathrm{v}}\right)$ (ca. 50\% der natürlichen Größe) (digitale Palimpsestphotographie) Copyright (C) Österreichische Nationalbibliothek 


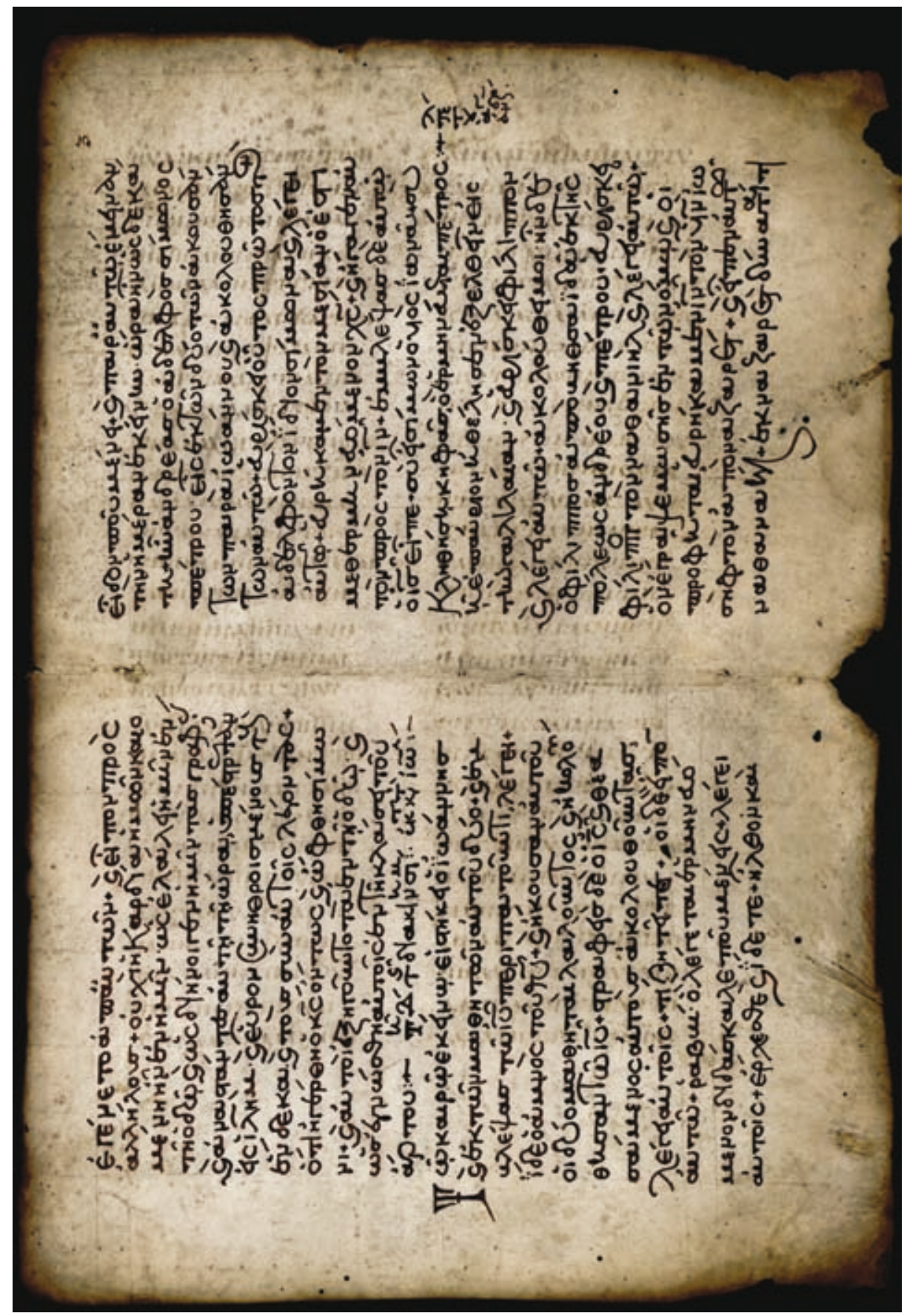

Abb. 7: Cod. Vind. theol. gr. 160, f. $3^{r}+2^{v}\left(A^{r}\right)$ : obere Schrift (ca. 50\% der natürlichen Größe) (digitale Photographie) Copyright ( $)$ Österreichische Nationalbibliothek 


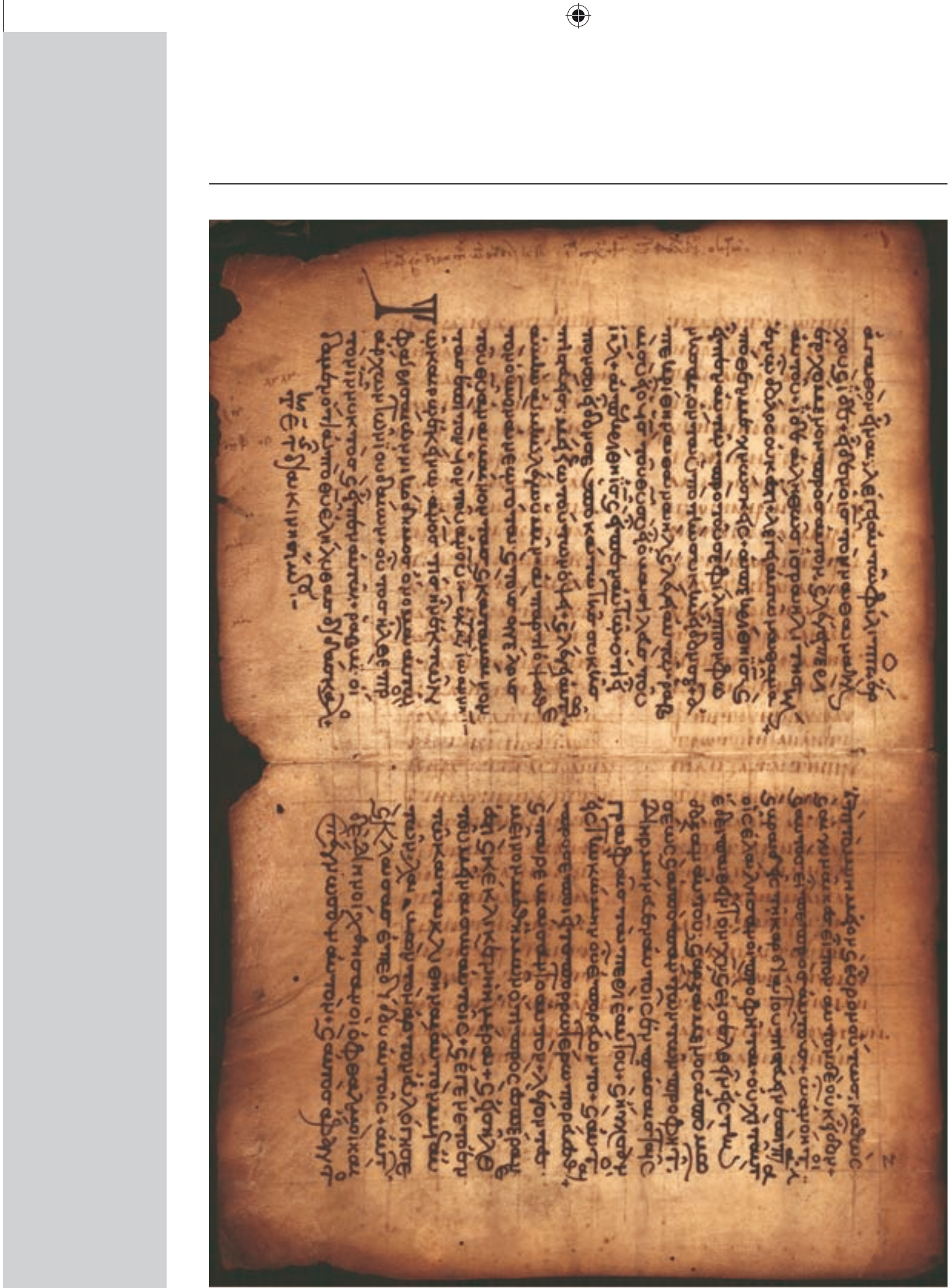

Abb. 8: Cod. Vind. theol. gr. 160, f. $3^{\mathrm{v}}+2^{\mathrm{r}}\left(\mathrm{A}^{\mathrm{v}}\right)$ : obere Schrift (ca. 50\% der natürlichen Größe) (digitale Photographie) Copyright (C) Österreichische Nationalbibliothek 

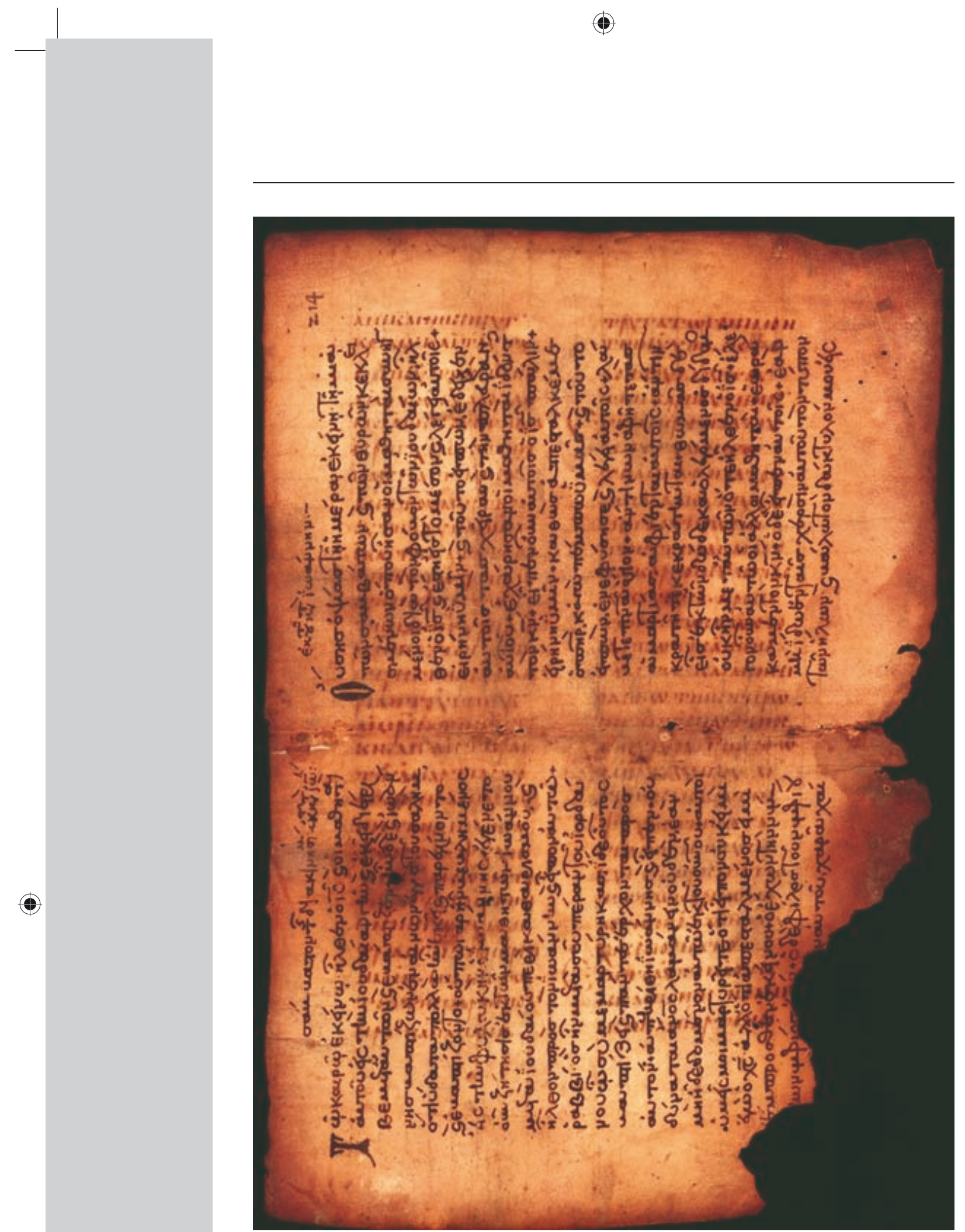

Abb. 9: Cod. Vind. theol. gr. 160, f. $214^{r}+215^{v}\left(B^{r}\right)$ : obere Schrift (ca. 50\% der natürlichen Größe) (digitale Photographie) Copyright (C) Österreichische Nationalbibliothek 


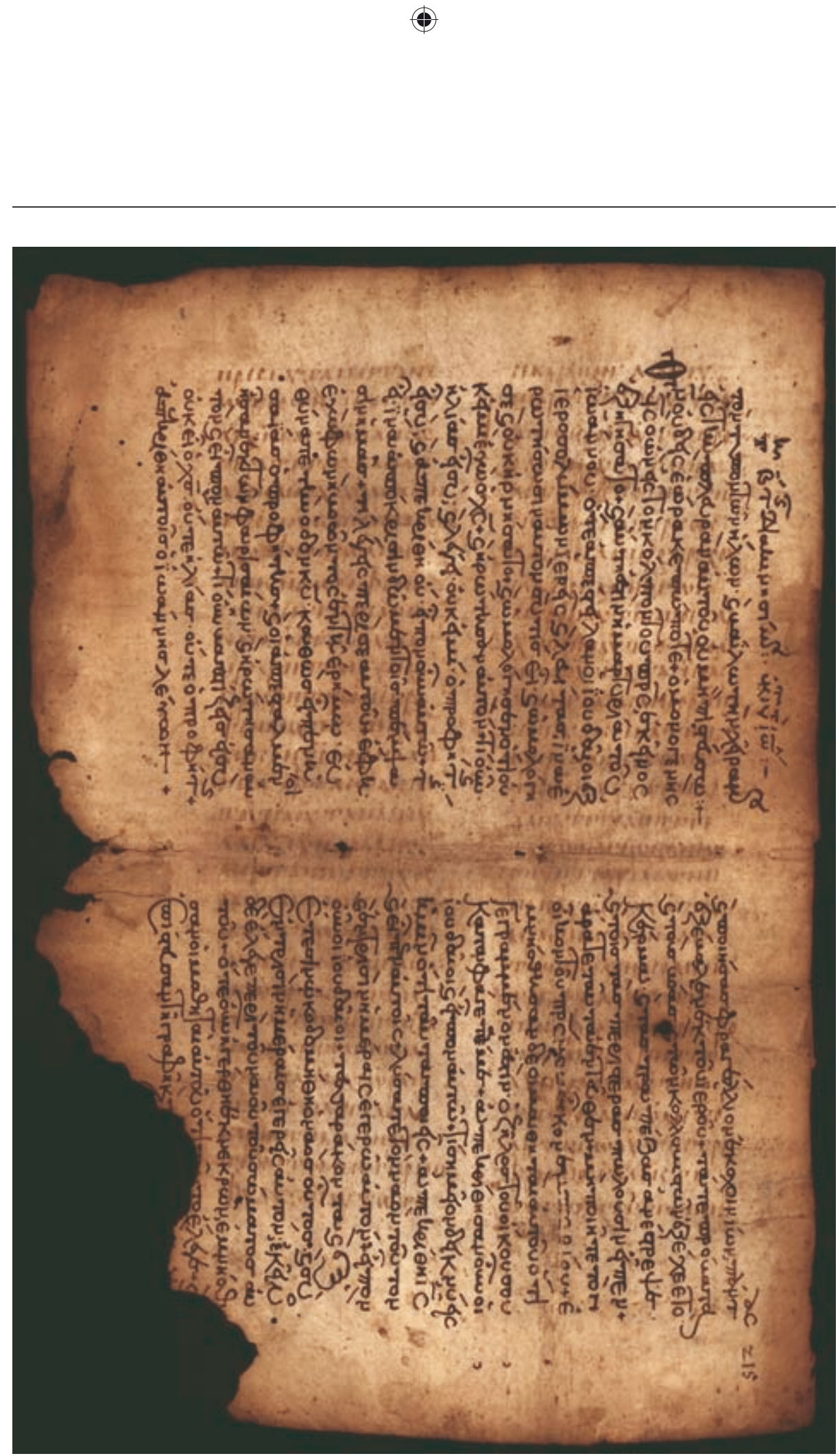

Abb. 10: Cod. Vind. theol. gr. 160, f. $214^{\mathrm{v}}+215^{\mathrm{r}}\left(\mathrm{B}^{\mathrm{v}}\right)$ : obere Schrift (ca. 50\% der natürlichen Größe) (digitale Photographie) Copyright ( O) Österreichische Nationalbibliothek 

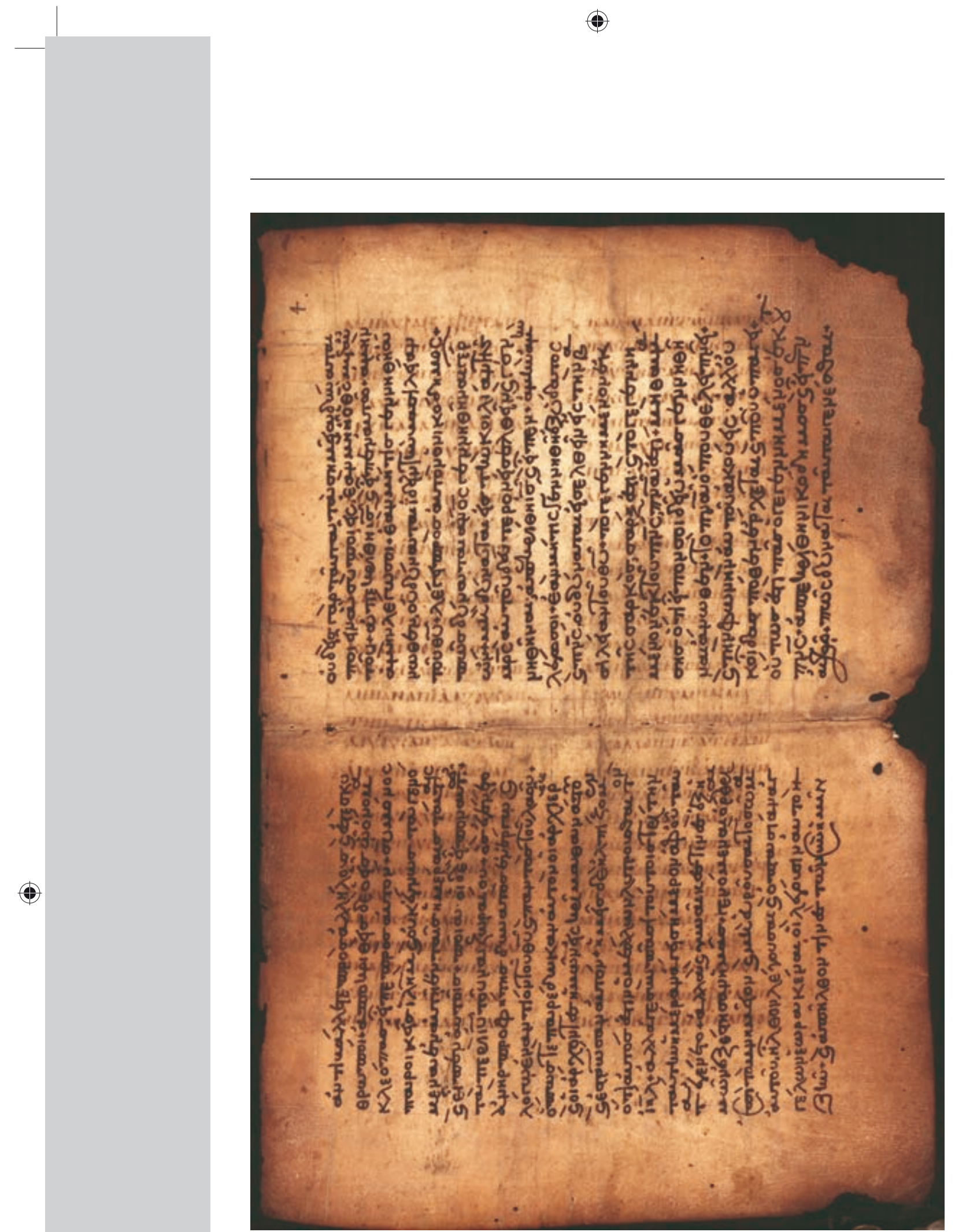

Abb. 11: Cod. Vind. theol. gr. 160, f. $4^{r}+1^{v}\left(C^{r}\right)$ : obere Schrift (ca. 50\% der natürlichen Größe) (digitale Photographie) Copyright (C) Österreichische Nationalbibliothek 


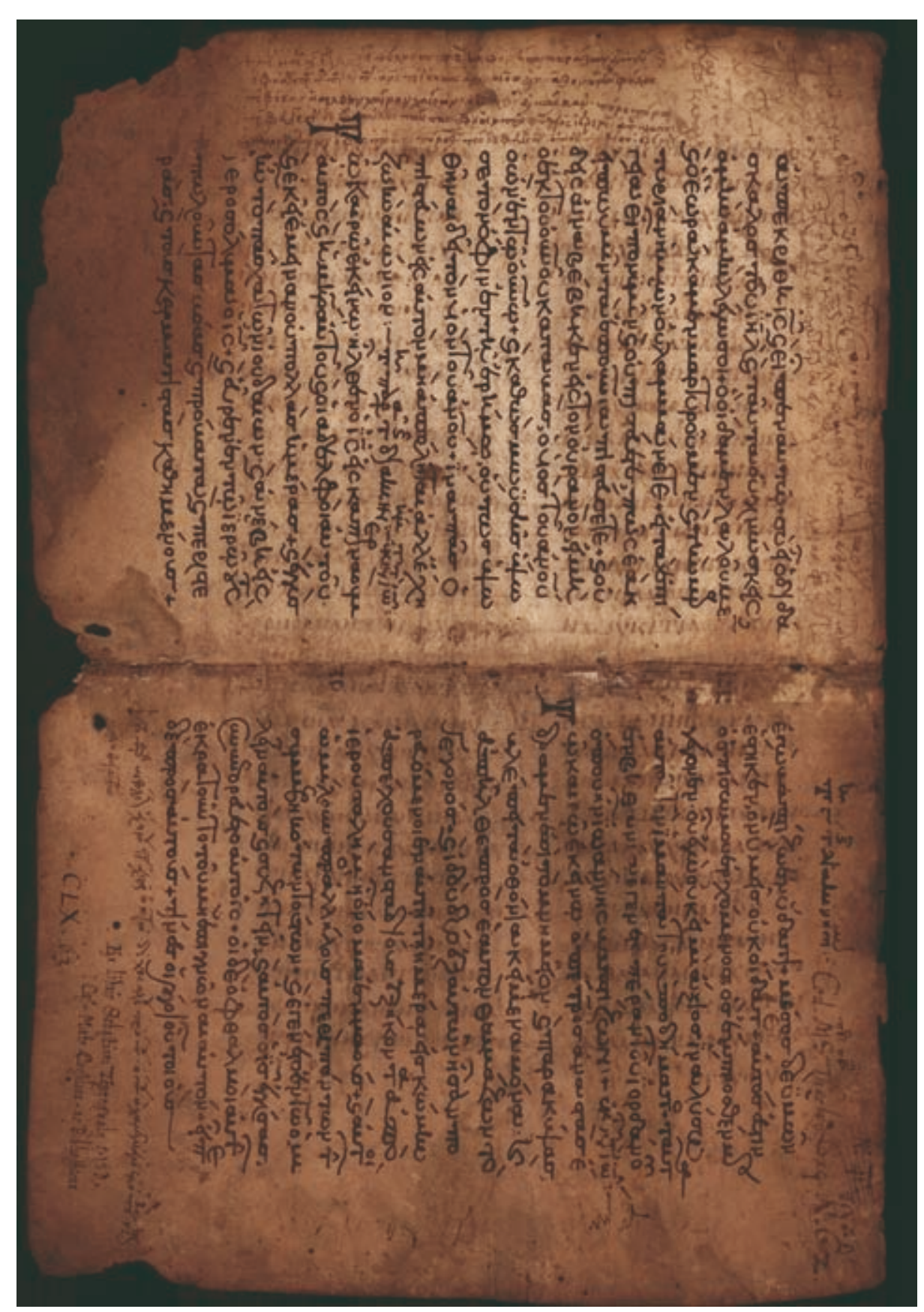

Abb. 12: Cod. Vind. theol. gr. 160, f. $4^{\mathrm{v}}+1^{\mathrm{r}}\left(\mathrm{C}^{\mathrm{v}}\right)$ : obere Schrift (ca. 50\% der natürlichen Größe) (digitale Photographie) Copyright $@$ Österreichische Nationalbibliothek 


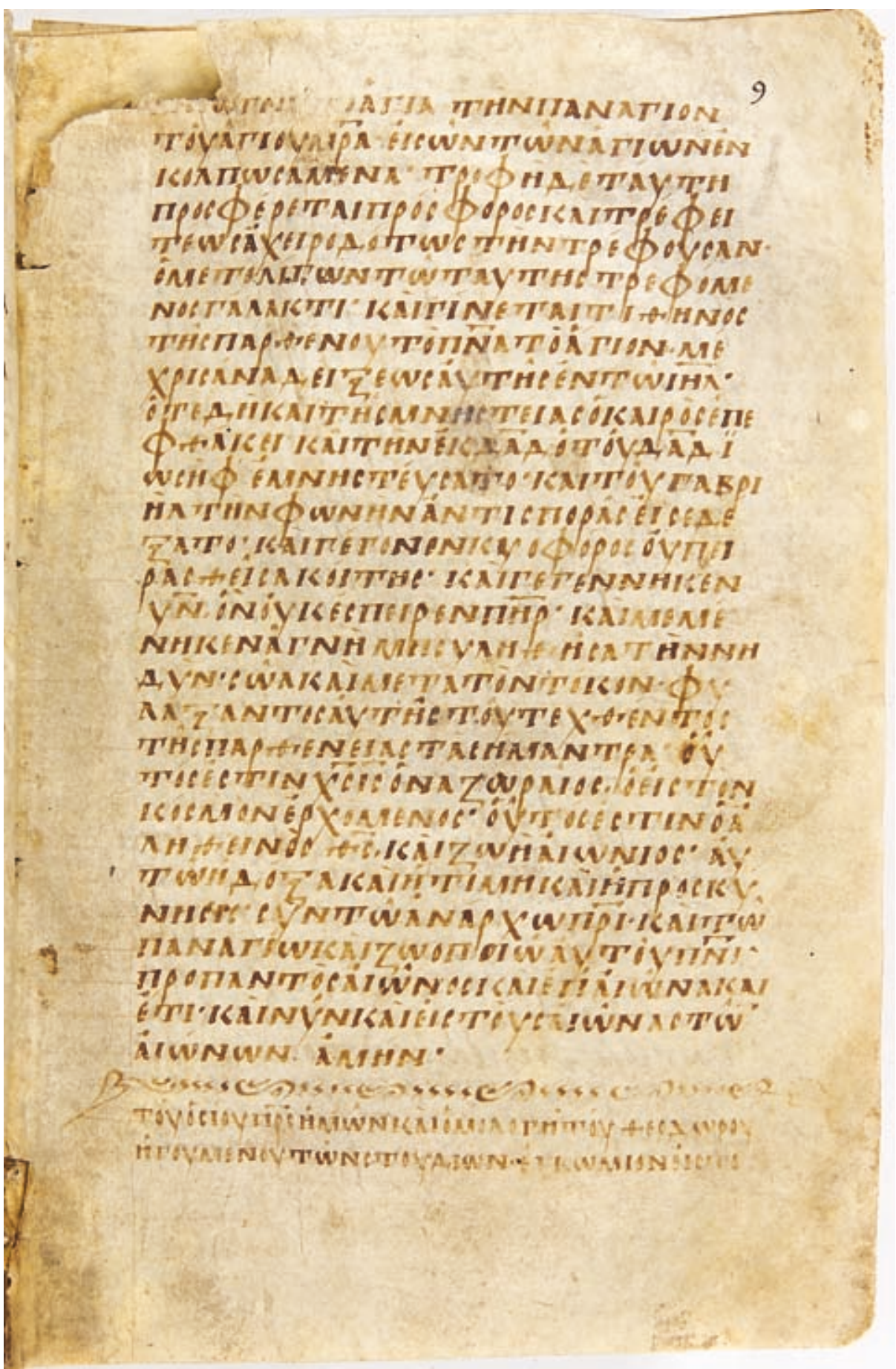

Abb. 13: Cod. Esc. $\Phi-I I I-20$ (gr. 239), f. $9^{r}$

(ca. 90\% der natürlichen Größe)

Copyright (C Patrimonio Nacional 


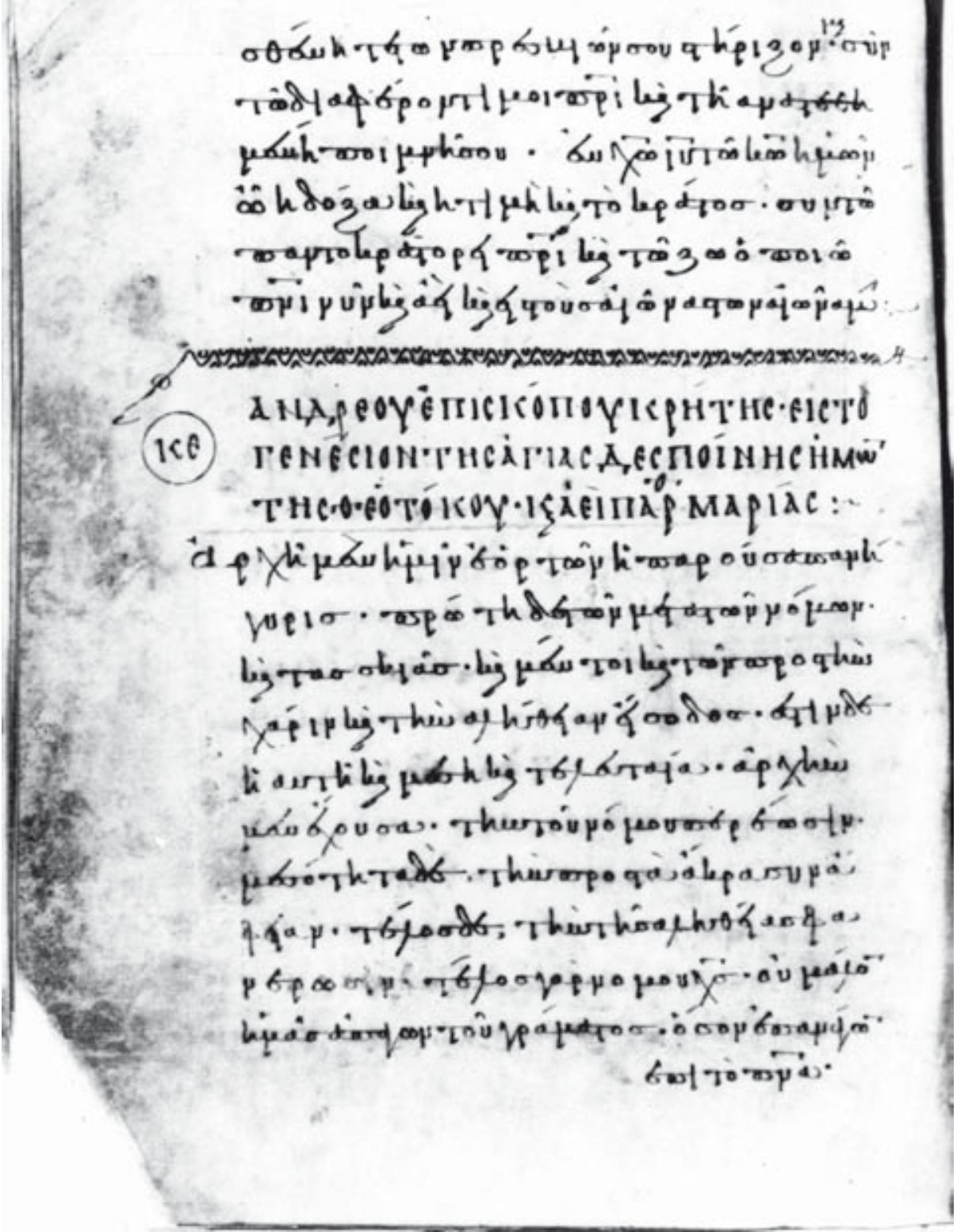

Abb. 14: Cod. Vat. gr. 2079, f. $109^{v}$

(82\% der natürlichen Größe)

Copyright $@$ Biblioteca Apostolica Vaticana 


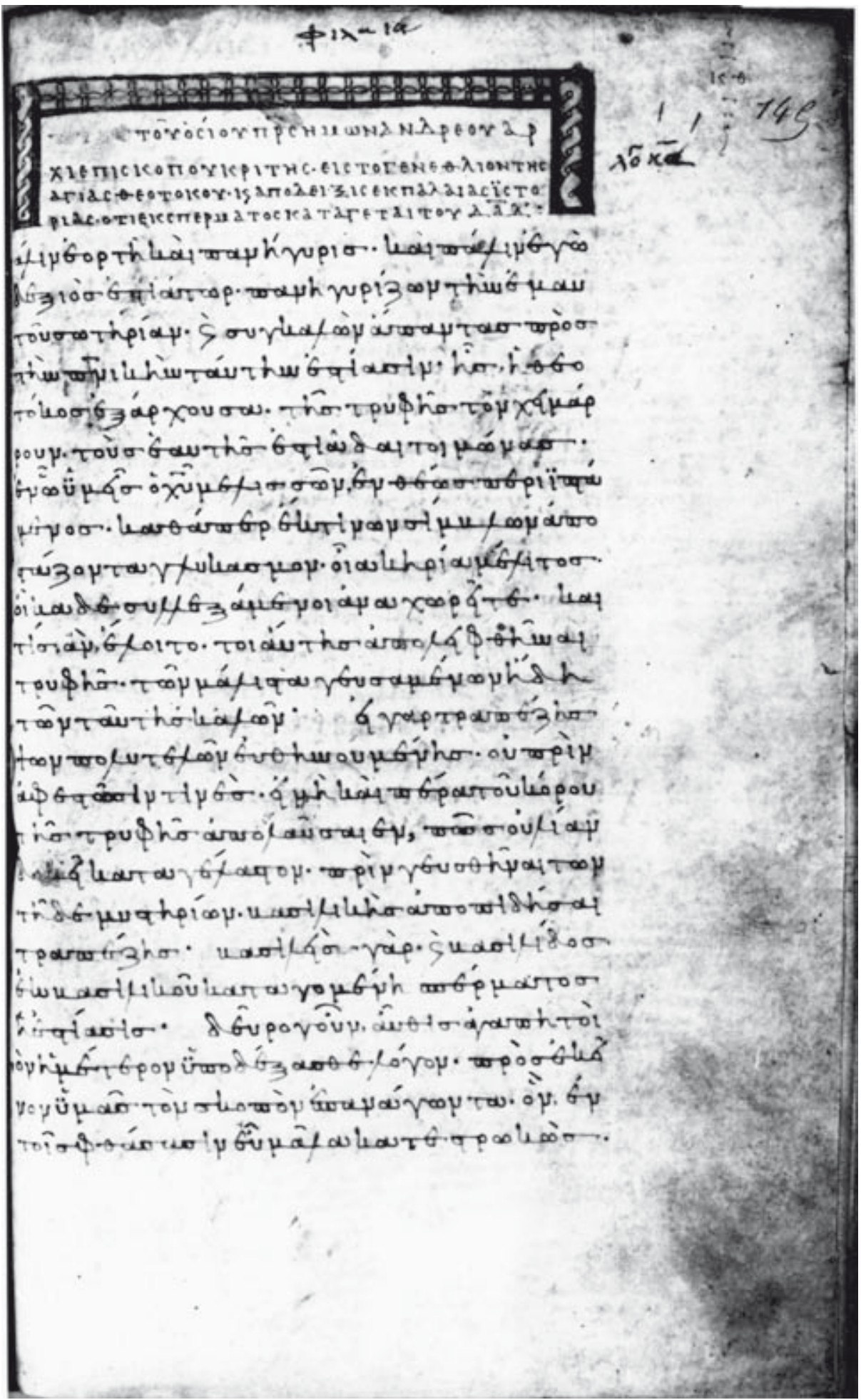

Abb. 15: Cod. Par. gr. 766, f. 145

(68\% der natürlichen Größe)

Copyright $@$ Bibliothèque nationale de France 


\section{SONDERTAFELTEIL I : INTERPUNKTION}

\begin{tabular}{|c|c|}
\hline \multicolumn{2}{|l|}{ Punkt auf der Grundlinie } \\
\hline 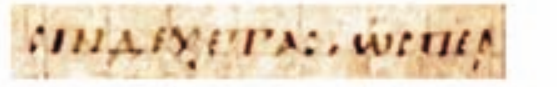 & 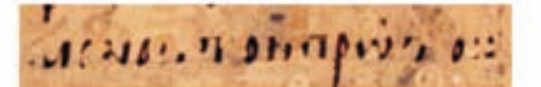 \\
\hline $\mathrm{A}^{\mathrm{v}} \Pi^{1}$ & $\mathrm{~A}^{\mathrm{v}} \mathrm{II}^{24}$ \\
\hline 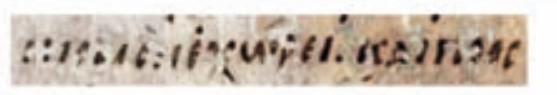 & 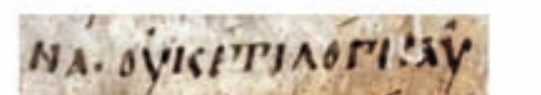 \\
\hline $\mathrm{C}^{\mathrm{v}} \mathrm{II}^{12}$ & $\mathrm{C}^{\mathrm{v}} \mathrm{II}^{16}$ \\
\hline \multicolumn{2}{|l|}{ Mittlerer Punkt } \\
\hline \multicolumn{2}{|l|}{ 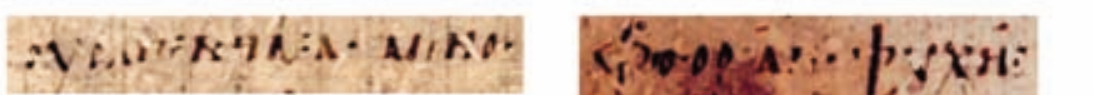 } \\
\hline$A^{v} I^{11}$ & $\mathrm{~B}^{\mathrm{r}} \mathrm{I}^{22}$ \\
\hline 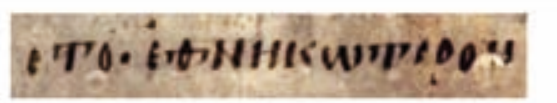 & 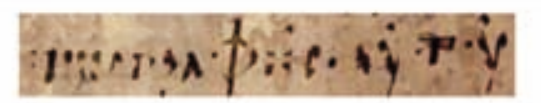 \\
\hline $\mathrm{C}^{v} \mathrm{II}^{19}$ & $\mathrm{C}^{\mathrm{v}} \mathrm{II}^{28}$ \\
\hline \multicolumn{2}{|l|}{ Hochgestellter Punkt } \\
\hline 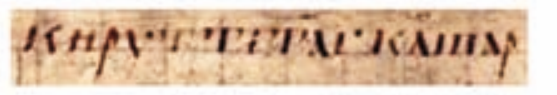 & 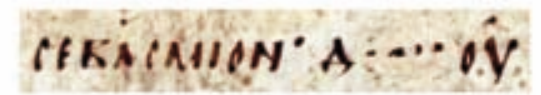 \\
\hline $\mathrm{A}^{\mathrm{V}} \mathrm{I}^{18}$ & $B^{v} I^{1}$ \\
\hline ЗAN'TROTEAHTOTIE & 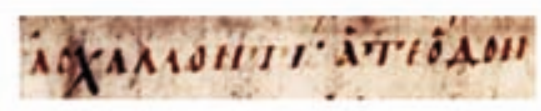 \\
\hline$B^{v} I^{16}$ & $\mathrm{C}^{\mathrm{r}} \mathrm{II}^{18}$ \\
\hline
\end{tabular}

SONDERTAFELTEIL II : NOMINA SACRA

\begin{tabular}{|c|c|c|c|c|c|}
\hline$\Delta \alpha v^{\top} \delta$ & $\Delta \alpha$ vî́ & అвós & 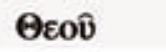 & అвôิ & $\theta \varepsilon \hat{\varphi}$ \\
\hline 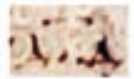 & $\alpha^{\prime} i, 3$ & 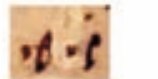 & $x \cdot \vec{y}$ & $6 y$ & ic \\
\hline $\mathrm{C}^{r} \mathrm{I}^{11}$ & $\mathrm{C}^{t} \mathrm{I}^{28}$ & $\mathrm{~A}^{\mathrm{y}} \mathrm{I}^{21}$ & $B^{v} I^{10}$ & $\mathrm{C}^{\mathrm{v}} \mathrm{II}^{30}$ & $\mathrm{~B}^{r} \mathrm{II}^{24}$ \\
\hline 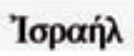 & 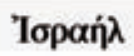 & Kúpıos & $\mu$ п்́тाр & $\mu \eta т \dot{\varepsilon} \rho \varepsilon \varsigma$ & \\
\hline IHA & 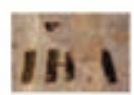 & 18 & min & Mpite. & \\
\hline $\mathrm{C}^{\mathrm{v}} \mathrm{I}^{19}$ & $\mathrm{C}^{\mathrm{v}} \mathrm{I}^{22}$ & $\mathrm{C}^{\mathrm{v}} \mathrm{II}^{1}$ & $\mathrm{~B}^{\mathrm{r}} \mathrm{II}^{19}$ & $\mathrm{~B}^{\mathrm{r}} \mathrm{I}^{17}$ & \\
\hline$\pi \alpha \tau \dot{\rho} \rho$ & $\pi \alpha \tau \dot{\varepsilon} \rho \alpha$ & $\pi v \varepsilon \dot{\mu} \mu \alpha \pi$ & $\sigma \omega \tau \eta \rho i \alpha \zeta$ & Xрıбто́ & \\
\hline ifsip & $\therefore p=$ & 171 & (सili? & $\because i$ & \\
\hline$B^{t} I^{26}$ & $\mathrm{~B}^{t} \mathrm{I}^{29}$ & $\mathrm{C}^{\mathrm{r}} \mathrm{II}^{22}$ & $\mathrm{~A}^{\mathrm{v}} \mathrm{II}^{4}$ & $B^{v} I^{14}$ & \\
\hline
\end{tabular}


